\title{
Sequence Modeling with Linear Complexity
}

by

\author{
Vasileios Lioutas \\ A Thesis submitted to \\ the Faculty of Graduate Studies and Research \\ in partial fulfilment of the requirements for the degree of
}

Master of Computer Science with Data Science Specialization

Ottawa-Carleton Institute for Computer Science

School of Computer Science

Carleton University

Ottawa, Ontario

(c) Copyright

Vasileios Lioutas, 2020 
The undersigned recommend to the Faculty of Graduate Studies and Research acceptance of the Thesis

\title{
Sequence Modeling with Linear Complexity
}

\author{
Submitted by Vasileios Lioutas \\ in partial fulfilment of the requirements for the degree of \\ Master of Computer Science with Data Science Specialization
}

Dr. Yuhong Guo, Supervisor

$\overline{\text { Dr. Matthew HolDEN, Internal Examiner }}$

Dr. Diana InkPen, External Examiner

Dr. Patrick Morin, Defence Chair

Carleton University

2020 


\section{Abstract}

Sequence modeling is one of the fundamental tasks in machine learning. As such, it has been studied for years and various different approaches have been proposed. Today, non-autoregressive attention-based methods are deemed standard for modeling sequences. Methods like Transformer which use self-attention have been shown to be capable of producing state-of-the-art results. The major drawback of self-attention is that it requires a quadratic time complexity $O\left(n^{2}\right)$ for the processing of a sequence. This makes the use of self-attention expensive and slow when it comes to long sequences. In this thesis, we aim to reduce this complexity to linear time without using attention. To do so, we introduce Time-aware Large Kernel (TaLK) Convolutions, a novel adaptive convolution operation that learns to predict the size of a summation kernel instead of using a fixed-sized kernel matrix. This method has a time complexity of $O(n)$. We evaluated our method using standard benchmark datasets in four different NLP tasks: machine translation, language modeling, abstractive text summarization and text classification. We show that our proposed adaptive convolution method is capable of achieving state-of-the-art results without using attention and with a significantly faster execution time and smaller memory footprint. 
To Vasia, my partner in crime (pt 2). 


\section{Acknowledgments}

I would like to express my gratitude to my supervisor, Dr. Yuhong Guo, who guided me throughout this project. Her support and advice were essential for the completion of this thesis. I would also like to thank my colleague Andriy Drozduyk for his feedback. Moreover, I would like to thank my beloved partner Vasileia Karasavva who supported me and encouraged me through the whole process and for reading

earlier versions of this thesis and helping me with editing. Lastly, I would like to thank my family for their support during all these years. 


\section{Table of Contents}

Abstract

\begin{tabular}{|l|l}
\hline Acknowledgments & v
\end{tabular}

Table of Contents vi vi v v v

List of Tables $\quad \mathrm{x}$

List of Figures $\quad$ xii

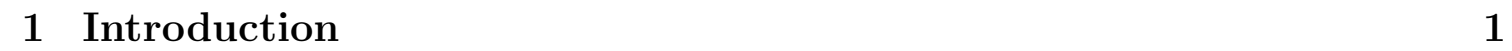

1.1 Motivation . . . . . . . . . . . . . . . . . . . . . . . 1

1.2 Contribution . . . . . . . . . . . . . . . . . . . . . . . 3

1.3 Thesis Structure . . . . . . . . . . . . . . . . . . . . 4

\begin{tabular}{lll}
\hline 2 & Background & 5
\end{tabular}

2.1 Introduction . . . . . . . . . . . . . . . . . . . . 5

2.2 Neural Networks and Deep Learning . . . . . . . . . . . . . . . 5

$2.2 .1 \quad$ Feed-forward Neural Networks . . . . . . . . . . . . . . . . . . 6

2.2 .2 Back-Propagation . . . . . . . . . . . . . . . 8

2.3 Convolution Neural Networks $\ldots \ldots \ldots$. . . . . . . . . . . . . . 9

2.3 .1 Depthwise Convolutions . . . . . . . . . . . . . . . . . . 10

2.3 .2 Dilated Convolutions . . . . . . . . . . . . . . . . . . . . . . . 12 
2.4 Recurrent Neural Networks. . . . . . . . . . . . . . . . . . . . . . . . . . $\quad 13$

2.4 .1 Gated RNNs . . . . . . . . . . . . . . . . . . . . 14

2.4 .2 Bidirectional RNN $\ldots \ldots \ldots \ldots \ldots$

2.5 Attention $\ldots \ldots \ldots \ldots \ldots \ldots \ldots$

$2.6 \quad$ Word Embedding and Subword Tokenization . . . . . . . . . . . . . 19

2.7 Text Generation . . . . . . . . . . . . . . . . . . . . . . . . . . . . . . . 21

2.7 .1 Conditioned Text Generation . . . . . . . . . . . . 21

2.8 Beam Search $\ldots \ldots \ldots \ldots$

2.9 Evaluation Metrics $\ldots \ldots \ldots \ldots \ldots$

2.9 .1 BLEU- $n$ Score . . . . . . . . . . . . . . . . . . . . 24

2.9 .2 ROUGE Score. . . . . . . . . . . . . . . . . . . . 25

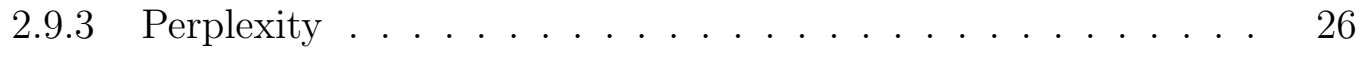

2.9 .4 Classification Accuracy . . . . . . . . . . . . . . 27

\begin{tabular}{|lll}
\hline 3 & Related Work & 28 \\
\hline
\end{tabular}

3.1 Introduction $\ldots \ldots \ldots \ldots \ldots \ldots \ldots$

3.2 Sequence Modeling . . . . . . . . . . . . . . . . . . . . . 28

3.2 .1 Recurrent-based Methods . . . . . . . . . . . . . . . . . . . 29

3.2 .2 Convolution-based Methods . . . . . . . . . . . . . 32

3.2 .3 Attention-based Methods. . . . . . . . . . . . . . . . . 37

3.3 Dynamically Sized Receptive Field $\ldots \ldots \ldots$. . . . . . . . . 42

$3.3 .1 \quad$ Deep Neural Networks with Box Convolutions . . . . . . . . . 42

3.3 .2 Large-Kernel Convolution Using Summed-Area Tables . . . . 43

\begin{tabular}{|lll}
4 & Proposed Method & 44
\end{tabular}

4.1 Introduction . . . . . . . . . . . . . . . . . . . . . . . . . . 44

4.2 Motivation . . . . . . . . . . . . . . . . . . . . . 44

4.3 One-dimensional Large Kernel Convolution . . . . . . . . . . . . . 45 
4.3 .1 Summed-area Table . . . . . . . . . . . . . . . . . . . . . 46

4.4 Time-aware Large Kernel Generation . . . . . . . . . . . . . . . 47

4.5 Output Normalization and Offsets Dropout . . . . . . . . . . . . 49

4.6 Multi-headed Kernels . . . . . . . . . . . . . . . . . . . . . . . . . . . 50

4.7 Decoding Using TaLK Convolutions . . . . . . . . . . . . . . . . 50

4.8 Module Architecture and Implementation. . . . . . . . . . . . 52

4.9 Computational Complexity . . . . . . . . . . . . . . . . . . . . 54

4.10 Conclusion $\ldots \ldots \ldots \ldots \ldots \ldots \ldots$

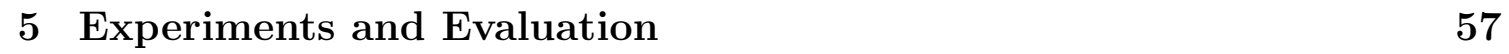

5.1 Introduction . . . . . . . . . . . . . . . . 57

5.2 Machine Translation . . . . . . . . . . . . . . . . 57

5.2 .1 Datasets . . . . . . . . . . . . . . . . . 58

$5.2 .2 \quad$ Experiment Details $\ldots \ldots \ldots \ldots \ldots$

5.2 .3 Results . . . . . . . . . . . . . . . . . . . . . . . 64

5.3 Language Modeling . . . . . . . . . . . . . . . . . . . . 66

5.3 .1 Datasets . . . . . . . . . . . . . . . . . 67

$5.3 .2 \quad$ Experiment Details . . . . . . . . . . . . . . . . 68

5.3 .3 Results . . . . . . . . . . . . . . . . . . . . 70

5.4 Abstractive Text Summarization. . . . . . . . . . . . . . . 71

5.4 .1 Datasets . . . . . . . . . . . . . . . . . . . 72

5.4 .2 Experiment Details . . . . . . . . . . . . . . . . . 73

5.4 .3 Results . . . . . . . . . . . . . . . . . . . . 74

5.5 Sentence Classification $\ldots \ldots \ldots \ldots \ldots \ldots$

5.5 .1 Datasets . . . . . . . . . . . . . . . . . . 75

$5.5 .2 \quad$ Experiment Details $\ldots \ldots \ldots \ldots \ldots$

5.5 .3 Results . . . . . . . . . . . . . . . . . 77 
5.6 Ablation Study . . . . . . . . . . . . . . . . . . . . . . . . 77

5.7 Encoding Inference Speed Comparison . . . . . . . . . . . . . . . . . 79

5.8 Conclusion . . . . . . . . . . . . . . . . . . . . . . . . . . . . . . 81

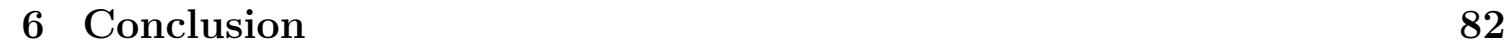

6.1 Summary and Conclusion . . . . . . . . . . . . . . . . . . . . 82

6.2 Future Work . . . . . . . . . . . . . . . . . . . . . . . 83

\begin{tabular}{lr}
\hline List of References & 84
\end{tabular}

\begin{tabular}{|lll}
\hline Appendix A TaLK Convolution CUDA Pseudocode & 91
\end{tabular}

A.1 $\quad$ Encoder-optimized Implementation . . . . . . . . . . . . . . . . . . . 91

A.1.1 Forward Implementation . . . . . . . . . . . . . . . . . . . . . 91

A.1.2 Backward Implementation . . . . . . . . . . . . . . . . . . . . 92

A.2 Decoder-optimized Implementation . . . . . . . . . . . . . . . . . . . 94

A.2.1 Forward Implementation . . . . . . . . . . . . . . . . . . . . 94

A.2.2 Backward Implementation . . . . . . . . . . . . . . . . . 95

A.3 Pytorch Function Implementation . . . . . . . . . . . . . . . . . . . . 96 


\section{List of Tables}

2.1 Common choices of non-linear functions. . . . . . . . . . . 7

$4.1 \quad$ Maximum path lengths, per-layer complexity and minimum number of sequential operations for different layer types. $n$ is the sequence length, $d$ is the representation dimension and $k$ is the kernel size of convolutions. 54

5.1 Machine translation benchmark datasets statistics. . . . . . . . . 58

$5.2 \quad$ Machine translation accuracy in terms of BLEU for WMT En-Fr on

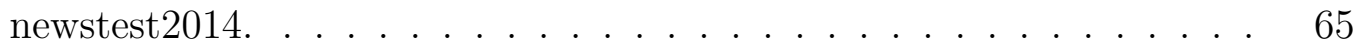

$5.3 \quad$ Machine translation accuracy in terms of BLEU for WMT En-De on newstest2014. . . . . . . . . . . . . . . . . 66

5.4 Machine translation accuracy in terms of BLEU on IWSLT De-En. . 67

$5.5 \quad$ WikiText-103 benchmark dataset for language modeling. . . . . . . . 68

5.6 Test perplexity on WikiText-103. We used adaptive inputs similar to 11 and show that our method yields better perplexity than selfattention using adaptive inputs. . . . . . . . . . . . . . . 71

5.7 CNN/DailyMail benchmark dataset for abstractive summarization. . 72

5.8 Results on CNN/DailyMail summarization. . . . . . . . . 75

5.9 Results on IMDB Movies Reviews dataset. $\ldots \ldots \ldots \ldots \ldots$

5.10 Ablation on IWSLT De-En validation set. (+) indicates that a result includes all preceding features. . . . . . . . . . . . . . . 78 
5.11 Throughput and memory consumption decrease measured for different sequence lengths $(n)$ on a batch of size 10 with each token being represented with $d=1024$ and $H=16$. Throughput is calculated across 100K iterations of a single input encoding execution for each method. Memory decrease is computed as how many times less memory we need to encoding the input embedding compared to Self-Attention. Larger numbers indicate better performance. . . . . . . . . . . . . . . . . . . 79 


\section{List of Figures}

2.1 Different level of abstraction for image and text data. . . . . . . . . . 6

2.2 A 3-layer neural network with three inputs, two hidden layers of 4

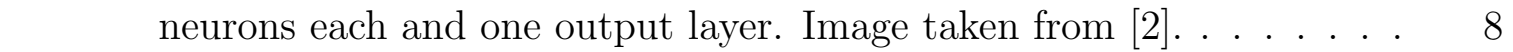

2.3 An example of convolution operation in 2D space. Image taken from [3] 9

2.4 An example of depthwise convolution operation in 2D space. Image

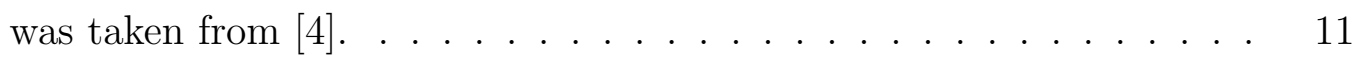

2.5 The dilated convolution operation. Subfigure (a) shows how normal convolution scans over an image. Subfigure (b) shows how dilated convolution with dilation size 2 has an effective receptive field of $7 \times 7$ while using a kernel of size $3 \times 3$. Subfigure (c) shows a dilated convolution with dilation size 4. Figures taken from [5]. . . . . . . . . . . . . . . 13

2.6 A recurrent neural network unit. The image is taken from $[6]$. . . . . 14

$2.7 \quad$ A long short-term memory unit. The figure is taken from $|7|$. . . . . 15

$2.8 \quad$ A gated recurrent neural network unit. The image is taken from $|6|$. . 17

2.9 The general framework of a language model. . . . . . . . . . . . . . . 22

3.1 The first seq2seq LSTM-based approach. [8] . . . . . . . . . . . . . 30

3.2 Attention with LSTM-based approaches. . . . . . . . . . . . . . . . 30

$3.3 \quad$ A very deep LSTM-based neural machine translation architecture proposed by He et al. $|9|$. . . . . . . . . . . . . . . . . . . . . . . 32 
3.4 The first convolution-based sequence modeling approach proposed by Kaiser et al. $|10|$.$] . . . . . . . . . . . . . . . . . . . . . . . . 33$

3.5 The first convolution-based sequence modeling approach that is based only on convolutions in a non-autoregressive way. The image was taken from $\mid 11]$.

3.6 Lightweight and Dynamic convolution units proposed by $\mathrm{Wu}$ et al. $12 \mid$. 36

3.7 The Transformer's multi-head self-attention unit. The image was taken from $[13 \mid . \ldots \ldots \ldots 37$

$3.8 \quad$ The first convolution-based sequence modeling approach proposed by

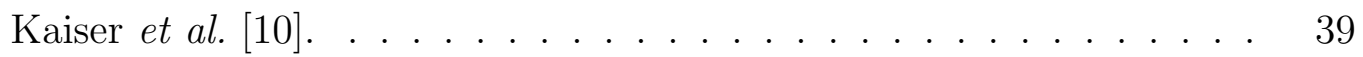

3.9 The process of Locality-sensitive-hashing that Reformer uses. Image

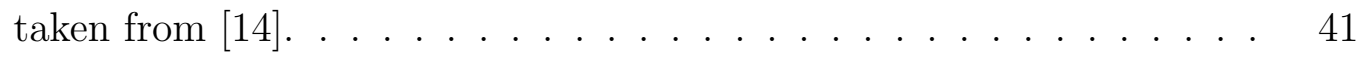

3.10 The progress of learning box coordinates of box convolutions. The figures was taken from $\mid 15] . \ldots$. . . . . . . . . . . . . . . . . . 42

3.11 The interpolation approach for using real valued box coordinates as proposed by Zhang et al. $[16]$. . . . . . . . . . . . . . . . . . . . . 43

4.1 The One-dimensional Large Kernel Convolution operation. For the current time-step, given the left and right offsets, we sum all the representation vectors inside these boundaries. . . . . . . . . . . . . . . . 47

4.2 The Time-aware Large Kernel convolution operation. For the current time-step, we compute the left and right offsets for each head, and then sum all the representation vectors inside these boundaries. This operation can be efficiently computed using summed-area tables with time complexity $O(\log (n))$ and compute the output representation for each time-step in $O(n)$ time. . . . . . . . . . . . . . . . . . 51

4.3 The architecture of the proposed TaLK Convolution unit. . . . . . . . 52

4.4 The model architecture of the proposed TaLK Convolution network. . 53 
5.1 The cosine learning rate scheduler used with WMT En-Fr and WMT En-De bechmark datasets. . . . . . . . . . . . . . . . . . . . . . . . . 62

5.2 The inverse square root learning rate scheduler used with the IWSLT De-En bechmark dataset. . . . . . . . . . . . . . . . . . . . . . . . . 63

$5.3 \quad$ Words are assigned to clusters $\mathcal{V}_{i}$ based on their frequency which determines the size of the representations. Embeddings are projected to a common dimension $d$ before being fed to the model. Figure taken from $[1] \ldots \ldots \ldots \ldots \ldots \ldots$ 


\section{Chapter 1}

\section{Introduction}

\subsection{Motivation}

Sequence modeling is a highly active research area. It is the process of predicting the next time-step input given some, or in certain cases all, of the previous time-steps. Sequence modelling has applications on many tasks including financial time series prediction, speech recognition, audio generation, sentiment classification, machine translation and video activity recognition. It is widely studied for highly unstructured data such as text sequences as this type of data introduces a challenging learning task with a plethora of data samples available.

Since the introduction of neural networks, sequence modeling has seen some great breakthroughs. More recently, there has been a lot of progress in sequence modeling through recurrent neural networks $(\mathrm{RNN})$ [8, 17, 18, RNN is a natural fit with this type of modeling since it allows to exhibit a temporal dynamic behavior. An RNN network has a time complexity of $O(n)$ where $n$ is the length of the sequence but since the method is autoregressive, it depends on the output of the previous step, which makes the algorithm not parallelizable. 
The research community has concentrated its effort to develop non-autoregressive approaches that can take advantage of the highly parallelizable hardware that exists today for the last few years. Convolutions $[11,12,19,21]$ and attention $[13,14,22,23$ played an important role over the years in order to achieve this. All current state-of-the-art methods of sequence modeling rely on the use of attention to "filter" the excessive information given at a current time-step. Attention can be expressed as the weighted sum over context representations using attention weights that are typically generated from the context representations (self-attention).

The transformer network assigns attention weights for a given time-step to all available context token representations, while the newly proposed dynamic convolution only computes an attention over a fixed context window. Self-attention over all context tokens is computationally-speaking, very expensive. More specifically, the transformer network has a time complexity of $O\left(n^{2}\right)$ where $n$ is the length of the input sequence. Thus, modeling long-range dependencies becomes very challenging and the practicality of the self-attention method has been questioned. The more recent approach of dynamic convolution successfully reduced the time complexity to $O(k \cdot n)$ where $k$ is the kernel size specified for each layer.

In this thesis, we introduce a novel type of adaptive convolution, the Time-aware Large Kernel (TaLK) convolution, that learns the kernel size of a summation kernel for each time-step instead of learning the kernel weights as in a typical convolution operation. For each time-step, a function is responsible for predicting the appropriate size of neighbor representations to use in the form of left and right offsets relative to the time-step. The result is an efficient encoding method that reduces the time complexity to $O(n)$ and uses fewer parameters than all other methods. The method employs the fast Parallel Prefix Sum operation which has a time complexity 
of $O(\log (n))$ to compute the integral image, also known as summed-area table in the Computer Vision literature. This needs to be computed only once and can be used to calculate any summation between two boundary tokens in $O(1)$. Applying it on a sequence with length $n$ only needs $O(n)$ time.

\subsection{Contribution}

The contributions of this thesis (and their respective chapters) are as follows:

- We introduce a novel adaptive convolution based on summation kernel for sequence encoding.

- We show both analytically and empirically that the proposed kernel method has a smaller time complexity; it is faster than previous state-of-the-art approaches and is able to encode longer sentences quicker and with a smaller running memory footprint.

- We evaluate our method on four core NLP tasks, machine translation, language modeling, abstractive text summarization and sequence classification using in total six different benchmark datasets. We show that the proposed method can get comparative performance with previous methods achieving state-of-the-art results. 


\subsection{Thesis Structure}

The rest of the thesis is organized as follows.

Chapter 2: In this chapter, we introduce the basic concepts of machine learning and deep learning that are necessary for the understanding of the proposed sequence modeling approach. Readers familiar with deep learning and natural language processing may skip this chapter.

Chapter 3: In this chapter, we go over some of the most important works in the literature that have shaped the state-of-the-art sequence modeling methods. We give an overview of the recurrent-based, convolution-based and attention-based methods. In addition, we discuss several methods for adaptively enlarging the receptive field of the convolution operation.

Chapter 4: In this chapter, we introduce our proposed Time-aware Large Kernel (TaLK) convolution method. We explain the motivation behind it and we show how to create an adaptive version for each input sequence. We present the proposed architecture and compare our method's computational time complexity against other state-of-the-art methods from the empirical literature.

Chapter 5: In this chapter, we present our experimental findings that assert that our method is capable of yielding state-of-the-art results with faster execution time. We evaluate our method in four natural language processing tasks: neural machine translation, language modeling, abstractive summarization and sentence classification.

Chapter 6: In this chapter, we present our conclusion and make suggestions for future research in related areas. 


\section{Chapter 2}

\section{Background}

\section{$2.1 \quad$ Introduction}

In this chapter, first we describe the general framework of deep learning and how to train neural networks through gradient descent (Section 2.2). Based on this, we continue by introducing the notion of convolutions (Section 2.3) and recurrent neural networks (Section 2.4). We discuss about the attention mechanism and give the formal definition of the procedure (Section 2.5). We explain how we can represent words in deep learning (Section 2.6) and formalize how to learn to generate text using neural networks (Section 2.7). Finally, we describe the inference searching algorithm used with sequence generation models (Section 2.8) and define the metrics (Section 2.9 that our proposed sequence modeling method will be evaluated on.

\subsection{Neural Networks and Deep Learning}

Deep learning attempts to extract the underlying factors of variation of the data in a hierarchical manner. For example, an image can be described as a set of pixels, edges or objects and similarly a text sentence can be broken down to a set of words, entities and high-level meanings (Figure 2.1). A deep learning model primarily 


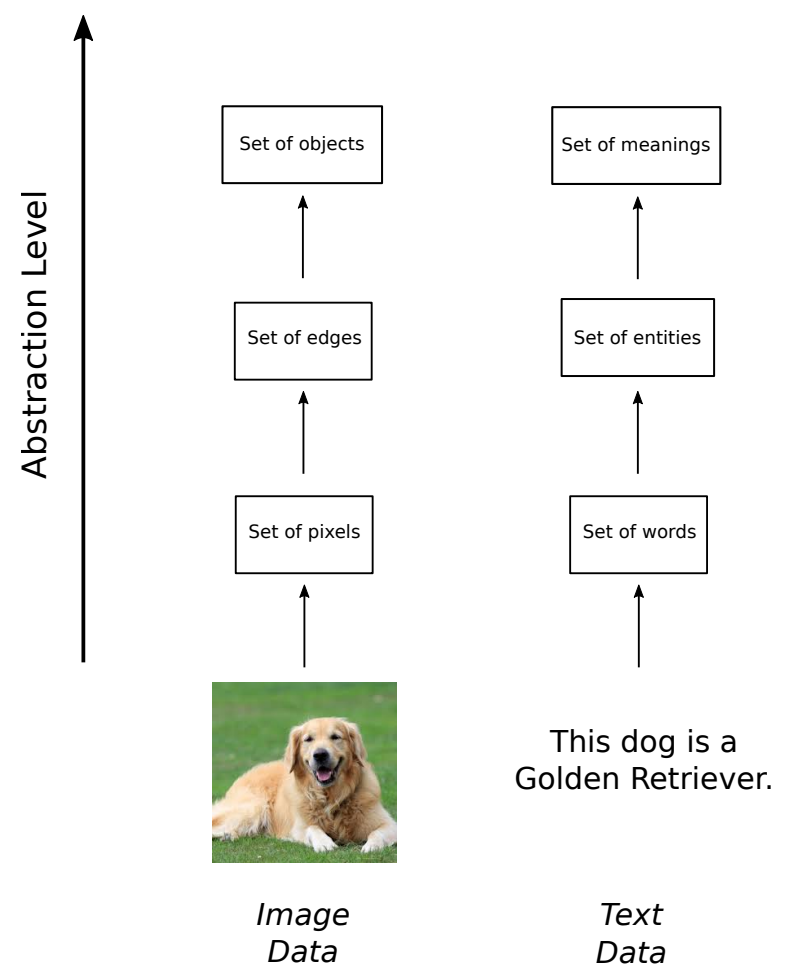

Figure 2.1: Different level of abstraction for image and text data.

consists of multiple layers (hierarchies) of feed-forward neural networks where each layer is responsible to learn either implicitly or explicitly representations of the aforementioned abstractions. This is done through a trial-and-error procedure where the learning model is forced to take a (large) number of input examples and predict the desired output enforced by using an objective (loss) function.

Deep learning approaches are particularly popular due to the good performance they yield. This is due to the great amount of data available for training as well as the highly parallelizable and optimized hardware (e.g. GPU and TPU) that exists.

\subsubsection{Feed-forward Neural Networks}

A feed-forward neural network is the building block of every deep learning model. 
Definition 2.2.1. (Feed-forward Neural Network). Given a matrix $W \in \mathbb{R}^{d \times k}$ and a vector $b \in \mathbb{R}^{k}$, a feed-forward neural network is defined as $f(x)=g\left(W^{T} x+b\right)$, where $x \in \mathbb{R}^{d}$ is the input representation vector and $g(\cdot)$ is a non-linear differentiable function. The $W$ and $b$ are called learnable parameters and often both are referred as $\theta$ denoting all parameters of the network. These $\theta$ parameters are learned through gradient-based optimization methods.

Over the years, researchers in the community proposed many different non-linear functions $g(\cdot)$, each with its own intuition on why they help the optimization process of a neural model. Table 2.1 shows some of the common choices used in the literature.

Table 2.1: Common choices of non-linear functions.

\begin{tabular}{ll}
\hline Name & Formula \\
\hline sigmoid $(\sigma)$ & $g(x)=\frac{1}{1+e^{-x}}$ \\
tanh & $g(x)=\frac{e^{x}-e^{-x}}{e^{x}+e^{-x}}$ \\
ReLU & $g(x)=\max (0, x)$ \\
Leaky ReLU & $g(x)=\mathbb{1}(x<0)(\alpha x)+\mathbb{1}(x>=0)(x)$ \\
\hline
\end{tabular}

A deep neural network (DNN) is defined as a graph composed of multiple stacked feed-forward layers (Figure 2.2), where the output of the $i$-layer is the input to the $(i+1)$-layer. The depth of a neural network is defined as the number of its layers.

We denote as $\hat{y}=f(x ; \theta)$ the output of the deep neural network. In supervised learning, we typically train the network using maximum likelihood as the objective function. Thus, we compute the negative log-likelihood given by

$$
J(\theta)=-\mathbb{E}_{x, y \sim \hat{p}_{\text {data }}} \log p_{\text {model }}(y \mid x)
$$




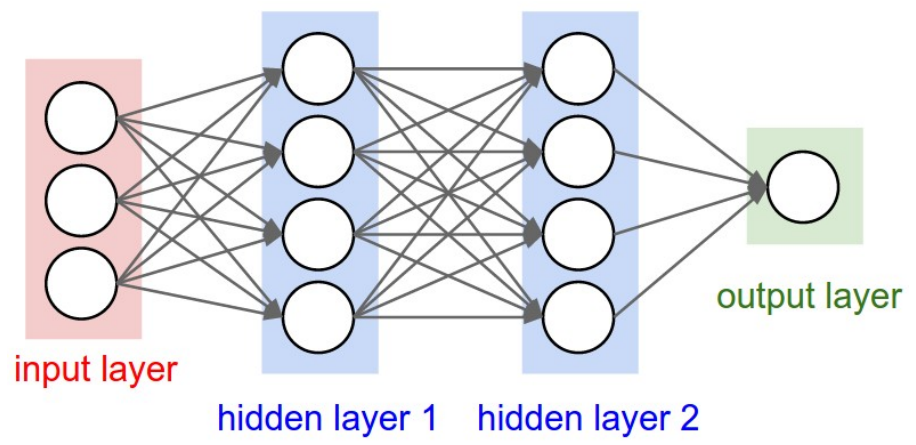

Figure 2.2: A 3-layer neural network with three inputs, two hidden layers of 4 neurons each and one output layer. Image taken from [2].

This process is called forward propagation.

\subsubsection{Back-Propagation}

The back-propagation algorithm (Algorithm 1) allows the information from the loss value that is computed using the Equation 2.1, to "flow" backwards through the network by computing the gradients $\nabla_{\theta} J(\theta)$ for each parameter $\theta$ and updating the parameters toward the direction of the gradient.

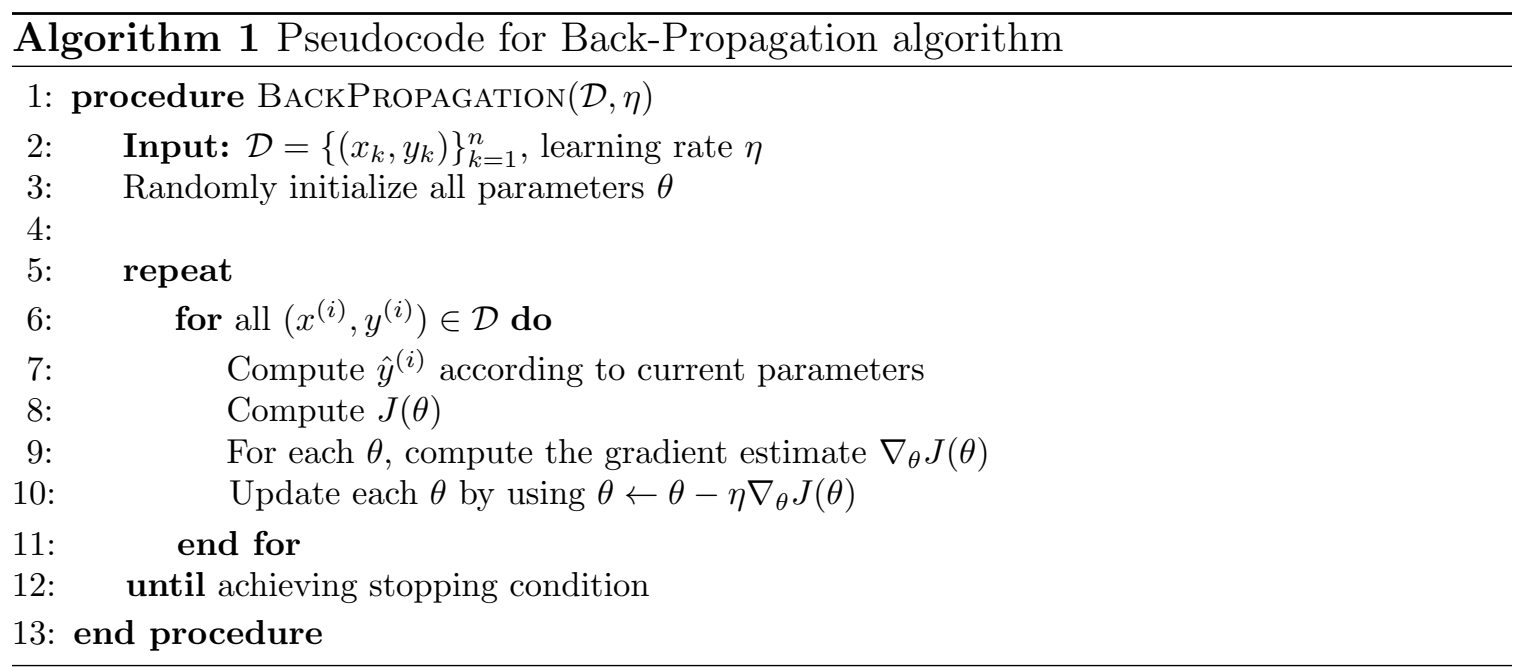

This optimization process is iterative and is continued until the model reaches a convergence point. 


\begin{tabular}{|c|c|c|c|c|c|c|c|c|c|c|c|c|c|}
\hline 0 & 1 & 1 & 1 & $\theta$ & 0 & 0 & & & & & & & \\
\hline 0 & 0 & 1 & 10 & $\left.1_{x}\right]$ & 0 & 0 & & & & 1 & 4 & 3 & $\begin{array}{lll}4 & 1\end{array}$ \\
\hline 0 & 0 & 0 & 1 & 1. & 1 & 0 & 1 & 0 & 1 & 1 & 2 & 4. & $3 \longdiv { 3 }$ \\
\hline 0 & 0 & 0 & 11 & 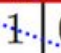 & 0 & 0 & 0 & 1 & 0 & 1 & 2 & $\begin{array}{ll}3 & 2\end{array}$ & \begin{tabular}{l|l}
4 & 1
\end{tabular} \\
\hline 0 & 0 & 1 & 1 & 0 & 0 & 0 & 1 & 0 & 1 & 1 & 3 & 3 & \begin{tabular}{l|l}
1 & 1
\end{tabular} \\
\hline 0 & 1 & 1 & 0 & 0 & 0 & 0 & & & & 3 & 3 & 1 & 100 \\
\hline 1 & 1 & 0 & 0 & 0 & & 0 & & & & & & & \\
\hline
\end{tabular}

Figure 2.3: An example of convolution operation in 2D space. Image taken from 3

\subsection{Convolution Neural Networks}

A convolution neural network $(\mathrm{CNN})$ is a special type of neural network used for processing data with spatial grid-like topology. Convolution as an operation is widely used in machine learning due to its fast computation for processing variable sized input data. More importantly, it leverages three important ideas [24] that can improve a machine learning system. First is the sparse connectivity, which is enforced by making the kernel smaller than the input. Second, convolution enables parameter sharing by using the same kernel weights operate over different sets of input representations. This helps to reduce the number of parameters in the whole model and makes the computation more efficient. Finally, due to the parameter sharing the operation tends to be equivariant to input representation translation.

Convolution is typically defined for a two-dimension space (Figure 2.3). This is generally the case because convolution is extremely popular for two-dimensional data such as images. In this thesis, we are particularly interested in applying convolution over one-dimensional data such as text sequences. To do so, will first have to define the one-dimensional case of the convolution operation. 
Definition 2.3.1. (One-dimensional Convolution Operation). Given an input matrix $x \in \mathbb{R}^{n \times d}$, the convolution operation over a single temporal dimension $n$ is defined as

$$
o_{i}=\sum_{j=1, c=1}^{k, d}\left[W_{j, c, 1} \cdot x_{i+j-\left\lceil\frac{k+1}{2}\right\rceil, c}, W_{j, c, 2} \cdot x_{i+j-\left\lceil\frac{k+1}{2}\right\rceil, c}, \ldots, W_{j, c, d} \cdot x_{i+j-\left\lceil\frac{k+1}{2}\right\rceil, c}\right]
$$

where $W \in \mathbb{R}^{k \times d \times d}$ is the learnable kernel with fixed pre-defined size $k, d$ is the dimension size of the representation and $o \in \mathbb{R}^{N \times d}$.

Here, we assume that the input representation matrix $x$ was appropriately zeropadded along the temporal dimension in both directions, in order to retain the original size of the dimension. This type of zero-padding is often called using the term "SAME" padding. Typically, when stride is equal to 1 the amount of padding for each side is given by $P=\left\lceil\frac{K-1}{2}\right\rceil$.

\subsubsection{Depthwise Convolutions}

The original form of convolution is well studied and highly optimized in both the software and hardware level. Over the years, researchers have put a lot of effort into finding less computationally intensive forms of convolutions to replace the standard convolution method. This is particularly pertinent when deploying neural models on edge devices where memory and computational power is limited. Depthwise convolutions have become very popular in edge intelligence applications as alternatives to convolution often yielding equivalent performance with less parameters. The difference between regular convolutions and depthwise convolutions is that the latter perform a convolution independently over every single channel (Figure 2.4). This helps reduce the number of parameters from $d^{2} k$ to $d k$ where $k$ denotes the kernel size. Next, we give the definition of the one-dimensional case of the depthwise convolution operation. 


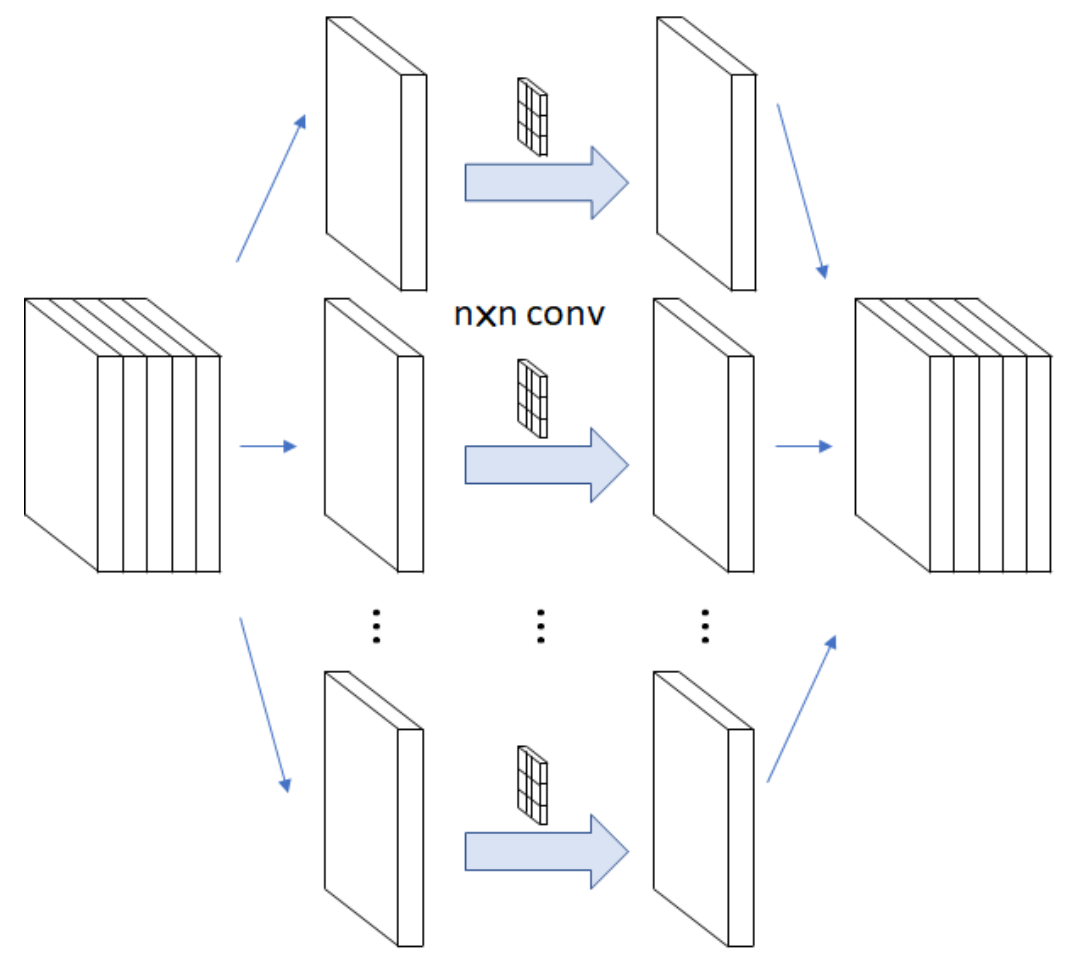

Figure 2.4: An example of depthwise convolution operation in 2D space. Image was taken from [4]. 
Definition 2.3.2. (One-dimensional Depthwise Convolution Operation). Given an input matrix $x \in \mathbb{R}^{n \times d}$, the depthwise convolution operation over a single temporal dimension $n$ is defined as

$$
o_{i}=\sum_{j=1}^{k} W_{j} \odot x_{i+j-\left\lceil\frac{k+1}{2}\right\rceil}
$$

where $W \in \mathbb{R}^{k \times d}$ is the learnable kernel with fixed pre-defined size $k$ and $o \in \mathbb{R}^{N \times d}$. Here $\odot$ denotes the element-wise multiplication between two vectors.

\subsubsection{Dilated Convolutions}

Vanilla convolutions struggle to integrate global context. The size of the receptive field of each layer (i.e. the block of pixels which can influence its activation) is $l *(k-1)+k$, where $l$ is the index of the layer. Practically this means that the effective receptive field of units can only grow linearly with layers. This is very limiting, especially for high-resolution input images. To overcome this issue, Yu et al. [5] proposed the dilated convolutions. Dilated convolutions are a way of integrating knowledge of a larger area (i.e. the global context of an image) while only linearly increasing the number of parameters. Figure 2.5 visualizes the dilation process of a dilated convolution.

Definition 2.3.3. (Dilated Convolutions). For a dilation size $l$, the kernel is subsampled every $l+1$ pixels, so a smaller kernel is "stretched" over a larger area. Given an input matrix $x \in \mathbb{R}^{n \times d}$, the dilated convolution operation over a single temporal dimension $n$ is defined as

$$
o_{i}=\sum_{j=1, c=1}^{k, d}\left[W_{j, c, 1} \cdot x_{i+l\left(j-\left\lceil\frac{k+1}{2}\right\rceil\right), c}, W_{j, c, 2} \cdot x_{i+l\left(j-\left\lceil\frac{k+1}{2}\right\rceil\right), c}, \ldots, W_{j, c, d} \cdot x_{i+l\left(j-\left\lceil\frac{k+1}{2}\right\rceil\right), c}\right],
$$




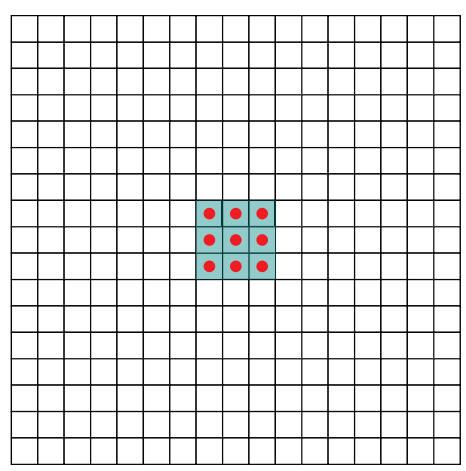

(a)

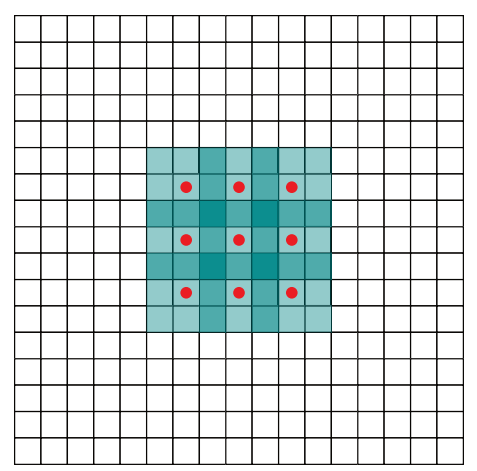

(b)

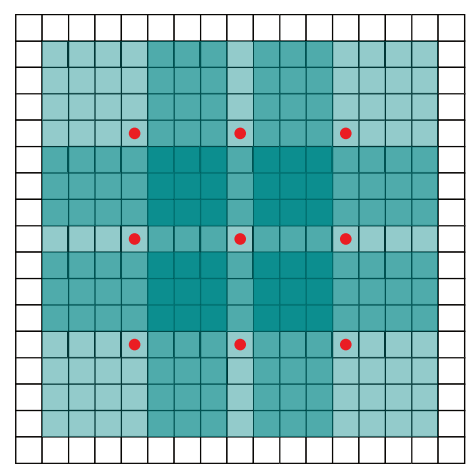

(c)

Figure 2.5: The dilated convolution operation. Subfigure (a) shows how normal convolution scans over an image. Subfigure (b) shows how dilated convolution with dilation size 2 has an effective receptive field of $7 \times 7$ while using a kernel of size $3 \times 3$. Subfigure (c) shows a dilated convolution with dilation size 4 . Figures taken from [5].

where $W \in \mathbb{R}^{k \times d \times d}$ is the learnable kernel with fixed pre-defined size $k, l$ is the dilation size and $o \in \mathbb{R}^{N \times d}$.

This way the receptive field of units grows exponentially across layers, thus it requires less layers (and parameters) to account for larger contexts.

\subsection{Recurrent Neural Networks}

A recurrent neural network $(\mathrm{RNN})$ is a special type of network that is used for encoding sequential data. A sequence is defined as a set on input representations that follow a temporal dependency between them. In other words, an RNN is an autoregressive model where the output representation depends linearly on its own previous output representations. A simple recurrent neural network is visualized in Figure 2.6 and below we give a formal definition of the recurrent unit. 


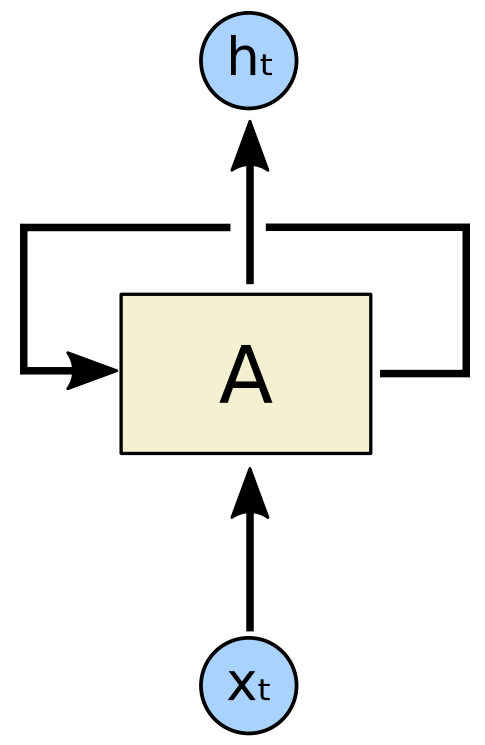

Figure 2.6: A recurrent neural network unit. The image is taken from [6].

Definition 2.4.1. (Recurrent Neural Network). A recurrent neural network is defined as $h^{(t)}=f\left(h^{(t-1)}, x^{t} ; \theta\right)$ where $t$ is the current time-step, $x^{t}$ is the input of the currenttime and $h^{t}$ and $h^{(t-1)}$ are the outputs of the current and the one step before time-steps respectively.

As indicated in [24], learning long-term dependencies in recurrent networks is mathematically challenging. Gradients that are propagated over many timesteps tend to either vanish or explode. In the first case, gradients become very small leading to no learning whereas in the second case the gradients become extremely large which drives the optimization process to overflow.

\subsubsection{Gated RNNs}

To mitigate the vanishing gradient problem, researchers have focused on the idea of creating paths through time that have derivatives that neither vanish nor explode. Specifically, they have introduced the notion of gate effectively creating a gated variant of RNNs. This gate unit is helping the neural network to forget the old recurrent 


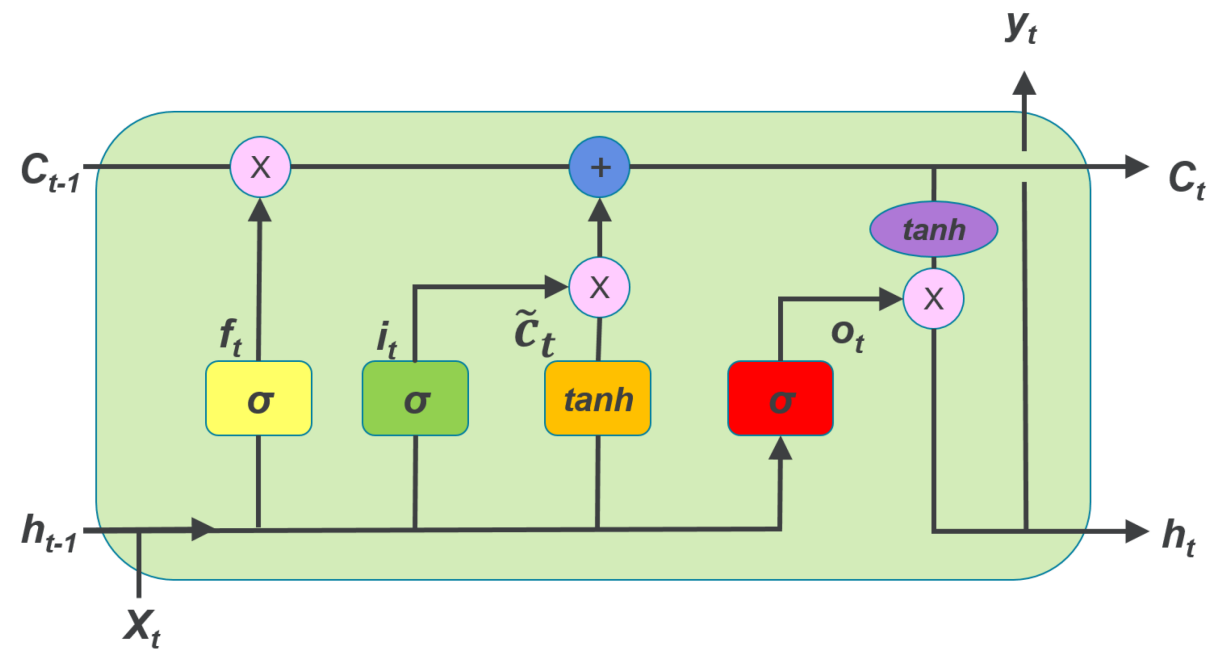

Figure 2.7: A long short-term memory unit. The figure is taken from 7].

state. This "forget" decision is learned implicitly by the network through the optimization process on the task and the data that it is trained on. The long short-term memory networks and the gated recurrent unit based networks are two of the most widely variations of gated RNNs used in the literature.

\section{Long Short-Term Memory Networks}

The Long Short-Term Memory (LSTM) network is a special type of gated RNN. The key of LSTMs is the introduction of the cell state. The cell state acts like an information highway that preserves a memory state between each time-step with few alterations on the representation. That being said, the hidden state through the addition of gates acts as short-term memory and the cell state as long-term memory between each temporal input step. Figure 2.7 visualizes the LSTM unit.

Definition 2.4.2. (Long Short-Term Memory). Given an input representation $x_{t}$ where $t$ is the current time-step, the output $h_{t}$ of the Long Short-Term Memory unit 
at the time-step $t$ is given by the following formulas

$$
\begin{aligned}
f_{t} & =\sigma\left(W_{f} \cdot\left[C_{t-1}, h_{t-1}, x_{t}\right]+b_{f}\right) \\
i_{t} & =\sigma\left(W_{i} \cdot\left[C_{t-1}, h_{t-1}, x_{t}\right]+b_{i}\right) \\
C_{t} & =f_{t} C_{t-1}+i_{t} \tanh \left(W_{C} \cdot\left[h_{t-1}, x_{t}\right]+b_{C}\right) \\
o_{t} & =\sigma\left(W_{o} \cdot\left[C_{t}, h_{t-1}, x_{t}\right]+b_{o}\right) \\
h_{t} & =o_{t} \tanh \left(C_{t}\right)
\end{aligned}
$$

where $W_{f}, W_{i}, W_{C}, W_{o}$ and their associated bias vectors are the learnable parameters of the unit. Here $\sigma$ denotes the sigmoid function and $f_{t}, i_{t}, C_{t}, o_{t}$ denote the forget gate, the input gate, the cell state, and the output gate respectively.

\section{Gated Recurrent Unit}

The need for all these gates that the LSTM network introduced has been questioned by many researchers in the area. The most successful gated RNN alternative to LSTMs has been networks based on the Gated Recurrent Unit (GRU). The main difference with the LSTM is that in GRU the single gating unit controls both the forgetting factor and the decision to update the state unit at the same time. For a graphical representaion of the GRU, refer to Figure 2.8.

Definition 2.4.3. (Gated Recurrent Unit). Given an input representation $x_{t}$ where $t$ is the current time-step, the output $h_{t}$ of the Gated Recurrent Unit at the time-step 


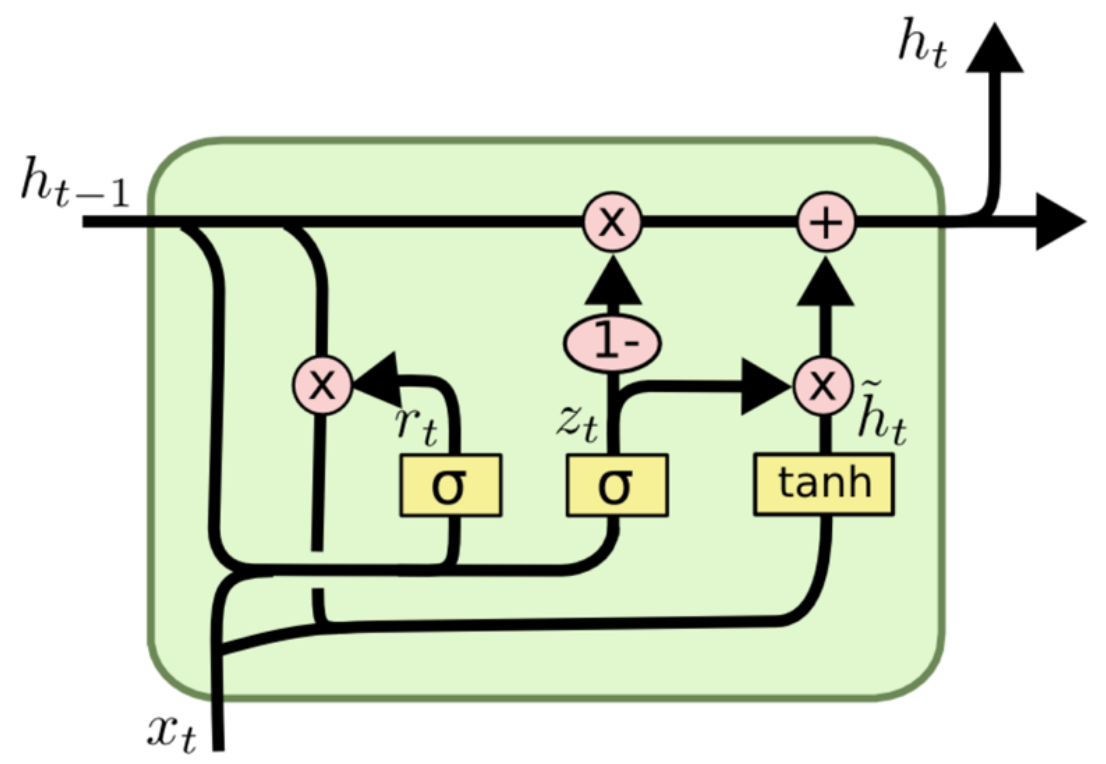

Figure 2.8: A gated recurrent neural network unit. The image is taken from 6 .

$t$ is given by the following formulas

$$
\begin{aligned}
z_{t} & =\sigma\left(W_{z} \cdot\left[h_{t-1}, x_{t}\right]\right) \\
r_{t} & =\sigma\left(W_{r} \cdot\left[h_{t-1}, x_{t}\right]\right) \\
\hat{h}_{t} & =\tanh \left(W \cdot\left[r_{t} h_{t-1}, x_{t}\right)\right. \\
h_{t} & =\left(1-z_{t}\right) h_{t-1}+z_{t} \hat{h}_{t}
\end{aligned}
$$

where $W_{z}, W_{r}, W$ and their associated bias vectors are the learnable parameters of the unit. Here $\sigma$ denotes the sigmoid function and $z_{t}, r_{t}$ denote the update gate and the reset gate respectively.

\subsubsection{Bidirectional RNN}

A typical recurrent network has a causal structure, which means that a hidden state at the time-step $t$, considers only information from the current $x_{t}$ and the past 
$\left\{x_{1}, \ldots, x_{t-1}\right\}$ input representations. In some applications such as text comprehension, we need to encode the current time-step based on the whole input sequence.

Definition 2.4.4. (Bidirectional RNN). A bidirectional recurrent neural network is a network where the recurrent layer consists of two recurrent units, the forward and the backward unit. The forward unit is responsible of scanning the input sequence from the beginning to the end of the sequence. On the other hand, the backward unit scans the sequence from the end to the start of the sequence. The two output representations for each directions are stacked together to form the final layer output.

\subsection{Attention}

In this section, we introduce the notion of attention in deep learning. Attention is a key element of modern approaches to sequence learning and has shaped the current state-of-the-art directions.

Definition 2.5.1. (Attention). Attention is the operation that selects the largest element from some set $X$, where the notion of what is considered to be the "largest" is represented by some set $S$ of scores. Since every function in a neural network has to be differentiable, we cannot use the $\arg \max (\cdot)$ function to select the element with the highest score $S_{i}$. Instead, we generate a categorical distribution over the elements of $X$ using the $\operatorname{softmax}(\cdot)$ function. The following formula defines the attention operation.

$$
\hat{X}=X \cdot \operatorname{softmax}(S)
$$

Here the set $S=\left\{f\left(y_{i}\right)\right\}$ where $f: \mathbb{R} \rightarrow \mathbb{R}$ is a score function that assigns a score to each $y_{i} \in Y$. Each $y_{i}$ is some evidence according to which a particular $x_{i}$ is to be selected. Since $f$ is a function, we can learn it (e.g. represent it as a neural network 
with some parameter $\theta$ ).

$$
S=f\left(Y^{T} ; \theta\right)
$$

In the degenerate case when $X=Y$ the operation is called self-attention.

\subsection{Word Embedding and Subword Tokenization}

In this thesis, we are particularly interested in applying sequence modeling techniques to text data. A text sequence is nothing more that a sentence composed by words from a specific language. In natural language processing, a word is represented as an one-hot vector over the vocabulary-sized space. This vector contains the value one on the corresponding word and zero everywhere else. However, representing a word in this manner is not applicable in deep learning approaches. This is because the one-hot vector only contains the index of the word and no actual information that describes what the word means. In deep learning models, it is common to represent each word with its own latent vector of length $d$. These vectors can be jointly optimized with the rest of the model and can thus, implicitly capture the latent information of the meaning of the words relevant to the learning task and the available training data. Stacking all these word vectors together creates the word embedding matrix $E \in \mathbb{R}^{\mathcal{V} \times d}$ where $\mathcal{V}$ is the size of the vocabulary.

Segmenting a sentence based on words has been the standard text process for many years. That being said, word-based tokenization has two main issues. First, word embedding models are limited by vocabulary size and the frequency of word occurrences. In other words, rarely used words would never be explicitly captured and when they did occur in a text, they would be assigned a special word type, which we call unknown $(<\mathrm{UNK}>)$. The second issue that can arise is that when we are dealing with word embedding matrices for multiple languages, the size of the 
vocabulary that we would need to support all languages would increase dramatically. Storing such a high-dimensional matrix would be impractical.

The last few years, researchers have put a lot of effort into trying to find alternative tokenization methods and character-based embedding matrices were the natural alternative approach to consider. The reduction of the vocabulary size is significant since we are breaking the sentence into the primitive characters of the corresponding language. The issue with this approach is that by breaking the sentence into so many tokens (characters), we drastically increase the long-term dependency that our sequence model will have to optimize for. As of the time of writing, learning very long-term dependencies is still an active research area. In addition, optimizing a character representation to learn all its possible meanings and combinations puts a lot of pressure on the rest of the model to capture this information. This leads to a model with huge network capacity (i.e. with many millions of parameters) which is obviously not very practical.

Today, subword tokenization schemes inspired by Byte Pair Encoding (BPE) 25 have become the norm in most advanced NLP models. Subword tokenization brings the perfect balance between character-based and word-based representations. BPE works by finding the most frequent character $n$-grams between a vocabulary based on words. The user defines the desired subword-based vocabulary size $k$ and the algorithm returns the top- $k$ most frequent subwords. To maximize the coverage of all possible input words, the primitive language characters in the vocabulary are included so in case subwords that perfectly match the word cannot be found, the word can then be split by its characters instead. 


\subsection{Text Generation}

Text generation is one of the core tasks in natural language processing. Afterall, the main goal of Language Modeling (LM) is to derermine the probability $P$ or likelihood of a sequence of tokens $W$ (Figure 2.9).

$$
P(W)=P\left(w_{1}, w_{2}, \ldots, w_{N}\right)
$$

where $w_{1}$ is the first token of the sequence and $N$ is the total number of tokens in the sequence.

The above joint probability can be decomposed into a product of conditional probabilities using the chain rule of probability.

$$
\begin{aligned}
P\left(w_{1}, w_{2}, \ldots, w_{N}\right) & =P\left(w_{1}\right) P\left(w_{2} \mid w_{1}\right) \ldots P\left(w_{N} \mid W_{1}, \ldots, w_{N-1}\right) \\
& =P\left(w_{1}\right) \prod_{i=2}^{N} P\left(w_{i} \mid w_{1}, \ldots, w_{i-1}\right)
\end{aligned}
$$

where $i$ is equal to the time-step. The probability $P\left(w_{1}\right)$ is the probability of seeing the token $w_{1}$ at the beginning of the generated sequence if there is not previous context given. Thus, a language model can be used to measure the probability of a sequence of text, in the sense that a sequence that is more likely to occur in a certain language will have a higher probability than an unlikely sequence.

\subsubsection{Conditioned Text Generation}

The goal is often to generate text, based on some prior condition, as for example, in the case of Neural Machine Translation (NMT), where in order to start generating a translated sentence the sentence needs to first be processed in its original language. The most common framework for achieving this is the Sequence-to-Sequence scheme 


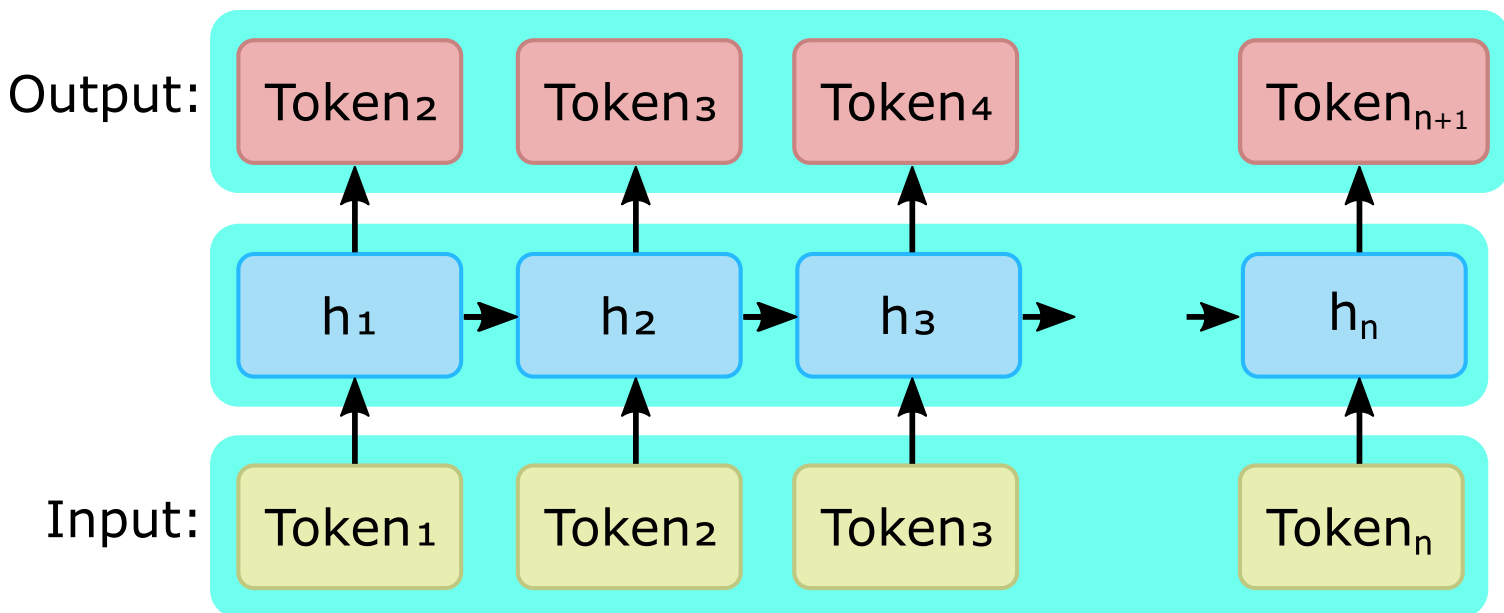

Figure 2.9: The general framework of a language model.

(Seq2Seq) [8].

Given a source sequence representation vector $s$, we condition the output token probabilities as

$$
P(W \mid s)=P\left(w_{1}, w_{2}, \ldots, w_{N} \mid s\right)
$$

This means that the Equation 2.17 becomes

$$
\begin{aligned}
P\left(w_{1}, w_{2}, \ldots, w_{N} \mid s\right) & =P\left(w_{1} \mid s\right) P\left(w_{2} \mid w_{1}, s\right) \ldots P\left(w_{N} \mid W_{1}, \ldots, w_{N-1}, s\right) \\
& =P\left(w_{1} \mid s\right) \prod_{i=2}^{N} P\left(w_{i} \mid w_{1}, \ldots, w_{i-1}, s\right)
\end{aligned}
$$

In the deep learning literature, it is common to implement the Seq2Seq scheme using the Encoder-Decoder model architecture proposed by Cho et al. [26]. The general architecture of an encoder-decoder model is agnostic of the sequence learning architecture that is used. Specifically, the encoder and decoder modules are modelled using any of the available sequence encoding methods such as LSTM, GRU, convolution and attention based methods. 


\subsection{Beam Search}

When a text sequence is generated, the model is essentially predicting the output probability distribution over the vocabulary $\mathcal{V}$ space for each time-step. The obvious way of selecting which token to choose from this output distribution is to select the token with the highest probability, a procedure that is referred in the literature as greedy search. The issue with this approach is that we can potentially end up having a sequence with low overall probability compared to some other candidate sequences. For this reason, it is a common practice to use alternative search methods to sample from the output distribution that maximize the sequence probability.

The most popular approach among all the alternative search methods is beam search. Beam search allows for non-greedy local decisions that can potentially lead to a sequence with a higher overall probability. The algorithm requires the user to set a beam size or width $B$. This value is responsible of controlling the maximum number of sequences that the algorithm will expand at each time-step. When the beam size is set to the vocabulary size, then the algorithm is exhaustively searching to find through all possible sequence the one with the highest overall probability. This of course is impractical because the time complexity is $O\left(N \cdot \mathcal{V}^{2}\right)$, thus the value is usually set to a number between 4 and 10 which has been shown to give relatively good results. In contrast to greedy search where the time complexity of the algorithm is $O(N \cdot \mathcal{V})$ where $N$ is the length of the generated sequence, beam search has time complexity $O(B \cdot N \cdot \mathcal{V})$ which makes it slower as the beam size increases. Algorithm 2 shows the pseudocode of beam search. 


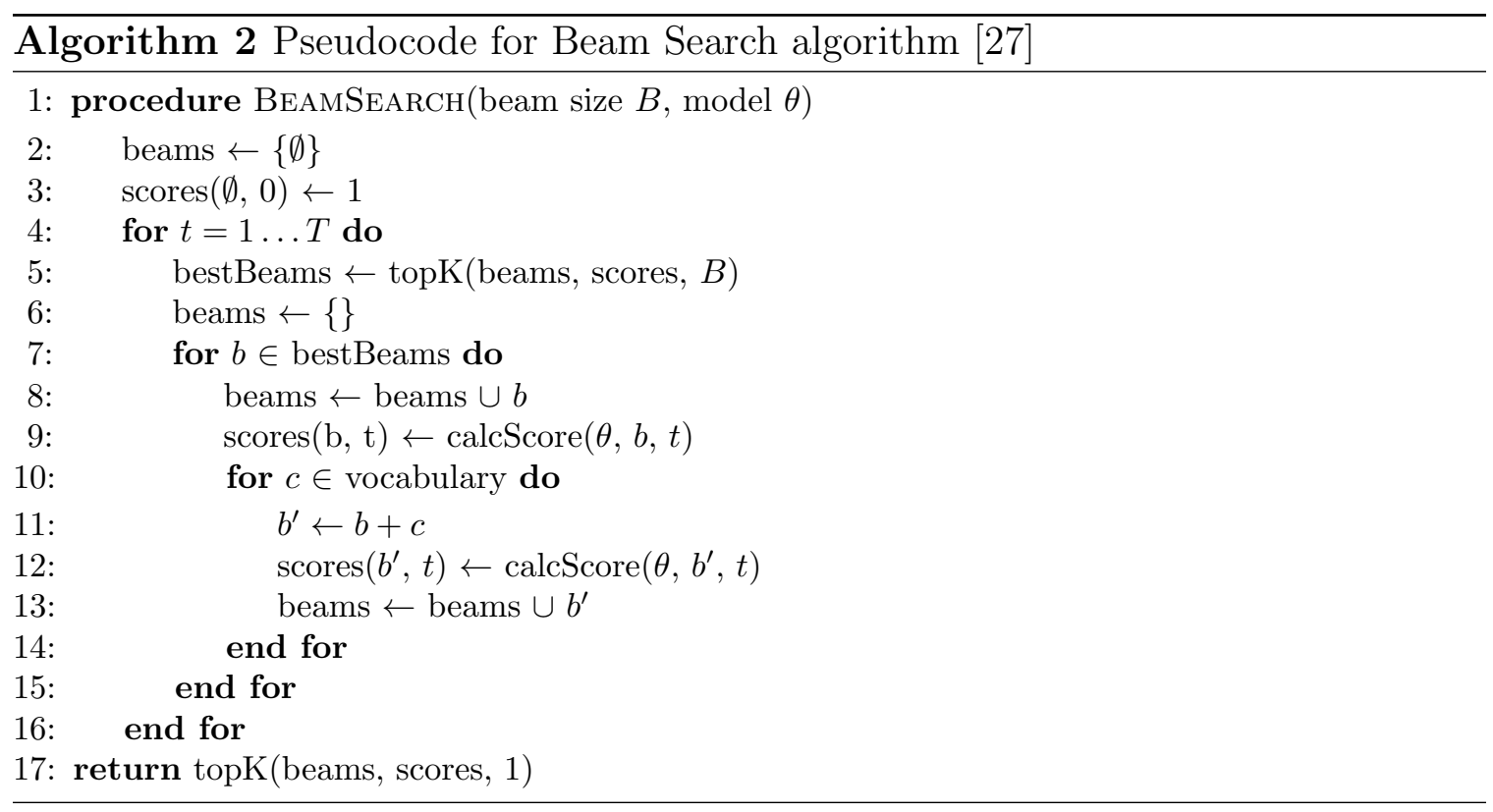

\subsection{Evaluation Metrics}

In this section, we introduce the different evaluation metrics that we will use through out the thesis. Our proposed sequence learning method will be evaluated on four natural language processing tasks namely, neural machine translation (NMT), language modeling (LM), abstractive text summarization and sentence classification.

\subsubsection{BLEU- $n$ Score}

Machine translation is a challenging task and as such, it has been studied for years. The translation model, given a sentence in the source language, should be able to generate a corresponding good translation in the target language. Training a neural machine translation model using supervised learning, presuppose that we have data pairs of sentences in the source and target language. Evaluating a generated translation is difficult due to the stochastic nature that human languages follow which entails that more than one translation can be considered correct. 
Researchers in the field, have experimented with various different ways to automatically evaluate the generated translation without the need of human experts. Nonetheless, this is still an open problem. The most widely used metric employed for this task as of today is BLEU- $n$ score. BLEU stands for Bilingual Evaluation Understudy and is a geometric average precision over 1 - to $n$-grams multiplied by a brevity penalty for short sentences. The score range is between zero (no overlapping between generated and reference translations) and one (generated and reference translations completely match).

Definition 2.9.1. ( $n$-gram). An $n$-gram is a contiguous sequence of $n$ items from a given sample of text.

Most neural machine translation methods in the literature evaluate their approaches using BLEU-4. This means that the method is evaluated based on its precision when generating 1-grams, 2-grams, 3-grams and 4-grams against the reference translation sentence.

\subsubsection{ROUGE Score}

In abstractive text summarization, the objective is to encode a corpus text in a latent representation and conditionally on this representation, generate a significantly shorter sequence that summarizes over the overall meaning of the corpus text. Abstractive summarization models in the literature are being evaluated using the ROUGE (Recall-Oriented Understudy for Gisting Evaluation) score. This score is measuring the $n$-gram recall between the candidate summary sequence and the reference abstract sequence.

It is common to report three different metrics using the F-score of ROUGE. The first one is ROUGE-1 which refers to the overlap of unigram (each token) between 
the generated and reference summaries. The second is ROUGE-2, which refers to the overlap of bigrams between the generated and reference summaries. Finally, ROUGE-L is based on the longest common subsequence problem and takes into account sentence level structure similarity naturally and identifies longest co-occurring in sequence $n$-grams automatically.

\subsubsection{Perplexity}

The concept of perplexity has its origin in information theory. It measures the fitness of a probability distribution when predicting a given sample. Generally speaking, the lower the perplexity, the better the probability distribution is at predicting the sample. In natural language processing, perplexity is a way of evaluating language models. A language model is a probability distribution over entire sentences. In NLP, perplexity is defined as the inverse probability of the test set, normalized by the number of tokens.

Specifically, given the probabilities of each generated token, we compute the perplexity with

$$
\begin{aligned}
P P(W) & =P\left(w_{1} w_{2} \ldots w_{N}\right)^{-\frac{1}{N}} \\
& =\sqrt[N]{\frac{1}{P\left(w_{1} w_{2} \ldots w_{N}\right)}}
\end{aligned}
$$

which equivalently can be expressed as

$$
P P(W)=2^{-l}
$$

where

$$
l=\frac{1}{N} \log P\left(w_{1} w_{2} \ldots w_{N}\right)
$$




\subsubsection{Classification Accuracy}

In sequence classification, the main goal is to train a sequence encoding model that is capable of comprehending a text input sequence and producing an output distribution over the number of prediction classes. Classification accuracy is the standard evaluation metric for classification tasks and it measures the number of correct predictions among all predicted test samples. It is defined as the ratio of the number of correct predictions over the total number of input samples.

$$
\text { Accuracy }=\frac{\text { Number of Correct predictions }}{\text { Total number of predictions made }}
$$




\section{Chapter 3}

\section{Related Work}

\subsection{Introduction}

The sequence modeling task using neural networks is about using specialized neural architectures that can exploit the different combinations of the time-steps of a sequence in order to form higher level representations. This task is considered one of the fundamental tasks in machine learning. In this section, we introduce a brief review of the main neural network approaches that have been proposed in the empirical literature to model sequences. Since our proposed method is using a novel adaptive convolution operation, we also provide insight on how recently some works have started working on dynamically enlarging the receptive field of convolution networks.

\subsection{Sequence Modeling}

As it was previously mentioned, sequence modeling is one of the core tasks in machine learning. It involves a neural model capable of both encoding and comprehending a sequence as well as generating a sequence. To date, there are three families of sequence modeling approaches. The first is recurrent-based methods, the second 
is convolutional-based approaches and the third is models based on self-attention. In this section we will introduce the main contributions in each sequence modeling category.

\subsubsection{Recurrent-based Methods}

Recurrent-based methods dominated for many years the world of sequence modeling theory. Recurrent neural network based encoder/decoder approaches were the first to naturally learn to encode and generate sequences. In general, an encoder based on RNNs will take as an input a sequence $x=\left\{x_{1}, \ldots, x_{s}\right\}$ of $s$ elements. This RNN model will return the state representations $\bar{h}=\left\{\bar{h}_{1}, \ldots, \bar{h}_{s}\right\}$ for each $x_{i}$ element. The decoder RNN-based model will take $h$ and generate the output sequence $\hat{y}=\left\{\hat{y}_{1}, \ldots, \hat{y}_{t}\right\}$, one element at a time. The decoder for each timestep computes a conditional input $c_{i}$. This conditional input is obtained from the encoder output representations $\bar{h}$. To generate a new output $\hat{y}_{i+1}$, the decoder takes as input the previous hidden state $h_{i}$, the conditional encoder input $c_{i+1}$ and a representation of the previously generated timestep $f\left(\hat{y}_{i}\right)$. This constitutes a generic formulation of the approach that almost all other recurrent-based methods follow. The main contributions of works using RNN are mainly related to the strategy of computing the conditional input $c_{i}$ and the type of the RNN architecture they employ.

\section{Seq2Seq Learning with LSTM Networks}

The work of Sutskever et al. [8] is the first to use neural networks for sequence learning. Specifically, the method (Figure 3.1) consists of multiple stacked LSTM networks acting as the encoder model. This encoder model maps the input sequence to a vector of a fixed dimensionality. Next, another set of multiple stacked LSTM networs denoted as the decoder model, is responsible to decode to the target text sequence. This is done by providing the fixed source context vector as the initial 


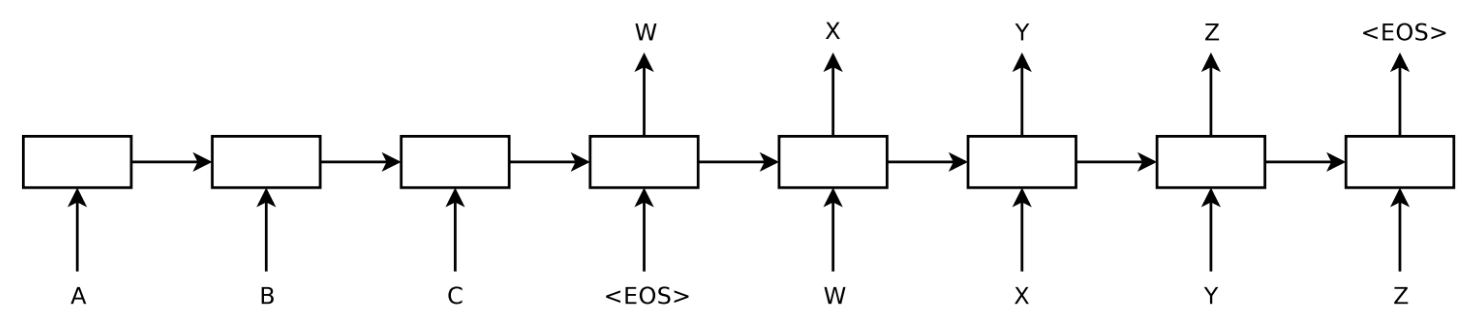

Figure 3.1: The first seq2seq LSTM-based approach. 8

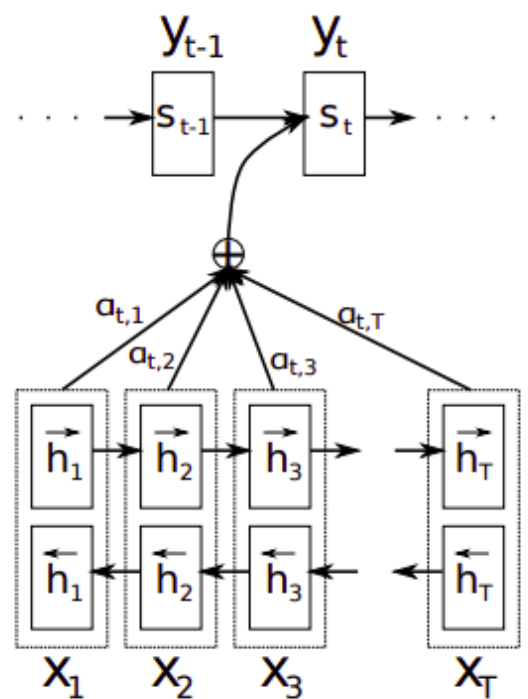

(a) Bahdanau et al. [17 approach using attention with LSTMs.

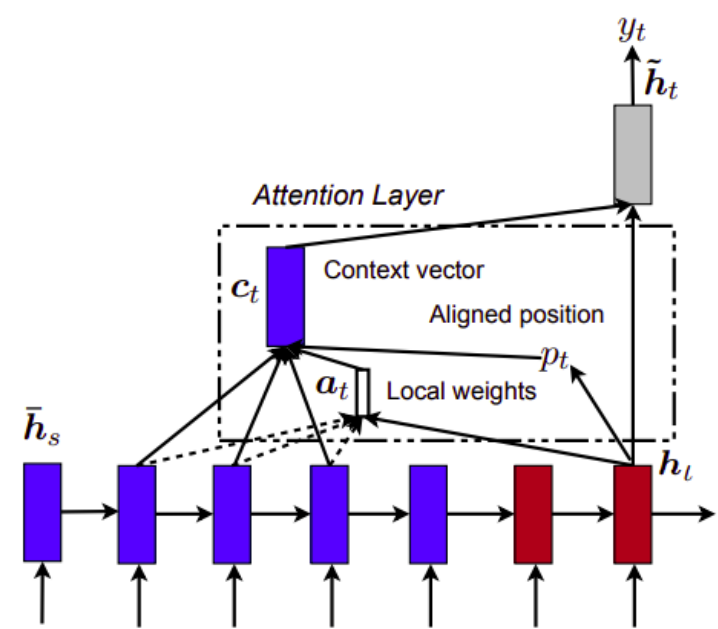

(b) Local attention model proposed by Luong et al. 28].

Figure 3.2: Attention with LSTM-based approaches.

hidden state to the decoder LSTMs. This work ignited the spark of the revolution of employing neural networks in the area of NLP and helped shape the at the time, state-of-the-art results in neural machine translation.

\section{Attention with LSTM Networks}

Bahdanau et al. [17], argued that the use of a fixed-length vector of the last time-step is a bottleneck in improving the performance when provided as the initial hidden state to the decoder network. For this reason, they introduced a soft-selection (attention) 
over all of the encoded time-steps. The attention is calculated based on the current hidden state from the decoder and across all the encoder's representations. These scores are then multiplied with each encoded representation of the input sequence and aggregated together forming the final hidden state that the decoder will use for the next time-step. In addition, the encoder network consists of bidirectional LSTM networks which help to better capture the overall meaning of the input sentence. Figure $3.2 \mathrm{a}$ shows a graphical illustration of the architecture.

Next, Luong et al. [28], took the idea of attention one step further. Their work introduced the notion of the local attention model (Figure 3.2b). This attention module first predicts a single aligned position $p_{t}$ for the current target word. Following this, a window is centered around the source position $p_{t}$ and the tokens in this window are used to compute a context vector $c_{t}$ using a weighted average of the source hidden states in the window. Finally, the attention scores $a_{t}$ are inferred from the current target state $h_{t}$ and those source states $\bar{h}_{s}$ in the window.

\section{GRU-based Architectures}

Cho et al. [26], developed an alternative to LSTMs recurrent architecture, which is much simpler to both compute as well as implement. This recurrent unit is called GRU and it has already been introduced in a previous Section 2.4.1. Since Cho and colleagues published their work, numerous other papers have utilized various combinations of GRUs and attention [29, 30] as well as variants of the architecture 31,32 .

\section{Training Deeper Recurrent Models}

Typically, models with multiple layers are difficult to train. This is due to the vanishing/exploding gradients problem as well due to the degradation problem where the 


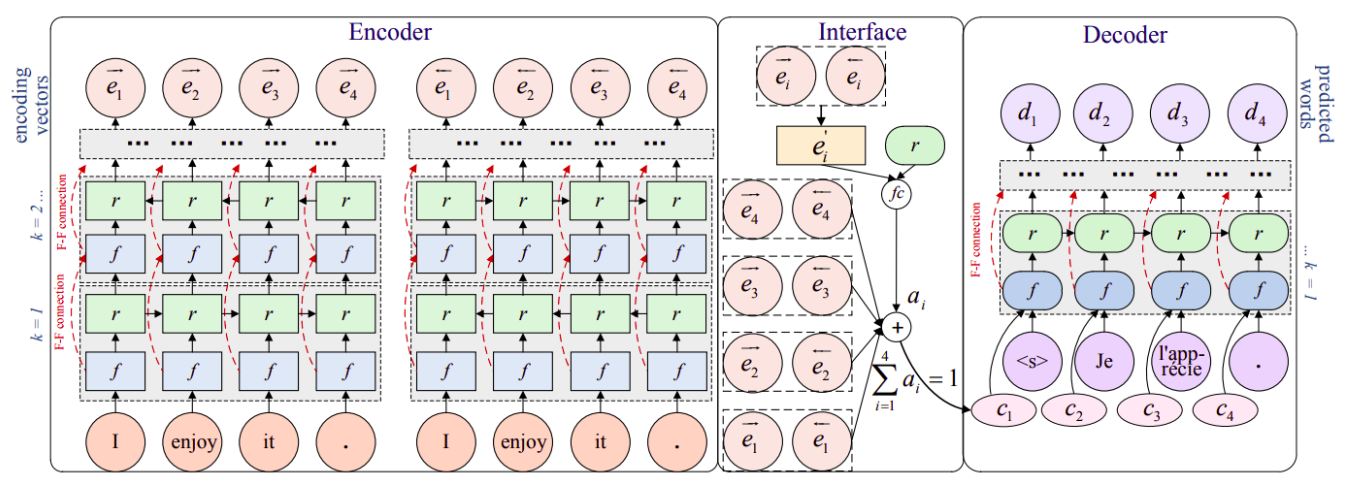

Figure 3.3: A very deep LSTM-based neural machine translation architecture proposed by He et al. [9].

accuracy gets saturated and then degrades rapidly. This phenomenon is widely studied by He et al. [9] where he proposed the skip connection "trick" to mitigate these issues. Based on the idea of residual connections, Zhou et al. [33 proposed a very deep LSTM-based neural machine translation architecture (Figure 3.3) which significantly improved the translation performance. The research team from Google in 2016 conducted a large scale experimentation [18 with an LSTM-based model composed by 16 layers in total connected with skip connections. The team found that it is possible to train an extremely deep recurrent-based model that yields state-of-the-art results.

\subsubsection{Convolution-based Methods}

Convolution-based approaches are less common for sequence modeling. Convolutions usually represent a timestep using a fixed size context. The effective context size of the overall model can be made larger by introducing several layers that make the model deeper. This can allow the designer of the sequence model to control the maximum length of the dependencies that are going to be modeled. In addition, due to the fact that convolution methods are non-autoregressive and the computation of the current timestep does not depend on the previous timesteps, the convolution-based 


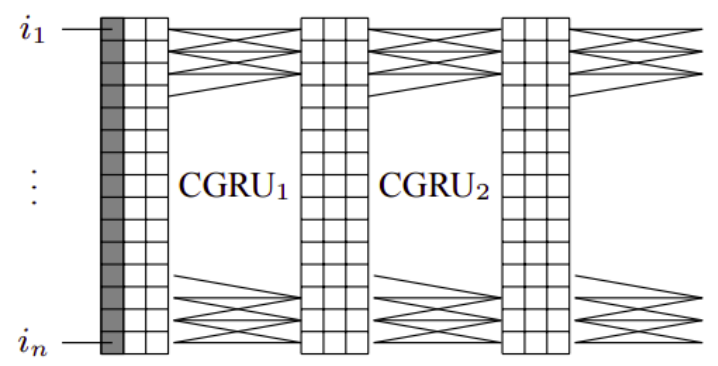

$s_{0}$

$s_{1}$

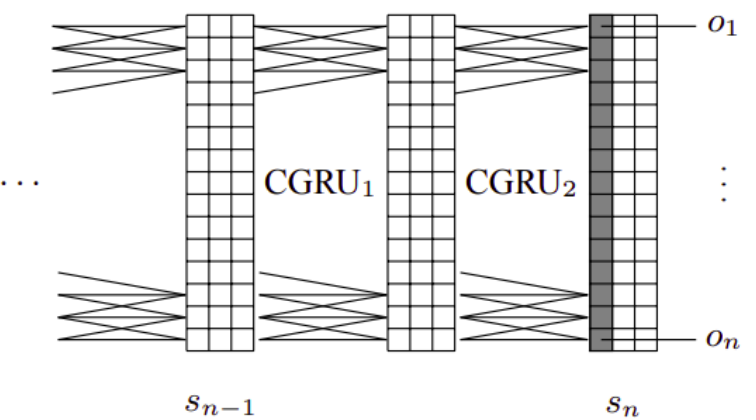

Figure 3.4: The first convolution-based sequence modeling approach proposed by Kaiser et al. [10].

approaches allow parallelization over every element in a sequence.

\section{Convolutional Gated Recurrent Networks}

In 2015, Kaiser et al. [10] proposed for the use convolutions for modeling and generating sequences for the first time. More specifically, they proposed the Convolutional Gated Recurrent Networks (CGRNs), a special type of a GRU unit where each linear projection layer is replaced by a convolution operation. This network was the catalyst factor for the empirical research to strive for faster, more parallelizable alternatives to recurrent-based approaches. Kaiser et al. [10] showed that with their approach multiple parallel operations are able to be performed in each step, and the method is graphically represented in Figure 3.4 .

\section{ByteNet Architecture}

The ByteNet was proposed by Kalchbrenner et al. [21] and it is an architecture for neural machine translation which translates in linear time and can handle dependencies over large distances. The sequence encoding unit is formed of one-dimensional convolutional layers that use dilation. The network is using a method called Dynamic Unfolding. Specifically, the representation generated by the source network 
has the same length as the source sequence. At each step, the target network takes the corresponding column from the source representation and generates an output. This continues until an end-of-sequence (EOS) symbol is produced by the target network. The source representation is automatically zero-padded as the steps go beyond its length and the output is conditioned on the source and target representations accumulated thus far. The ByteNet architecture is computationally expensive and requires a lot of parameters.

\section{Convolutional Sequence to Sequence Learning}

Perhaps, the most famous work involving convolutions with sequence learning is proposed by Gehring et al. [11]. This is the first work that utilizes convolutions as a standalone replacement to a recurrent network. Previous work replaced linear operations with convolutions while still maintaining the recurrent behaviour. The process of Gehring is shown in Figure 3.5 .

The first novelty that this method introduced is the addition of the position embedding. Since the method is not recurrent, the model has no information about the ordering of the input tokens. Thus, adding a positional representation to the input embedding representation would allow the model to learn and associate the ordering of each input token. The second innovation involves the use of the convolution operation over a fixed window of tokens. This includes a smart zero-padding process on the decoder input representation that allows to skip over future token representations and take only into account the current and the past tokens. The third novelty is the use of multi-step attention, where each decoder layer performs a dot-product attention with the output representations from the encoder network. 


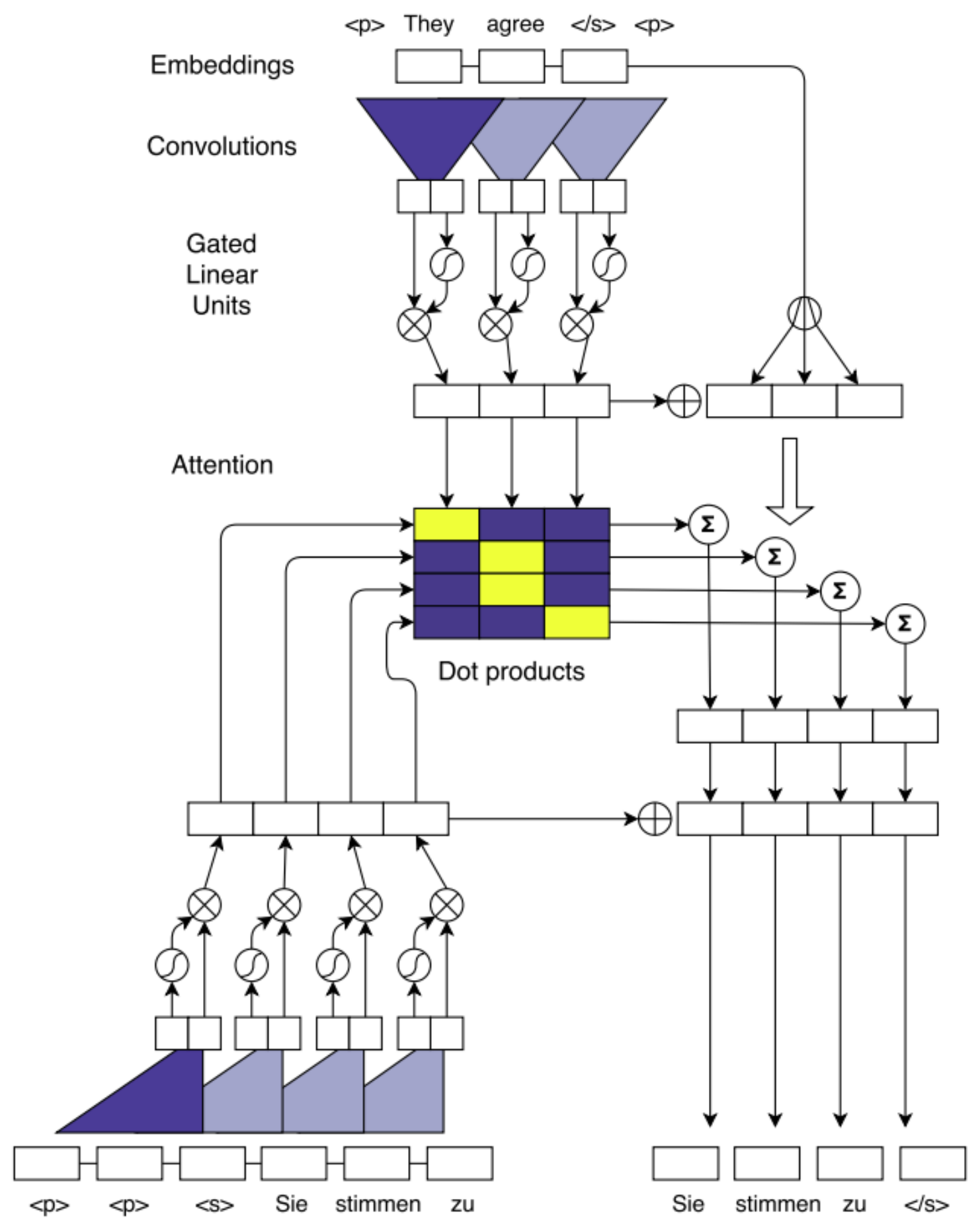

Figure 3.5: The first convolution-based sequence modeling approach that is based only on convolutions in a non-autoregressive way. The image was taken from 11. 


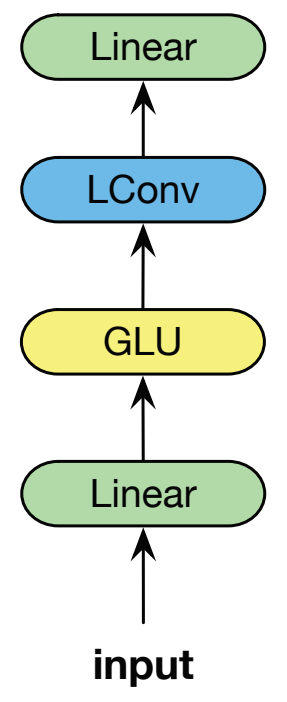

(a) Lightweight Convolution.

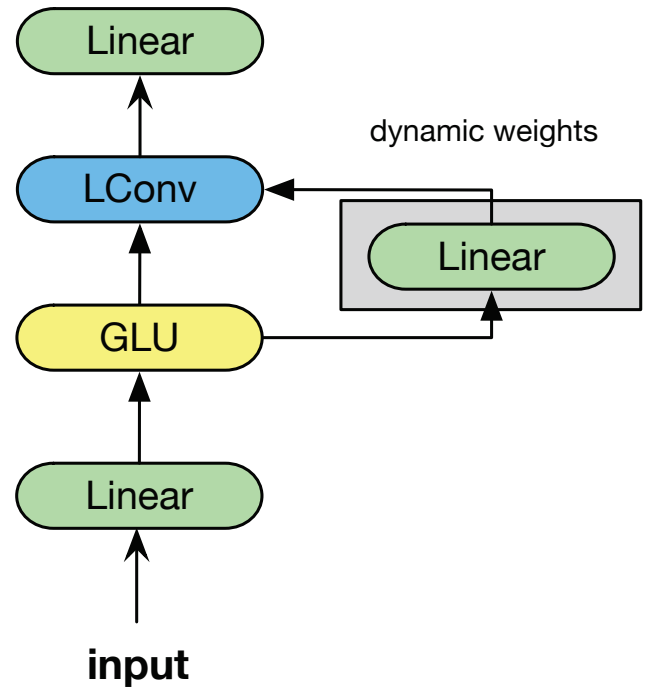

(b) Dynamic Convolution.

Figure 3.6: Lightweight and Dynamic convolution units proposed by Wu et al. [12.

\section{Lightweight and Dynamic Convolutions}

In 2019, Wu et al. [12] extended the convolution sequence to sequence operation by introducing the use of depthwise convolutions. More specifically, they proposed the Lighweight convolution unit (Figure 3.6a) as a replacement for any sequence modeling operation. A lightweight convolution unit is composed by a linear projection followed by a Gate Linear Unit (GLU) [34]. The output is then passed to a depthwise convolution with a learnable kernel. The kernel of the convolution operation is passed through a softmax normalization before it is applied. Finally, another linear projection is used to bring the representation to the same space as the input representation and perform a skip-connection between the two representations.

The lightweight convolution learns a fixed sized kernel which is used for all input sequences. $\mathrm{Wu}$ et al. tried to extend this idea by utilizing a dynamically generated kernel for each input sequence. Specifically, a linear projection would take the input representations and generate a separate softmax normalized kernel for each sequence 


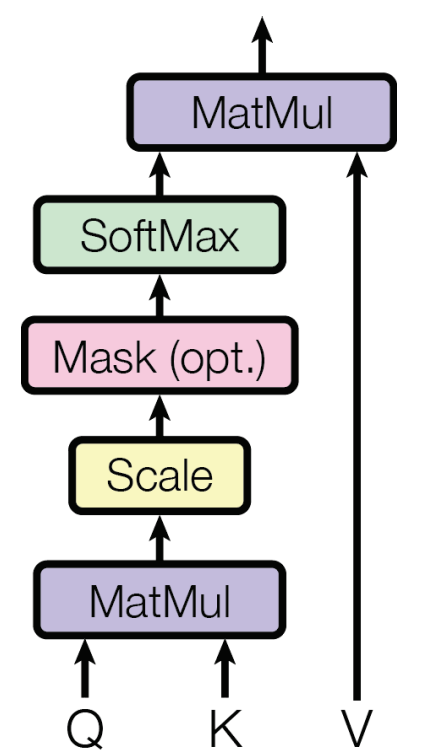

(a) The Scaled Dot-Product Self-Attention.

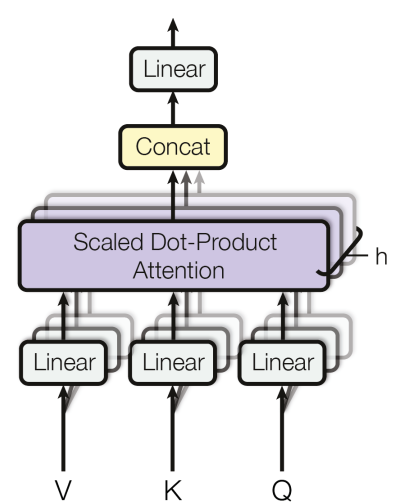

(b) Multi-headed Self-Attention.

Figure 3.7: The Transformer's multi-head self-attention unit. The image was taken from 13 .

segmentation. They called this convolution operation Dynamic convolutions (Figure 3.6b. This work showed that you do not have to encode a time-step representation using all available tokens in order to achieve state-of-the-art results.

\subsubsection{Attention-based Methods}

The attention mechanism helped the recurrent-based approaches to further raise the bar and achieve state-of-the-art results. For the first time in 2017, a new approach was proposed, the Transformer network, that used self-attention to directly model a sequence in a non-autoregressive way. Since then, self-attention based methods became the standard sequence modeling direction that any modern state-of-the-art solution employs, especially when it comes to natural language processing applications. 


\section{Self-attention and the Transformer Network}

Today, almost every state-of-the-art sequence modeling approach employs a variant of the Transformer network. Transformers are based on the concept of self-attention and were originally proposed by Vaswani et al. [13]. Specifically, each time-step of the input sequence is transformed using a linear projection layer into three matrices called query, key and value. Each query vector is then multiplied with each key vector. Next, the product is passed through a softmax function, which creates the attention scores. These scores show how to combine (based on the attention distribution) all the keys/values for each vector (time-step) of the query matrix. Self-attention is the case when query and key are using the same input representations (See Figure 3.7a). Alternatively, when query is different from keys (i.e. encoder-decoder attention) then it simply called attention.

An additional innovation of the transformer network was the introduction of multihead attention (Figure 3.7b). Instead of performing a single attention for the whole $d$ dimensional space of the input representations, the space $d$ is organized into $H$ groups (heads) and attention is performed for each subspace separately. Multi-head attention allows the model to jointly attend to information from different representation subspaces at different positions. Figure 3.8 shows the overall architecture of the

Transformer network. To date, transformers are widely used in the NLP area and are considered to be the standard approach for modeling sequences.

\section{Transformer-XL}

Transformers are very popular mainly due to their ability to capture long-term dependencies better. However, the vanilla implementation of Transformers uses a fixed-length context. This means that a long text sequence is truncated into 


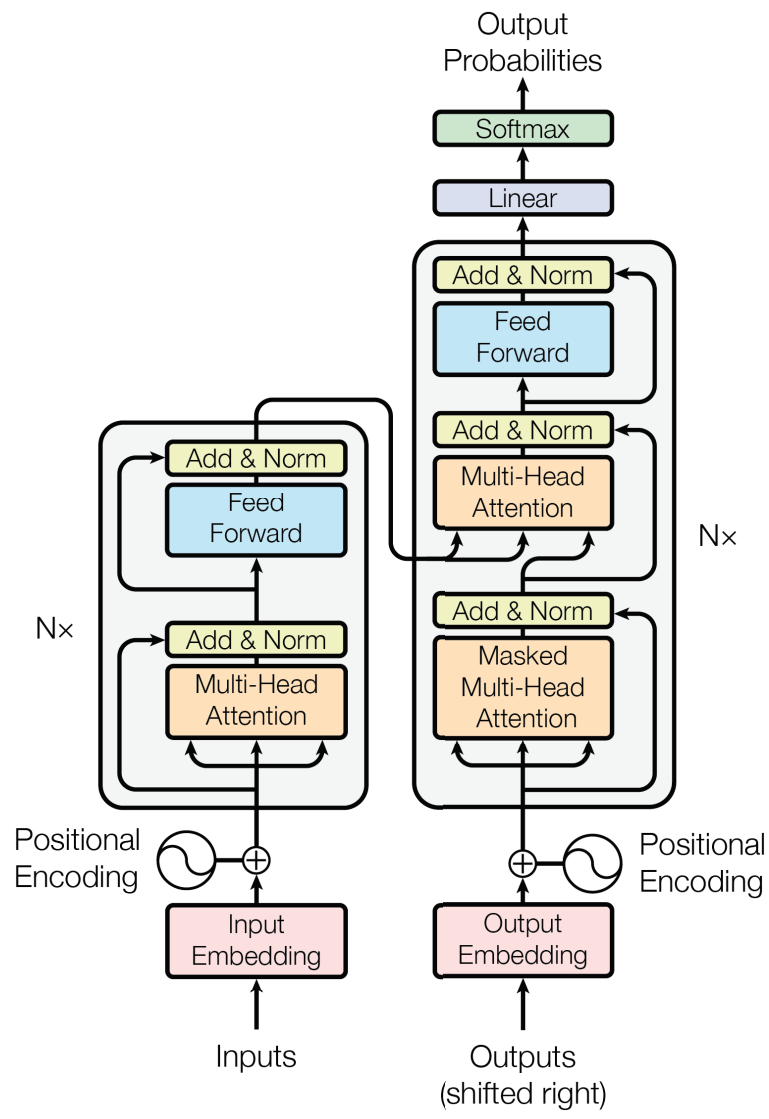

Figure 3.8: The first convolution-based sequence modeling approach proposed by Kaiser et al. [10]. 
fixed-length segments of a few hundred characters, and each segment is processed separately. As a result, the algorithm is not able to model dependencies that are longer than a fixed length. However, in tasks such as language modeling where the generated sentence can grow indefinitely, this behaviour is problematic.

To address these limitations, Dai et al. [23] proposed Transformer-XL. Transformer-XL consists of two techniques: a segment-level recurrence mechanism and a relative positional encoding scheme. During training, the representations computed for the previous segment are fixed and cached to be reused as an extended context when the model processes the next new segment. This additional connection increases the largest possible dependency length by $\mathrm{N}$ times, where $\mathrm{N}$ is the depth of the network, because contextual information is now able to flow across segment boundaries. Moreover, this recurrence mechanism also resolves the context fragmentation issue, providing necessary context for tokens in the front of a new segment.

Non-autoregressive methods use positional encodings to represent time among the input representations. Because with Transformer-XL we are applying segment-level recurrence, regural positional encodings do not work as they are not coherent when they are reused with the previous segments. To illustrate this issue, assume that we have a segment of four elements with positional encodings $[1,2,3,4]$. When we process the next in line segment, we will have the positions $[1,2,3,4,1,2,3,4]$ when the two segments are combined. Ideally, what we want is the positions to be $[1,2,3$, $4,5,6,7,8]$. To address this issues, Dai et al. [23] proposed the relative positional encoding scheme. Compared with the regular learnable positional embeddings, the relative positional encoding uses fixed embeddings with learnable transformations. 


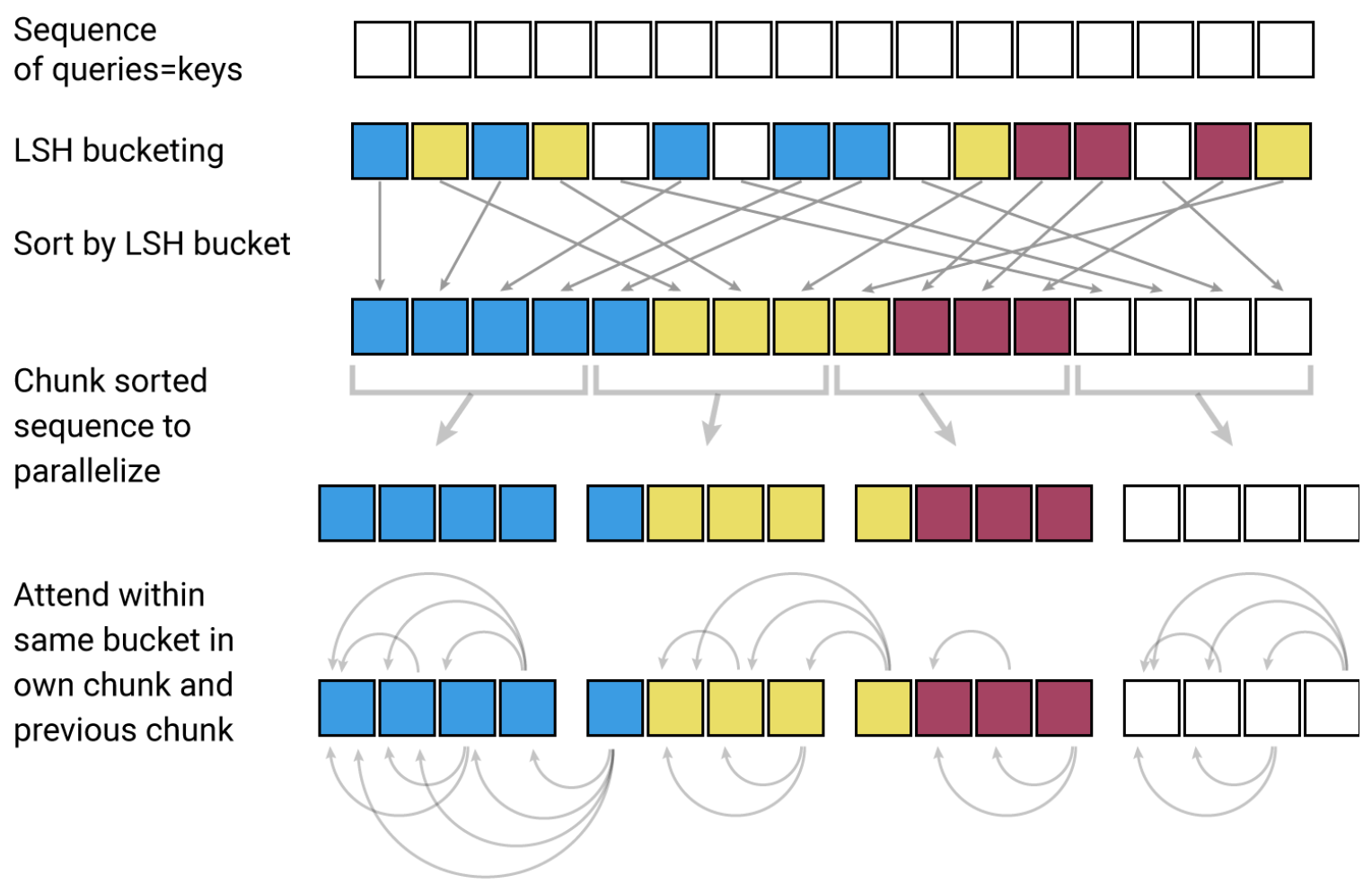

Figure 3.9: The process of Locality-sensitive-hashing that Reformer uses. Image taken from 14 .

\section{Reversible Transformer (Reformer)}

More recently, another sequence modeling approach has been proposed to mitigate the complexity of transformers. Reformer was proposed by Kitaev et al. [14] and introduced the locality-sensitive-hashing ( $\mathrm{LSH}$ ) to reduce the complexity of attending over long sequences. The challenge when applying a transformer model to a very large text sequence is handling the attention layer. LSH accomplishes this by computing a hash function that matches similar vectors together, instead of searching through all possible pairs of vectors. When the hashes are assigned, the sequence is rearranged to bring elements with the same hash together and divided into segments to enable parallel processing. Attention is then applied within these much shorter chunks. Figure 3.9 shows the process of reversible transformer. 


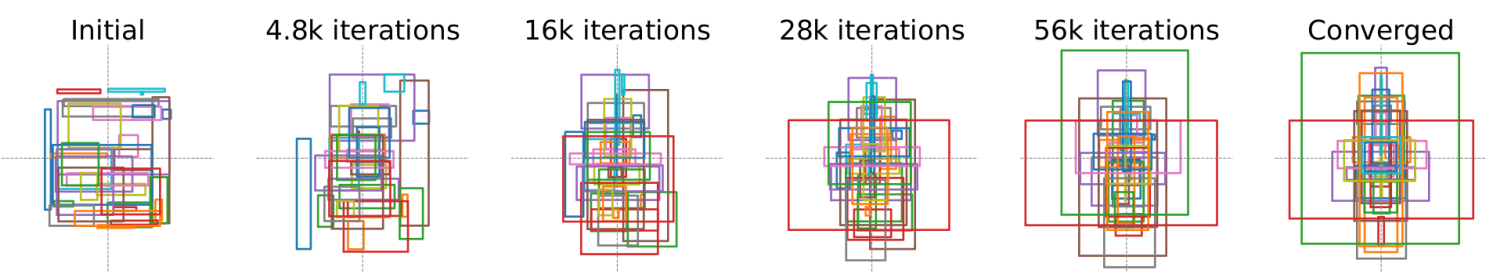

Figure 3.10: The progress of learning box coordinates of box convolutions. The figures was taken from $[15]$.

\subsection{Dynamically Sized Receptive Field}

Increasing the receptive field of a convolution layer without adding a computation overhead is a challenging task. By making deeper CNN models, we may be able to accumulate many fixed-sized receptive fields, however this comes at the cost of high computational demands. Nevertheless, this approach is shown to be successful in multiple state-of-the-art vision models [35, 36]. The overhead issue is often mitigated using a form of downsampling, either via pooling layers [37] or strided convolutions [38]. Yu et al. [5] proposed dilated convolutions, a method for enlarging the convolution kernel size by skipping intermediate pixels and thus, requiring less multadds operations.

\subsubsection{Deep Neural Networks with Box Convolutions}

The first work that suggested the use of learnable sized convolution kernels was box convolutions [15]. The idea of using box filters with summed-area tables [39], commonly known as integral images dates back many years and it is well-known to the Computer Vision community, as it became particularly popular with the work of Viola and Jones [40] in object detection. The summed-area table can be efficiently parallelized using the Parallel Prefix Sum method [41]. This operation can be further accelerated as a hardware functional unit dedicated to compute the multi-parameter prefix-sum operation 42 . 

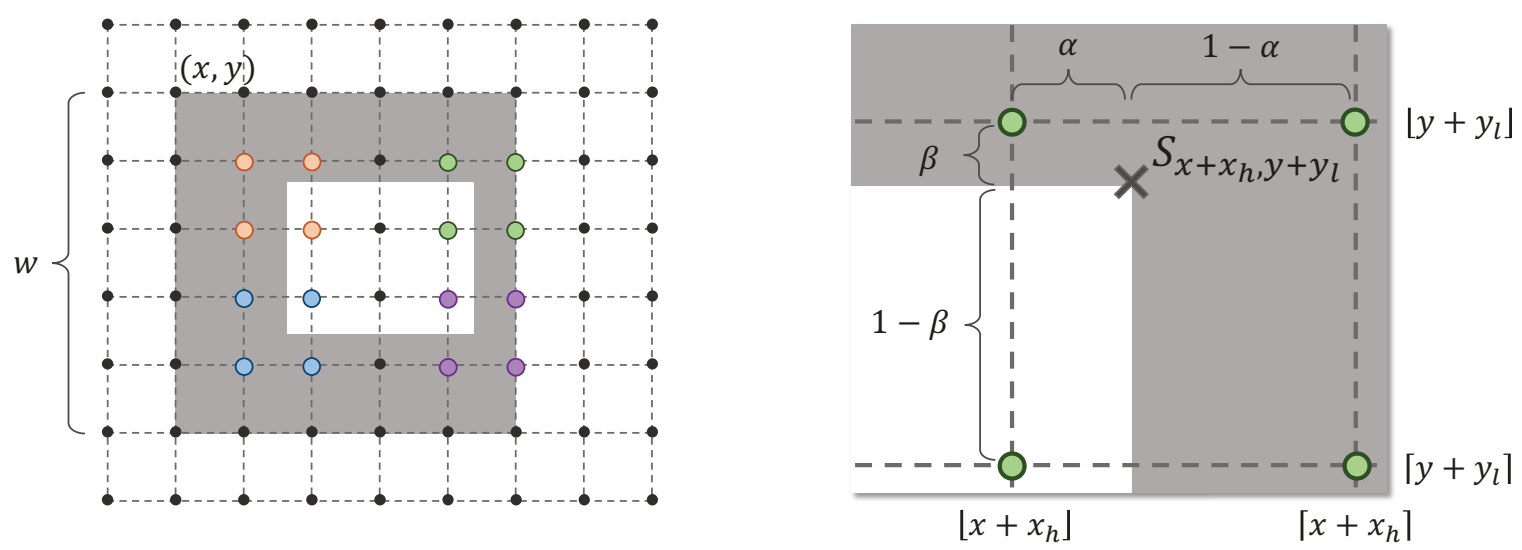

Figure 3.11: The interpolation approach for using real valued box coordinates as proposed by Zhang et al. [16].

The box convolution layer is a basic depthwise convolution but with special kernels called box kernels. A box kernel is a rectangular averaging filter. The idea is that instead of learning the kernel weights, the model learns the size and the offset of the filter. This process reduces the number of learnable parameters, and the computational efficiency achieved via the integral image trick. Figure 3.10 illustrates the kernels that a box convolution model learned over time.

\subsubsection{Large-Kernel Convolution Using Summed-Area Tables}

The box convolutions method is optimizing the kernel size parameters using approximate gradients by normalizing the sum by the area of the box. Zhang et al. [16] extended this idea by using interpolation to exploit non-integer coordinates. Figure 3.11 illustrates this interpolation approach. Inspired by this idea, we develop the proposed method for one-dimensional case of sequences. In contrast to the two previous methods, instead of using a fixed number of learnable sized kernels, we adaptively condition the size of the kernel on each input representation, effectively generating a different kernel size for each time-step token. 


\section{Chapter 4}

\section{Proposed Method}

\subsection{Introduction}

In this section, we present the proposed adaptive Time-aware Large Kernel (TaLK) convolution method. First, we will introduce the approach that computes a convolution operation using large kernels in $O(n)$ time, which assumes that left and right offsets are given. Next, we will present our proposed method for generating offsets dynamically for each time-step. We will then expand upon our method to use multiple heads and normalize the summed output vector. We also describe our proposed sequence modelling approach for decoding. Finally, we present the computational complexity analysis and comparison for the proposed method.

\subsection{Motivation}

Deep Learning models are the state-of-the-art in NLP, Speech Recognition, Computer Vision and many other fields. The remarkable deep learning results have been built on top of massive amounts of data and faster computation. Deploying these deep learning models is usually done either by serving the model on a cloud server, or deploying the model directly on the edge device. In both cases, the need for faster, less 
computationally and memory expensive networks is high. As we discussed in Chapter 3. the Transformer network [13] is currently considered the state-of-the-art method for modeling sequences. The success of the network lies on the attention mechanism that is employed between the input sequence tokens. Currently, attention is considered integral to achieve state-of-the-art results. Thus, all subsequent approaches utilize a form of attention. The major drawback of this attention mechanism is that it has quadratic time complexity $O\left(n^{2}\right)$ relevant to the sequence length. This is problematic, especially when we are interested in applying attention-based methods with long sequences. In this chapter, we are going to try to answer two questions:

- Can we replace attention and still maintain the state-of-the-art performance in various NLP tasks?

- Can we use a simpler and faster sequence modeling approach that is less computationally expensive compared to previous methods in the literature?

\subsection{One-dimensional Large Kernel Convolution}

When modeling sequences using attention, for each single time-step, we have to compute an attention distribution over all the available input representations. We multiply these scores with each vector representation and then sum all the re-scaled vectors together. This acts as the output representation for the current time-step. In this thesis, we argue that we do not need to compute the scaling (attention) factors for all time-steps. Instead, we propose that just summing the appropriate number of vector representations together (without attention scaling) is enough for the representation of a time-step.

Specifically, let $X=\left\{x_{1}, x_{2}, \ldots, x_{n}\right\}$ denote an input sequence, where $n$ is the 
length of the sequence, $x_{i} \in \mathbb{R}^{d}$ is the current input representation for the $i$-th word (i.e., the $i$-th time-step) and $d$ denotes the dimensionality of the vector representation (i.e., the number of channels).

For encoding the representation at the $i$-th time-step, we can express the proposed process by

$$
o_{i}=\sum_{j=\alpha_{i}^{l}}^{\alpha_{i}^{r}} x_{j}
$$

where $1 \leq \alpha_{i}^{l} \leq i \leq \alpha_{i}^{r} \leq n$ are the lower (left offset) and upper (right offset) bounds of the kernel size.

\subsubsection{Summed-area Table}

Equation 4.1 is simple but applying it for each time-step $i$ separately is not efficient. This is because we compute the same summations over the same values. Inspired by the work of Zhang et al. [16], we propose to use the summed-area table [39] to accelerate the summation process. Specifically, let $\mathcal{S}=\left\{\mathcal{S}_{0}, \mathcal{S}_{1}, \mathcal{S}_{2}, \ldots, \mathcal{S}_{n}\right\}$ be the summed-area table computed using

$$
\left\{\begin{array}{l}
\mathcal{S}_{0}=0 \\
\mathcal{S}_{i}=\mathcal{S}_{i-1}+x_{i}, \quad 1 \leq i \leq n .
\end{array}\right.
$$

Given the left offset $\alpha_{i}^{l}$ and the right offset $\alpha_{i}^{r}$, we can compute the summation denoted as $o_{i}$ of the features between these offsets using the summed-area table

$$
o_{i}=\mathcal{S}_{a_{i}^{r}}-\mathcal{S}_{a_{i}^{l}-1}
$$




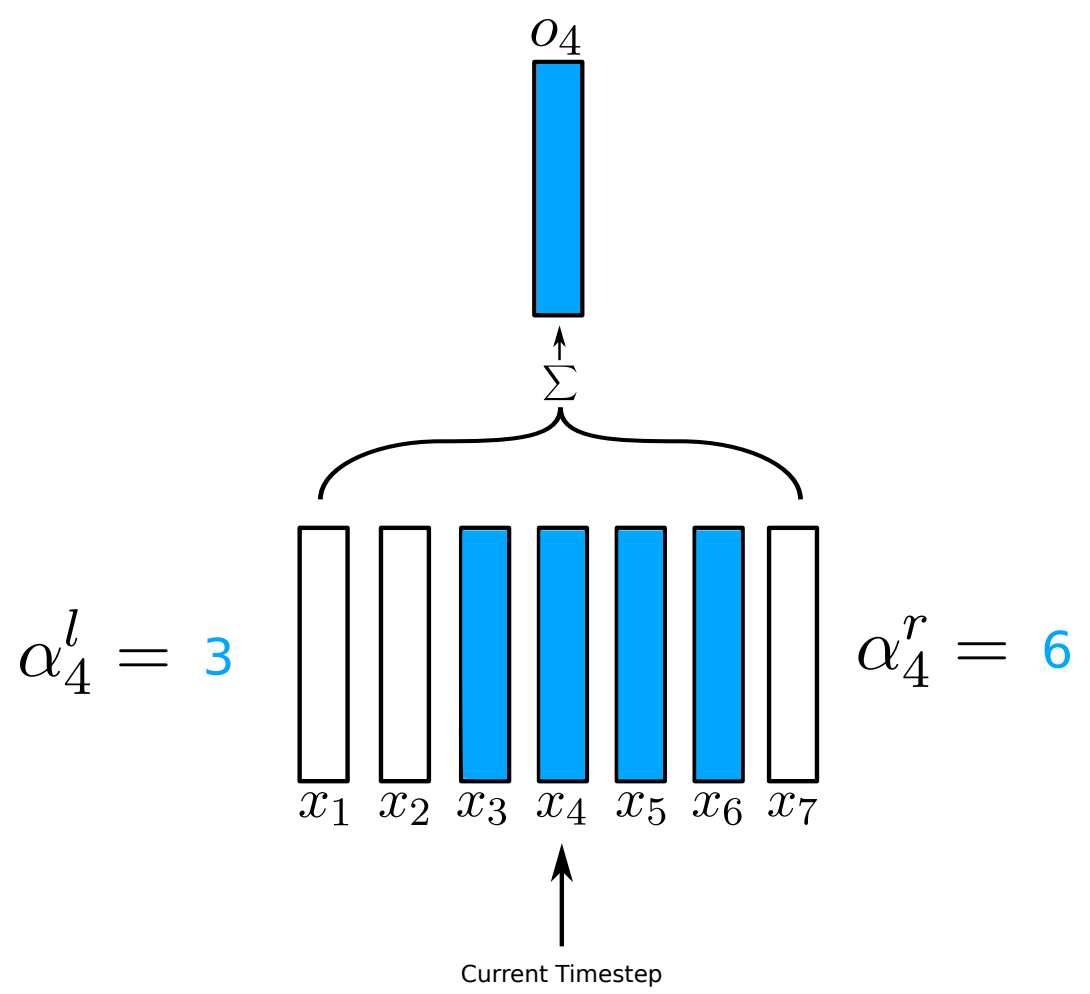

Figure 4.1: The One-dimensional Large Kernel Convolution operation. For the current time-step, given the left and right offsets, we sum all the representation vectors inside these boundaries.

We call the process of computing the summed-area table and applying a given set of left and right offsets as the One-dimensional Large Kernel Convolution operation. The summed-area table is computed only once and can be reused to compute any summation between two time-steps. Figure 4.1 illustrates the One-dimensional Large Kernel Convolution operation.

\subsection{Time-aware Large Kernel Generation}

Given the one-dimensional large kernel convolution above, it is important to determine the left and right offsets for computing representations at each time-step. The key of the proposed method is an adaptive time-aware large kernel convolution operation which has kernel sizes that vary over time as a learned function of the 
individual time steps; that is, we propose to learn the offsets of the summation kernel above for each time-step.

Specifically, we propose to use a function $f^{\{l, r\}}: \mathbb{R}^{d} \rightarrow \mathbb{R}$ to generate for each $x_{i}$ the left $\tilde{a}_{i}^{l}$ and right $\tilde{a}_{i}^{r}$ relative offsets, where $\tilde{a}_{i}^{\{l, r\}}=\sigma\left(f^{\{l, r\}}\left(x_{i}\right)\right) \in[0,1]$. For each $\tilde{a}_{i}^{\{l, r\}}$ relative offset, we convert it to the absolute offset counterpart in the following way

$$
\begin{aligned}
& a_{i}^{l}=i-\tilde{a}_{i}^{l} \cdot l_{\max } \\
& a_{i}^{r}=i+\tilde{a}_{i}^{r} \cdot r_{\max },
\end{aligned}
$$

where $l_{\max } \in \mathbb{Z}_{\geq 0}$ is the maximum allowed tokens to the left and $r_{\max } \in \mathbb{Z}_{\geq 0}$ is the maximum allowed tokens to the right.

The absolute offsets up to this point represent real positive numbers. In the next step, we need to convert these numbers to integer indexes so we can select from the summed-area table using the Equation (4.3). Inspired by Zhang et al. [16], we use one-dimensional interpolation to sample from the summed-area table by using the positive real-valued offsets $a_{i}^{l}, a_{i}^{r}$ as follows

$$
\begin{aligned}
\mathcal{S}_{a_{i}^{l}-1} & =\gamma^{l} \cdot \mathcal{S}_{\left\lfloor a_{i}^{l}\right\rfloor-1}+\left(1-\gamma^{l}\right) \cdot \mathcal{S}_{\left\lceil a_{i}^{l}\right\rceil-1}, \\
\mathcal{S}_{a_{i}^{r}} & =\left(1-\gamma^{r}\right) \cdot \mathcal{S}_{\left\lfloor a_{i}^{r}\right\rfloor}+\gamma^{r} \cdot \mathcal{S}_{\left\lceil a_{i}^{r}\right\rceil},
\end{aligned}
$$

where $\lfloor$.$\rfloor and \lceil$.$\rceil are the floor and ceiling operators, \gamma^{l}=\left\lceil a_{i}^{l}\right\rceil-a_{i}^{l}$ and $\gamma^{r}=a_{i}^{r}-\left\lfloor a_{i}^{r}\right\rfloor$. The above equation is continuous and differentiable in the interpolation neighborhood. 
The partial derivatives of $\mathcal{S}_{a_{i}^{\{l, r\}}}$ with respect to $\tilde{a}_{i}^{\{l, r\}}$ are given by

$$
\begin{aligned}
\frac{\partial \mathcal{S}_{a_{i}^{l}-1}}{\partial \tilde{a}_{i}^{l}} & =l_{\max }\left(\mathcal{S}_{\left\lfloor a_{i}^{l}\right\rfloor-1}-\mathcal{S}_{\left\lceil a_{i}^{l}\right\rceil-1}\right), \\
\frac{\partial \mathcal{S}_{a_{i}^{r}}}{\partial \tilde{a}_{i}^{r}} & =r_{\max }\left(\mathcal{S}_{\left\lceil a_{i}^{r}\right\rceil}-\mathcal{S}_{\left\lfloor a_{i}^{r}\right\rfloor}\right) .
\end{aligned}
$$

The partial derivatives of $\mathcal{S}_{a_{i}^{\{l, r\}}}$ with respect to $\mathcal{S}_{\left\lfloor a_{i}^{\{l, r\}}\right\rfloor}$ and $\mathcal{S}_{\left\lceil a_{i}^{\{l, r\}}\right\rceil}$ tokens are given by

$$
\begin{aligned}
& \frac{\partial \mathcal{S}_{a_{i}^{l}-1}}{\partial \mathcal{S}_{\left\lfloor a_{i}^{l}\right\rfloor-1}}=\gamma^{l}, \quad \frac{\partial \mathcal{S}_{a_{i}^{l}-1}}{\partial \mathcal{S}_{\left\lceil a_{i}^{l}\right\rceil-1}}=\left(1-\gamma^{l}\right), \\
& \frac{\partial \mathcal{S}_{a_{i}^{r}}}{\partial \mathcal{S}_{\left\lfloor a_{i}^{r}\right\rfloor}}=\left(1-\gamma^{r}\right), \quad \frac{\partial \mathcal{S}_{a_{i}^{r}}}{\partial \mathcal{S}_{\left\lceil a_{i}^{r}\right\rceil}}=\gamma^{r} .
\end{aligned}
$$

\subsection{Output Normalization and Offsets Dropout}

The idea of summing all the features in a window of size $\left[a_{i}^{l}, a_{i}^{r}\right]$ works well for shallow models. However, as the representation vectors at different time-steps are computed from summations over different numbers of neighbors, their magnitudes of values can be different. As we introduce more layers, the disproportional magnitude of the inputs makes learning harder for the nodes in the layers that follow. To address this problem, we propose to normalize the output representations of TaLK Convolutions as follows

$$
\tilde{o}_{i}=o_{i} \cdot\left(\frac{1}{l_{\max }+r_{\max }+1}\right) .
$$

Such a simple window size based normalization can effectively get rid of the output magnitude differentiation problem resulted from summation kernels.

In addition, we regularize the predicted offsets $\tilde{a}_{i}^{\{l, r\}}$ using Dropout 43, 44. Specifically, during training we drop out every predicted offset with probability $p$. This helps to prevent the model from quickly optimizing towards a specific window size and be 
able to generate more diverse offsets.

\subsection{Multi-headed Kernels}

Although the offset computation above provides a mechanism that offers adaptive receptive fields for summation kernels at different time steps, a single pair of left and right offsets for all $d$ dimensions cannot yield good results, as different features might be related to their counterpart in the neighbor tokens in different way. Inspired by the idea of multi-head attention [12, 13], we further propose to extend our proposed convolution kernel into a multi-head version by allowing different representation features, i.e., channels, to have different left and right offsets for each time-step. Moreover, instead of having entirely different convolution offsets across multiple channels, we adopt a depthwise version by separating the feature channels into multiple groups, each of which share the same pair of left and right offsets.

Specifically, we tie every subsequent number of $R=\frac{d}{H}$ channels together and group the channels into $H$ groups for each $x_{i}$, where $H$ is the number of heads. This results to $\hat{X}=\left\{\hat{x}_{1}, \hat{x}_{2}, \ldots, \hat{x}_{n}\right\}$, where $\hat{x}_{i} \in \mathbb{R}^{H \times R}$. Then we use a function $f^{\{l, r\}}$ : $\mathbb{R}^{H \times R} \rightarrow \mathbb{R}^{H}$ to generate for each $\hat{x}_{i}$ a vector of $H$ left relative offsets $\tilde{\alpha}_{i}^{l}$ or right

relative offsets $\tilde{\alpha}_{i}^{r}$ via $\tilde{\alpha}_{i}^{\{l, r\}}=\sigma\left(f^{\{l, r\}}\left(\hat{x}_{i}\right)\right) \in[0,1]^{H}$. Figure 4.2 illustrates the Timeaware Large Kernel Convolution operation for a specific time-step during encoding using 2-headed kernels.

\subsection{Decoding Using TaLK Convolutions}

In an encoder/decoder sequence generation scheme [8], the encoder part of the model has access to both past and future tokens. The decoding part, however, must have 


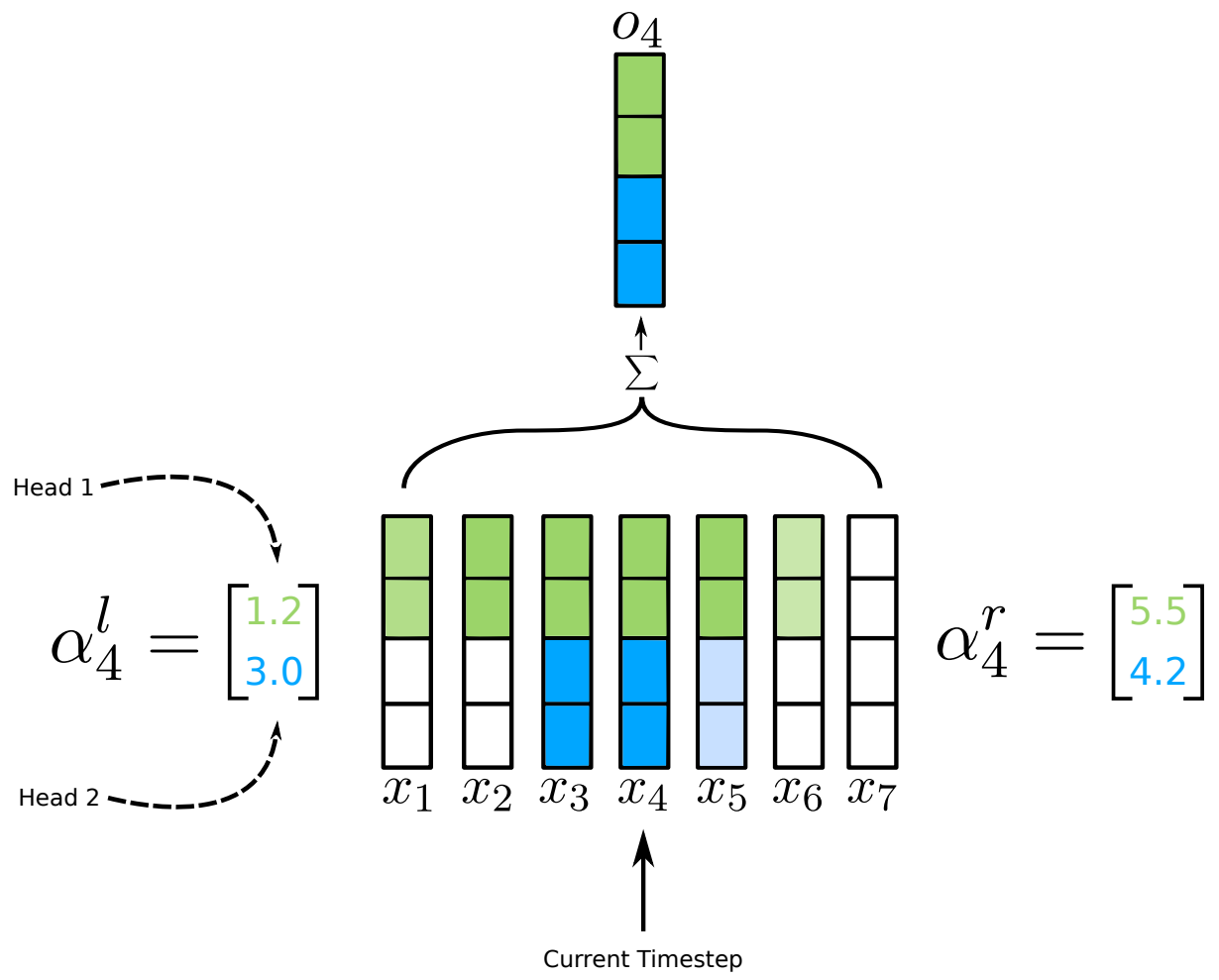

Figure 4.2: The Time-aware Large Kernel convolution operation. For the current time-step, we compute the left and right offsets for each head, and then sum all the representation vectors inside these boundaries. This operation can be efficiently computed using summed-area tables with time complexity $O(\log (n))$ and compute the output representation for each time-step in $O(n)$ time. 


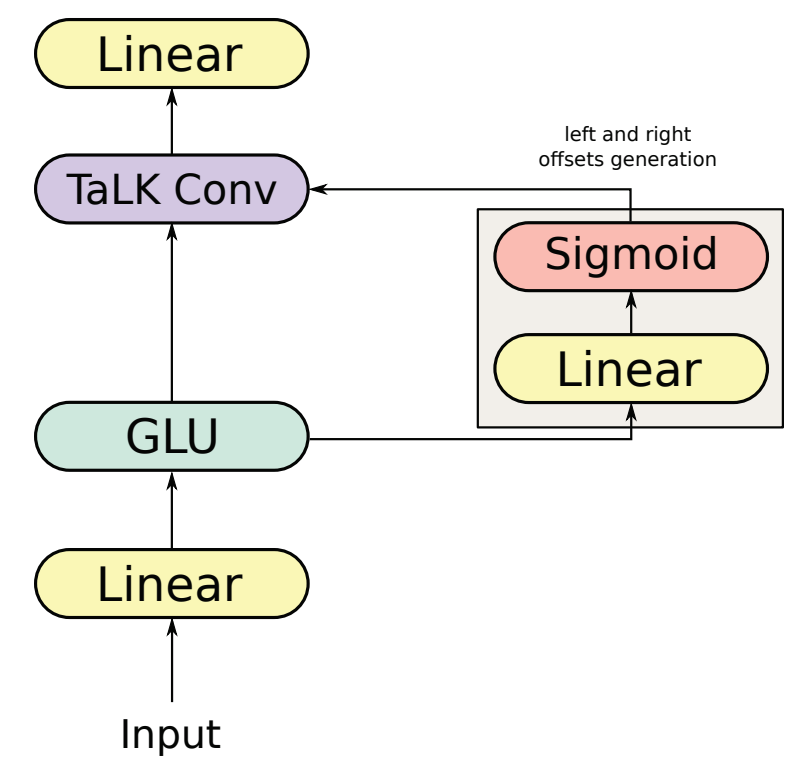

Figure 4.3: The architecture of the proposed TaLK Convolution unit.

access only to past tokens that are generated so far. Enforcing this with TaLK Convolutions is straightforward by setting the $r_{\max }$ value to zero.

\subsection{Module Architecture and Implementation}

For sequence modeling, we follow a similar module architecture as described in 12 . Specifically, we apply a linear layer to project the input embedding tokens from $d$ to $2 d$ and then we apply a gated linear unit (GLU) [45. Next, we apply the TaLK Convolution operation as described in Section 4.4. Finally, we apply a projection layer to the output representations from TaLK Convolution with size $W \in \mathbb{R}^{d \times d}$. Figure 4.3 illustrates the proposed TaLK Convolution unit. We substitute all ReLU activation functions with the Swish function [46] which we found empirically to yield higher performance. The Swish activation function is defined as

$$
f(x)=x \cdot \sigma(x)
$$




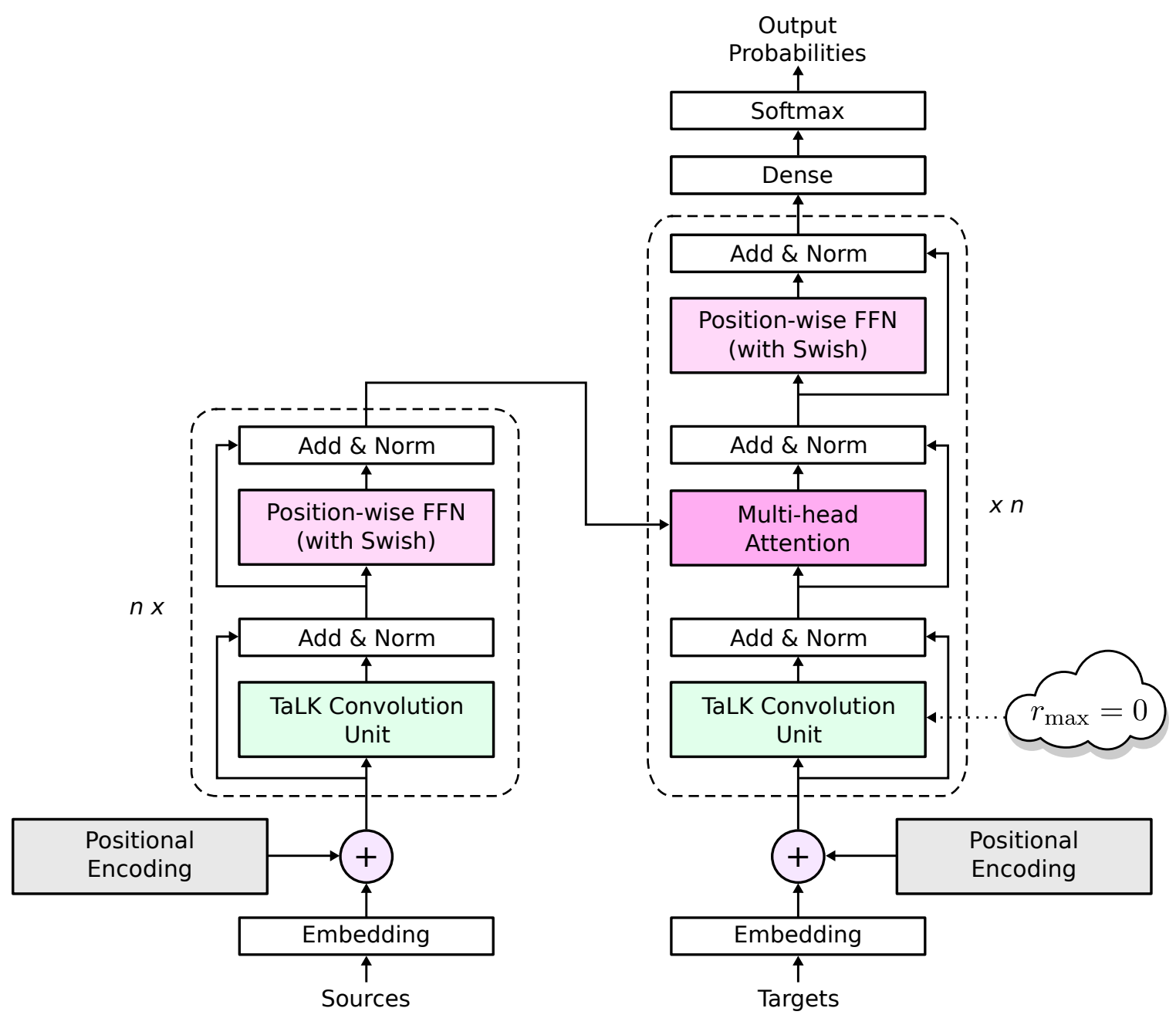

Figure 4.4: The model architecture of the proposed TaLK Convolution network.

The overall model architecture used for the TaLK Convolution network is illustrated in Figure 4.4 .

The summed-area table (Equation 4.2) can be efficiently computed on a GPU by performing a fast Parallel Prefix Sum [41] over the token dimension. This operation is usually efficiently implemented on modern deep learning frameworks (e.g. PyTorch 1 and Tensorflow ${ }^{2}$ ) under the name of cumulative sum. Applying the relative offsets

1 https://pytorch.org/

2 https://www.tensorflow.org/ 
Table 4.1: Maximum path lengths, per-layer complexity and minimum number of sequential operations for different layer types. $n$ is the sequence length, $d$ is the representation dimension and $k$ is the kernel size of convolutions.

\begin{tabular}{lccc}
\hline Layer Type & Complexity per Layer & $\begin{array}{c}\text { Sequential } \\
\text { Operations }\end{array}$ & Maximum Path Length \\
\hline Recurrent 8 & $O\left(n \cdot d^{2}\right)$ & $O(n)$ & $O(n)$ \\
Convolutional & $O\left(k \cdot n \cdot d^{2}\right)$ & $O(1)$ & $O\left(\log _{k}(n)\right)$ or $O(n / k)$ \\
11,21 & $O\left(n^{2} \cdot d\right)$ & $O(1)$ & $O(1)$ \\
Self-Attention 13 & $O(k \cdot n \cdot d)$ & $O(1)$ & $O(n / k)$ \\
Dynamic Convolutions 12 & $O(n \cdot d)$ & $O(\log (n))$ & $O\left(n /\left(l_{\max }+r_{\max }+1\right)\right)$ \\
\hline
\end{tabular}

to the summed-area table using core functions from deep learning frameworks is not a trivial task. Such an implementation is usually very inefficient leading to slower computation and memory overhead. For this reason, we implemented the operation using CUDA kernels that enabled us to parallelize the computation for each token. Chapter $\mathrm{A}$ in the Appendix includes the CUDA pseudocode implementation.

\subsection{Computational Complexity}

In this section, we compare the complexity of the TaLK Convolution operation against different modules for encoding an input sequence of representations. This comparison is shown on Table 4.1. We follow a similar comparison as analyzed by Vaswani et al. [13]. Our comparison is based on three criteria: the time complexity of the operation, the amount of computations that can be executed in parallel and the path length between long-range dependencies.

As shown in Table 4.1, our proposed method requires the least number of 
operations. Specifically, it has a linear time complexity to encode a sequence and does not depend on hyper-parameter decisions such as the kernel size. In terms of the number of computations that can be parallelized, our method needs logarithmic time to compute the summed-area table (Equation 4.2). This is because we consider the fast Parallel Prefix Sum algorithm (Ladner and Fischer [41]) which has $O(\log (n))$ complexity to compute the summed-area table. It is true that our method does not have a constant number of sequentially executed operations like all the other non-autoregressive counterpart methods, but the logarithmic time our method is requiring is inexpensive to compute even for very long sequences.

It is shown by Kolen et al. 47] that a short path between any combination of token positions in the input and output sequences makes it easier to learn long-range dependencies. In practice, doubts have been cast over the ability of self-attention to model long-range dependencies [12,48]. Wu et al. [12 showed that using a limited context window can outperform self-attention. Our method has the advantage that the number of required computations is independent of the maximum window size and thus, it can be tuned or learned without extra cost.

\subsection{Conclusion}

In this chapter, we discussed the motivation of why we need to investigate alternative methods to attention and how this can speed up the inference of the deployed deep learning solutions. We introduced the one-dimensional large kernel convolution using the summed-area tables and we described how to make this process adaptive to the input sequences. We presented extensions to the base operation in order to further improve the performance. Finally, we compared our method's time complexity with the other methods in the literature. In the next chapter, our experiments and model 
evaluation methods will be introduced. 


\section{Chapter 5}

\section{Experiments and Evaluation}

\section{$5.1 \quad$ Introduction}

In this chapter, we present the empirical experimentation details that we followed in order to evaluate the proposed TaLK Convolution sequence modeling method. We decided to evaluate our method using four different natural language processing tasks: Machine Translation (Section 5.2), Language Modeling (Section 5.3), Abstractive Summarization (Section 5.4) and Sentence Classification (Section 5.5). We used six different standard benchmark datasets across these four tasks. In Section 5.6, we conduct an ablation study and show that each proposed extension to the basic idea helps to achieve better results. Finally, in Section 5.7, we compared the inference speed between our method and two of the state-of-the-art methods in the literature.

\subsection{Machine Translation}

Machine translation is the touchstone task that sequence modeling methods are evaluated with. It involves the use of mathematical and algorithmic techniques to translate documents from one language to another. Performing effective translation is considerably challenging even for humans, requiring proficiency in areas such as morphology, 
Table 5.1: Machine translation benchmark datasets statistics.

\begin{tabular}{l|ccc|c}
\hline \multirow{2}{*}{ Dataset } & \multicolumn{3}{|c|}{ No. of Examples } & \multirow{2}{*}{ Max Sentence Length } \\
\cline { 2 - 4 } & Training & Validation & Testing & \\
\hline WMT En-Fr & $35,762,532$ & 26,854 & 3,003 & 251 \\
WMT En-De & $4,500,966$ & 3,000 & 3,003 & 338 \\
IWSLT De-En & 160,239 & 7,283 & 6,750 & 245 \\
\hline
\end{tabular}

syntax, and semantics, as well as a solid understanding of cultural sensitivities, for both languages, and in extention the associated societies under consideration.

\subsubsection{Datasets}

For this task, we decided to report results on three mainstream benchmark datasets: WMT English to German (En-De), WMT English to French (En-Fr) and IWSLT German to English (De-En). We decided that these benchmark datasets are good representatives of high-resource, medium-resource and low-resource language pairs. Table 5.1 shows the number of samples each benchmark dataset contains.

\section{WMT English to French (En-Fr)}

The WMT 2014 En-Fr language pair dataset contains approximately 36M training sentence pairs from WMT'14 $4^{1}$ translation competition. We used Byte Pair Encoding (BPE) 25] tokenization to extract a common vocabulary between the two languages. Similar to $\mathrm{Wu}$ et al. [12], we set the size of the vocabulary to 40,000 subword tokens. The validation dataset is composed by the newstest2012 and newstest2013 testing sets and we report results on the newstest2014.

\footnotetext{
1 https://www.statmt.org/wmt14/translation-task.html
} 


\section{WMT English to German (En-De)}

For the WMT English to German (En-De), we use the same setup as 13. The dataset is based on WMT'16 training data ${ }^{2}$ and contains approximately $4.5 \mathrm{M}$ pairs. We extracted a shared vocabulary between English and German using Byte Pair Encoding of size 32,000 subword tokens. We evaluate the proposed method using the newstest2013 as the validation dataset and the newstest2014 as the testing dataset.

\section{IWSLT German to English (De-En)}

For the IWSLT German to English (De-En), we pre-process the IWSLT 2015 3 benchmark dataset following Edunov et al. [49] instructions. The dataset consists of about 160,000 training sentence pairs. Using Byte Pair Encoding, we extracted a vocabulary of size 10,000 subword tokens. For this benchmark only, all sentences are lowercased following $\mathrm{Wu}$ et al. [12] setup.

\section{Evaluation Metrics}

For all language pairs, we measure case-sensitive BLEU score [50 using the mutli-blev ${ }^{4}$ script. In addition, we apply compound splitting with the WMT En-De benchmark dataset similar to Vaswani et al. 13.

We trained five random initializations of a each model configuration and reported test accuracy of the seed which resulted in the highest validation BLEU. For all datasets we used beam search to generate translated sentences. We found empirically that for WMT En-De a beam width of 4 works the best. Similarly, for IWSLT De-En we used a beam width of 5 and for WMT En-Fr a beam width of 6 . Following Wu

\footnotetext{
2 http://www.statmt.org/wmt16/translation-task.html

3 http://workshop2015.iwslt.org/index.php

${ }_{4}^{4}$ https://github.com/moses-smt/mosesdecoder/blob/master/scripts/generic/ multi-bleu.perl
} 
et al. [12], we tuned the length generation penalty on the validation set. In addition, similar to Vaswani et al. [13] we averaged a number of checkpoints. This number was tuned on the validation set. This technique is shown to improve the overall performance of the model.

\subsubsection{Experiment Details}

In this section, we describe the details of the experiments like the hyper-parameters the model were trained with, the optimization method, as well the hardware details in order to ensure that our results are reproducible.

\section{Hyper-Parameters}

In order for our method to be comparable with the competitive methods described in Vaswani et al. [13] and Wu et al. [12], we closely followed their model configurations. Specifically, similar to Wu's et al. [12] Big Model setup, the model's hidden size $d$ was set to 1024 and the feed-forward hidden size $d_{\mathrm{ff}}$ was set to 4096 . In addition, the number of layers for the encoder and the decoder was set to 7 and 6 respectively and the number of heads was set to 16 . The proposed method introduced two new hyper-parameters, the maximum allowed tokens to the left $l_{\max }$ and the maximum allowed tokens to the right $r_{\max }$. Each layer in the encoder and the decoder can have their own value for these two hyper-parameters. For the encoder model, we set the $l_{\max }$ and $r_{\max }$ values to 3,7,15 for the first three layers and 31 for the last

four layers. For the decoder, we set the $l_{\max }$ to $3,7,15,31 \times 3$ for each layer and the hyper-parameter $r_{\max }$ to zero since we do not want the decoder to have access to future tokens.

For IWSLT De-En, the model's hidden size $d$ was set to 512, the feed-forward hidden size $d_{\mathrm{ff}}$ was set to 1024 and the number of layers for the encoder and the 
decoder was set to 7 and 6 respectively. The number of heads was set to 4 and the $l_{\max }, r_{\max }$ values to $1,3,7,15 \times 4$ for each layer.

\section{Optimization}

We followed the same optimization settings as $\mathrm{Wu}$ et al. [12]. We used the Adam optimizer [51] with default values. In addition, our models were optimized using the cosine learning rate schedule [52]. We linearly warmed up for $10 \mathrm{~K}$ steps from $10^{-7}$ to $10^{-3}$. Figure 5.1 visualize the learning rate schedule that is used. For IWSLT De-En, we used the inverse square root learning rate schedule [13] with linear warm up for 4,000 steps and maximum learning rate 0.0005 . Figure 5.2 graphically illustrates the learning rate trajectory. We set the dropout to 0.3 for WMT En-De and IWSLT De-En and 0.1 for WMT En-Fr.

Instead of batching samples based on a fixed number of examples in the batch, we batched samples based on the maximum number of tokens allowed in the batch. This helps us avoid having multiple unnecessary padding tokens by batching together sentences that have similar length. This batching strategy expedites the training process significantly. The batch size was set to 3,584 tokens per batch for WMT En-De and WMT En-Fr datasets.

We trained our models using 8 GPUs for WMT En-De and WMT En-Fr benchmarks. Distributed training helps increase the batch size by running the same version of the model in parallel using multiple batches and then updates the model using the averaged gradient updates from all parallel nodes. In addition, in order to further increase the batch size, we accumulated the gradients for 16 batches before applying an update which results in an effective batch size of $3,584 * 8 * 16 \approx 450 \mathrm{~K}$ 


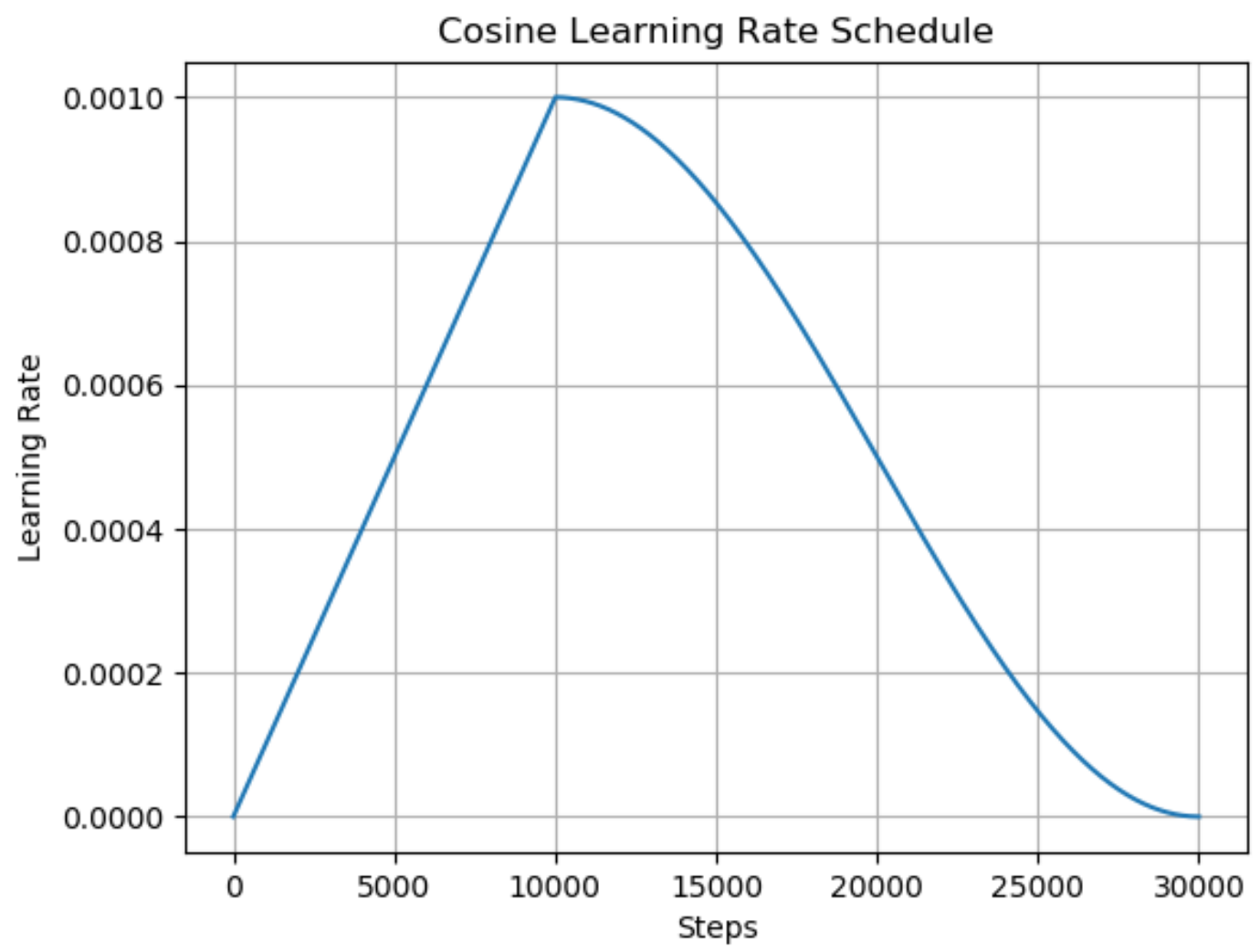

Figure 5.1: The cosine learning rate scheduler used with WMT En-Fr and WMT En-De bechmark datasets. 


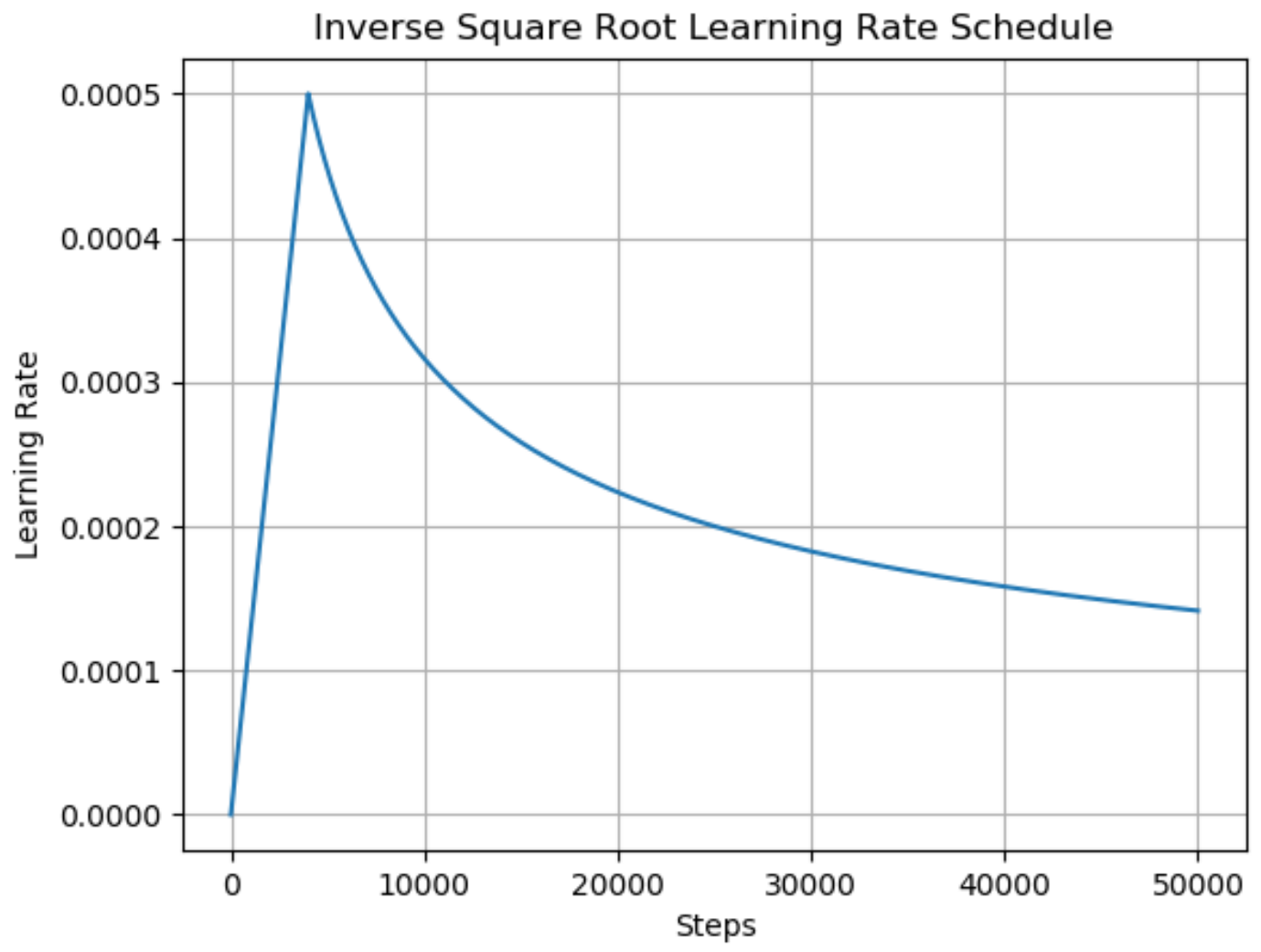

Figure 5.2: The inverse square root learning rate scheduler used with the IWSLT De-En bechmark dataset. 
tokens. We aimed for a high batch size because Popel et al. [53] showed that the nonautoregressive methods can yield better results with a high batch size. We trained the WMT En-De model for 30K steps and the WMT En-Fr for 80K steps. For IWSLT De-En, we trained on a single GPU with 4,000 maximum tokens per batch for 50K steps.

\section{Hardware Details}

We trained the WMT En-De and WMT En-Fr models on 8 NVIDIA RTX 2080 Ti GPUs using mixed-precision training [54]. The simultaneous use of 16-bit and 32-bit floating-point types in a model during the training phase, as in mixed precision, allows for both a faster runtime and the use of less memory. The numeric stability keeping certain parts of the model in the 32-bit model allows for a lower iteration time while not sacrificing the training quality in terms of the standard evaluation metrics.

We employed our own CUDA implementation, wrapped as a standalone PyTorch layer for the TaLK Convolution operation. Chapter $\mathrm{A}$ in the Appendix describes the pseudo-code that was developed for the CUDA implementation. All experiments were run using Fairsed ${ }^{5}$ toolkit.

\subsubsection{Results}

We demonstrate the effectiveness of our model in the WMT En-Fr and WMT En-De translation benchmarks. Table 5.2 shows the BLEU score that our method achieves on WMT En-Fr against other state-of-the-art (SoTA) methods in the literature. This benchmark is considered indicative for the effectiveness of a sequence learning method. This is because it contains a large number of training examples (36M)

\footnotetext{
$\sqrt[5]{\text { https://github.com/pytorch/fairseq }}$
} 
Table 5.2: Machine translation accuracy in terms of BLEU for WMT En-Fr on newstest2014.

\begin{tabular}{lcr}
\hline Model & Param (En-Fr) & WMT En-Fr \\
\hline ConvS2S 11 & $225 \mathrm{M}$ & 40.5 \\
Transformer 13$]$ & $222 \mathrm{M}$ & 41.0 \\
Weighted Transformer 55 & $222 \mathrm{M}$ & 41.4 \\
RNMT+ 56 & $391 \mathrm{M}$ & 41.0 \\
Transformer + Relative Position 57 & - & 41.5 \\
Large-scale Transformer $[58$ & $222 \mathrm{M}$ & $\mathbf{4 3 . 2}$ \\
DynamicConv 12$]$ & $222 \mathrm{M}$ & $\mathbf{4 3 . 2}$ \\
\hline TaLK Convolution (Ours) & $221 \mathrm{M}$ & $\mathbf{4 3 . 2}$ \\
\hline
\end{tabular}

which shows the generalization capabilities of a method. The proposed method, TaLK Convolutions is able to match the state-of-the-art 43.2 BLEU score. This shows that the proposed method is able to achieve SoTA results without using the expensive attention operation.

Additionally for WMT En-De, our method is only 0.1 BLEU points behind the current state-of-the-art score. This difference is not significant. This specific dataset is heavily criticized as a challenging dataset to reach higher results without augmenting the dataset either using new data samples or use back-translation techniques. It is also important to underline, however, that our method uses the least number of parameters compared to the other counterpart methods.

Furthermore, we report results for the IWSLT De-En benchmark dataset. This dataset presents a problem where the model should be able to learn from a very limited number of translation pair samples. A model should be able to generate convincing 
Table 5.3: Machine translation accuracy in terms of BLEU for WMT En-De on newstest2014.

\begin{tabular}{lcr}
\hline Model & Param (En-De) & WMT En-De \\
\hline ConvS2S 11 & $216 \mathrm{M}$ & 25.2 \\
Transformer 13 & $213 \mathrm{M}$ & 28.4 \\
Weighted Transformer 55 & $213 \mathrm{M}$ & 28.9 \\
RNMT+ $56 \mid$ & $379 \mathrm{M}$ & 28.5 \\
Transformer + Relative Position 57 & - & 29.2 \\
Large-scale Transformer 58 & $210 \mathrm{M}$ & 29.3 \\
DynamicConv 12 & $213 \mathrm{M}$ & $\mathbf{2 9 . 7}$ \\
Reformer 14$]$ & $213 \mathrm{M}$ & 29.1 \\
\hline TaLK Convolution (Ours) & $209 \mathrm{M}$ & 29.6 \\
\hline
\end{tabular}

translations despite the few number of training examples. Table 5.4 presents the performance our method achieves against other methods in the literature. Specifically, TaLK Convolution is able to achieve state-of-the-art results using the least amount of trainable parameters. This is very encouraging for the effectiveness of our method as it shows that attention can be replaced without compromising the performance of the model. Additionally, we gain inference speed which we will describe in details in Section 5.7.

\subsection{Language Modeling}

Perhaps the most fundamental task in natural language processing is that of language modeling. Language modeling (LM) is an essential piece of almost any application of NLP either explicitly (e.g. BERT [60] applications) or implicitly (e.g. a translation model is a conditional language model). Language modeling can be described as the 
Table 5.4: Machine translation accuracy in terms of BLEU on IWSLT De-En.

\begin{tabular}{lcr}
\hline Model & Param & IWSLT De-En \\
\hline Variational Attention $[59]$ & - & 33.1 \\
Transformer $[13$ & $47 \mathrm{M}$ & 34.4 \\
DynamicConv [12 & $43 \mathrm{M}$ & 35.2 \\
\hline TaLK Convolution (Ours) & $42 \mathrm{M}$ & $\mathbf{3 5 . 5}$ \\
\hline
\end{tabular}

process of creating a model to predict words or linguistic components given previous words or components. A language model should be able to implicitly capture syntactic and semantic relationships among words or components. These models have applications to tasks such as machine translation and text summarization which can help to generate more relevant, human-sounding sentences.

\subsubsection{Datasets}

We experimented on the WikiText-103 benchmark dataset. This a relatively new dataset release in 2016 by Merity et al. 61]. The WikiText language modeling dataset is a collection of over 100 million tokens extracted from the set of verified Good and Featured articles on Wikipedia. The dataset has a very large vocabulary and retains the original case, punctuation and numbers in the sentences. This is considered a standard dataset for assessing the long term dependence capabilities of sequence models.

Table 5.5 contains some statistics about the dataset. The training data contains approximately $100 \mathrm{M}$ tokens. A vocabulary of about $260 \mathrm{~K}$ tokens was used, by discarding all tokens with a frequency below 3 as described in [61]. We learn a byte-pair 
Table 5.5: WikiText-103 benchmark dataset for language modeling.

\begin{tabular}{l|ccc}
\hline & Train & Validation & Test \\
\hline Articles & 28,475 & 60 & 60 \\
Tokens & $103,227,021$ & 217,646 & 245,569 \\
Vocabulary Size & & 267,735 & \\
OoV & & $0.4 \%$ & \\
\hline
\end{tabular}

encoding (BPE) of $32 \mathrm{~K}$ codes on the training data which results to a vocabulary of 33,337 tokens. We followed Baevski and Auli et al. [1] and applied adaptive input representations. Adaptive input embeddings extend the adaptive softmax to input word representations. Figure 5.3 illustrates this process. Token embeddings are clustered into groups based on their frequency and each cluster has its own dimensionality followed by a linear projection for each cluster to bring the representations into a common dimensionality. We replicated their setup and partition the training data into blocks of 512 contiguous tokens while ignoring document boundaries.

\subsubsection{Experiment Details}

In this section, we describe the details of the experiments such the hyper-parameters the model were trained with, the optimization method as well the hardware details in order to ensure that our results are reproducible.

\section{Hyper-Parameters}

In order to be comparable with the state-of-the-art transformer based approach from the literature, we followed the same configuration as Baevski and Auli et al. [1]. We used 17 decoding layers, each layer with a 1024 hidden size, a 4096 feed-forward hidden size and 8 heads. The adaptive input factor was set to 4 . We set the $l_{\max }$ to 


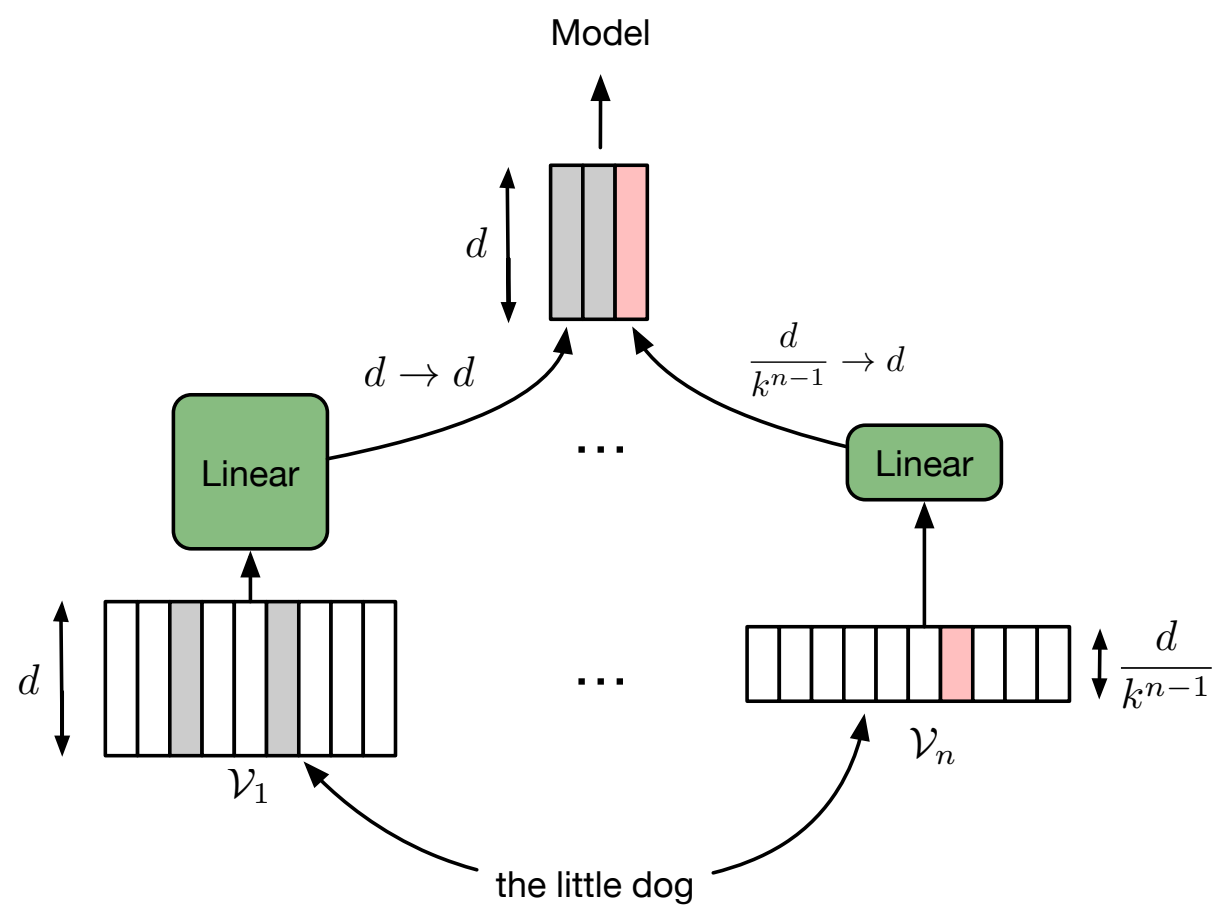

Figure 5.3: Words are assigned to clusters $\mathcal{V}_{i}$ based on their frequency which determines the size of the representations. Embeddings are projected to a common dimension $d$ before being fed to the model. Figure taken from [1]. 
$3,7,15,31 \times 4$ for each layer and the hyper-parameter $r_{\max }$ to zero.

\section{Optimization}

In order to train the language model, following Baevski and Auli et al. [1], we used Nesterov's accelerated gradient method proposed by Sutskever et al. [62] with a momentum of 0.99 and renormalizing gradients if their norm exceeds 0.1 (Pascanu et al. 63]). The learning rate is linearly warmed up from $10^{-7}$ to 1 for $16 \mathrm{~K}$ steps. Next, the learning rate is annealed using a cosine learning rate schedule with 4 cycles. Each cycle runs for twice the number of updates than the previous cycle and we lower the maximum and minimum learning rates by a rate 0.75 compared to the previous cycle. The initial minimum learning rate is $10^{-5}$ and the maximum is 1 . The model was trained for a total of $286 \mathrm{~K}$ steps.

\section{Hardware Details}

We trained the model using 8 NVIDIA RTX 2080 Ti GPUs using mixed-precision training. Each batch is using a maximum number of tokens equal to 4096. We used gradient accumulation to increase the batch size further by accumulating every two batches. This made the effective batch size to be of size $4096 * 8 * 2 \approx 65 \mathrm{~K}$ tokens.

\subsubsection{Results}

We evaluated our method on the task of language modeling. We considered the WikiText-103 benchmark dataset. We compared against recent methods in the literature. More specifically, we followed the setup that was implemented in the adaptive inputs baseline [1]. This work suggests the use of self-attention with adaptive input representations. We substituted the self-attention module with the proposed TaLK Convolution method. In order to assimilate the number of parameters used in their 
Table 5.6: Test perplexity on WikiText-103. We used adaptive inputs similar to [1 and show that our method yields better perplexity than self-attention using adaptive inputs.

\begin{tabular}{lcc}
\hline & Param & Test \\
\hline Neural Cache Model [64] & - & 40.8 \\
GCNN [45] & $229 \mathrm{M}$ & 37.2 \\
4 layer QRNN [65] & $151 \mathrm{M}$ & 33.0 \\
LSTM + Hebbian + Cache + MbPA 66] & - & 29.2 \\
Transformer + Adaptive Input [1] & $247 \mathrm{M}$ & 20.5 \\
\hline TaLK Convolution (Ours) & $240 \mathrm{M}$ & $\mathbf{2 0 . 3}$ \\
\hline
\end{tabular}

experiments, we increased the number of layers by one. As seen on Table 5.6, our method yields the best perplexity result. Moreover, we used a smaller number of parameters than the best comparison method. This is further evidence that our method can yield state-of-the-art results without the need of using self-attention.

\subsection{Abstractive Text Summarization}

The task of summarization is one of the most difficult tasks in NLP. The goal of summarization is to find elements of interest in a large corpus of text (e.g. documents) and produce a summary of the most important content. The two main types of summarization are the extractive and abstractive summarization. The goal of extractive summarization is to extract important sentences/words from the text and synthesize a summary based solely on text taken directly from the document by reording and concatenating the important extracted information. On the other hand, with abstractive summarization, the goal is to generate a completely new summary based on a model that comprehends the input document. These summaries may 
Table 5.7: CNN/DailyMail benchmark dataset for abstractive summarization.

\begin{tabular}{l|ccc}
\hline & Train & Validation & Test \\
\hline Examples & 287,226 & 13,368 & 11,490 \\
Vocabulary Size & & 30,000 & \\
\hline
\end{tabular}

using words that never seen in the documents.

Abstractive text summarization is more challenging than extractive and it is the task that we will focus on. For a model to be able to generate abstract summarizations it must also be able to comprehend a long text, often comprising of multiple sentences and/or paragraphs and to generate significantly shorter sentences that capture the essence of the article. This is a challenging task since the model needs to have a deep understanding of both the language and abstraction process over it.

\subsubsection{Datasets}

Despite the idea of abstractive summarization is old, only recently have people started working on this challenging task due to the revolution of deep learning. For this task, we decided to use the standard and widely used CNN/DailyMail benchmark dataset proposed by Hermann et al. [67]. The dataset was processed by Nallapati et al. [68 so it can be used for summarization. The dataset contains online news articles (781 tokens on average) paired with multi-sentence summaries (3.75 sentences or 56 tokens on average). Table 5.7 contains some statistics about the dataset. Specifically, about $287 \mathrm{~K}$ training examples are used to train the summarization model with $13 \mathrm{~K}$ examples specifically used for validation and $11 \mathrm{~K}$ for testing. We used Byte-PairEncoding to extract a sub-word vocabulary of a size of 30,000 tokens similar to $\mathrm{Wu}$ et al. [12]. Articles are truncated to 400 tokens (See et al. [69]). We evaluated using 
the F1-Rouge, more specifically the Rouge-1, Rouge-2 and Rouge-L metrics that were proposed by Lin $[70$. Following $\mathrm{Wu}$ et al. [12], we applied and appropriately tuned the maximum output length and we prohibited the repetition of the same trigram during generation. Finally, we applied a stepwise length penalty (Wu et al. [18]) which favors longer sentences.

\subsubsection{Experiment Details}

In this section, we describe the details of the experiments such the hyper-parameters the model were trained with, the optimization method as well the hardware details in order to ensure that our results are reproducible.

\section{Hyper-Parameters}

For this experiment, we trained two summarization models, namely Standard and Deep. Both models use 512, 1024 and 8 as the same hidden size, feed-forward hidden size and the number of heads respectively. The Standard configuration used 7 layers for the encoder and 6 layers for the decoder while the Deep model used 10 layers for both the encoder and the decoder. We set the $l_{\max }$ and $r_{\max }$ to $3,7,15,31 \times 4$ for each layer for the Standard model and $3,7,15,31 \times 7$ for the Deep model. For the decoder, we set the $l_{\max }$ to $3,7,15,31 \times 4$ for each layer for the Standard model and $3,7,15,31 \times 7$ for the Deep model and the hyper-parameter $r_{\max }$ to zero since we do not want the decoder to have access to future tokens.

\section{Optimization}

We used the Adam optimizer [51] with default values. In addition, our models were optimized using the cosine learning rate schedule [52] with a warmup of $10 \mathrm{~K}$ steps and a period of $20 \mathrm{~K}$ updates similar to the Machine Translation optimization strategy as 
described in Section 5.2.2. We set the maximum learning rate to 0.001. We applied dropout of 0.3 to the model and 0.1 to the TaLK Convolution relative offsets. Both models were trained for a total of $35 \mathrm{~K}$ steps.

\section{Hardware Details}

We trained all models using 8 NVIDIA RTX 2080 Ti GPUs using mixed-precision training. Each batch is using a maximum number of tokens equal to 3584 . We used gradient accumulation to increase the batch size further by accumulating every 16 batches. This made the effective batch size to be of size $3584 * 8 * 16 \approx 458 \mathrm{~K}$ tokens.

\subsubsection{Results}

We evaluated our proposed sequence modeling method on the task of abstractive summarization. We test the method's ability to process long documents on the CNN/DailyMail dataset. We encode an article of up to 400 sub-words and we generate a summarization composed from multiple sentences. Table 5.8 shows the results of our experiments. Our Standard model using the Rouge-1 and Rouge-2 metrics is able to outperform all previously proposed sequence modeling methods based on recurrent networks, convolution approaches and self-attention based models. In addition, the Standard model is using significantly less parameters, approximately 30M parameters less. The Deep model uses more layers to closely match the number of parameters of the baseline models. This deeper model is able to outperform all models in all metrics that it is evaluated with. This shows that our method is able to encode long sequences successfully without having the need to have access to all context as self-attention methods do. 


\begin{tabular}{lcrrr}
\hline Model & Param & Rouge-1 & Rouge-2 & Rouge-L \\
\hline LSTM [22 & - & 38.30 & 14.81 & 35.49 \\
CNN [71] & - & 39.06 & 15.38 & 35.77 \\
Self-attention Baseline [12] & $90 \mathrm{M}$ & 39.26 & 15.98 & 36.35 \\
Lightweight Convolution [12] & $86 \mathrm{M}$ & 39.52 & 15.97 & 36.51 \\
Dynamic Convolution [12 & $87 \mathrm{M}$ & 39.84 & 16.25 & 36.73 \\
\hline TaLK Convolution (Standard) & $59 \mathrm{M}$ & 40.03 & 18.45 & 36.13 \\
TaLK Convolution (Deep) & $83 \mathrm{M}$ & $\mathbf{4 0 . 5 9}$ & $\mathbf{1 8 . 9 7}$ & $\mathbf{3 6 . 8 1}$ \\
\hline
\end{tabular}

Table 5.8: Results on CNN/DailyMail summarization.

\subsection{Sentence Classification}

To further evaluate the proposed method, we decided to conduct an experiment comparing how different state-of-the-art methods perform in the task of classifying a sentence. Specifically, we chose to classify sentences based on the binary sentiment they correspond to. Sentiment classification is considered a classic NLP task and it is a very well studied problem.

\subsubsection{Datasets}

Perhaps the most famous sentiment classification dataset is the IMDB Movies Reviews benchmark dataset. The dataset consists of 50,000 movie reviews which are categorized as being either positive or negative. We use 25,000 reviews for training and the rest for testing (Maas et al. [72]). We used byte-pair-encoding to extract a vocabulary of size 50,260 sub-word tokens. 


\subsubsection{Experiment Details}

In this section, we describe the details of the experiment including the hyperparameters the model was trained with, the optimization method as well the hardware details in order to ensure that our results are reproducible.

\section{Hyper-Parameters}

We trained four models, a Transformer model [13], a Lightweight Convolution model [12], a Dynamic Convolution model [12] and our proposed method TaLK Convolution. For all four models, we used 7 encoding layers, each with a 512 hidden size, a 512 feed-forward hidden size and 4 heads. We set the $l_{\max }$ and $r_{\max }$ to $3,7,15,31 \times 4$ for each layer. The output of the encoder network is averaged across the time dimension and the final representation is passed to two connected layers of size 512 and a ReLU activation function in between.

\section{Optimization}

We used the Adam optimizer with default values. Additionally, we optimized the models using the polynomial learning rate decay. The maximum learning rate was set to 0.00001 . The models were trained for a total of 10 epochs.

\section{Hardware Details}

We trained all models using a single NVIDIA RTX 2080 Ti GPU using mixed-precision training. Each batch uses a maximum number of tokens equal to 4400. We used gradient accumulation to increase the batch size further by accumulating every two batches. This made the effective batch size to be of size $4400 * 1 * 2 \approx 8800$ tokens. 


\begin{tabular}{lcrrr}
\hline Model & Param & Accuracy & Sent/sec & Tok/sec \\
\hline Self-attention Baseline & $38 \mathrm{M}$ & $86.96 \%$ & 51.8 & 29596.7 \\
Lightweight Convolution & $34 \mathrm{M}$ & $86.87 \%$ & 90.8 & 42353.1 \\
Dynamic Convolution & $35 \mathrm{M}$ & $87.34 \%$ & 78.1 & 35135.2 \\
\hline TaLK Convolution & $34 \mathrm{M}$ & $\mathbf{8 7 . 9 1 \%}$ & $\mathbf{9 1 . 2}$ & $\mathbf{4 2 5 1 8 . 4}$ \\
\hline
\end{tabular}

Table 5.9: Results on IMDB Movies Reviews dataset.

\subsubsection{Results}

In this section, we present the results of the sentiment classification task. Table 5.9 shows the accuracy our method achieves compared to other state-of-the-art nonautoregressive methods in the literature. Our method is able to achieve better accuracy with the least number of parameters. In addition, we report the sentences per second and the tokens per second our method is able to process during inference. These metrics show that our method is, in fact, faster than the other self-attention and convolution based methods from the literature.

\subsection{Ablation Study}

In order to evaluate the importance of the different choices for the TaLK Convolutions, we varied our baseline model, described in Section 4.4, using the different proposed extensions mentioned in Sections 4.5 and 4.6. We measured the performance on the validation set of the IWSLT De-En translation benchmark dataset. We used beam search as described in Section 5.2.1. We report the results in Table 5.10 .

Initially, we modified the baseline model with the addition of the output normalization (Section 4.5). As seen in Table 5.10, the original method is not able to 
Table 5.10: Ablation on IWSLT De-En validation set. $(+)$ indicates that a result includes all preceding features.

\begin{tabular}{lrr}
\hline Model & Param & BLEU \\
\hline TaLK Convolution $\left(a_{i}^{l}, a_{i}^{r}=1 \mathrm{x} 7, H=1\right)$ & $42 \mathrm{M}$ & diverges \\
+ Output Normalization & $42 \mathrm{M}$ & $35.70 \pm 0.1$ \\
+ Increasing Max Offsets $\left(a_{i}^{l}, a_{i}^{r}=1,3,7,15 \mathrm{x} 4\right)$ & $42 \mathrm{M}$ & $36.23 \pm 0.1$ \\
+ Offsets Dropout $(p=0.1)$ & $42 \mathrm{M}$ & $36.37 \pm 0.05$ \\
+ Fully-headed Kernels $(H=512)$ & $47 \mathrm{M}$ & $36.51 \pm 0.07$ \\
+ Multi-headed Kernels $(H=4)$ & $42 \mathrm{M}$ & $36.65 \pm 0.05$ \\
\hline
\end{tabular}

converge. This validates our intuition that since we are summing the available information inside the kernel, not normalized outputs make learning difficult for the layers that follow. Next, we increased the values $l_{\max }, r_{\max }$ to allow larger adaptive kernel sizes which yielded a higher performance without additional computation cost. Further, we introduced a dropout unit with probability $p=0.1$ on the generated relative offsets. This allowed for the performance to increase further as we stopped the model from overfitting over the same window size. Next, we increased the number of heads $H$ from 1 to 512 (all available dimensions) and we called this fully-head TaLK Convolution. We can see that by treating each of the 512 dimensions separately and generating 512 relative offsets, we were able to increase the performance. However, we believe that by having each dimension generate its own offsets actually brings some noise. Thus, we reduced the number of heads to $H=4$ which increased the performance even more. 
Table 5.11: Throughput and memory consumption decrease measured for different sequence lengths $(n)$ on a batch of size 10 with each token being represented with $d=1024$ and $H=16$. Throughput is calculated across $100 \mathrm{~K}$ iterations of a single input encoding execution for each method. Memory decrease is computed as how many times less memory we need to encoding the input embedding compared to Self-Attention. Larger numbers indicate better performance.

\begin{tabular}{l|cc|cc|cc|cc}
\hline \multirow{2}{*}{ Method } & \multicolumn{2}{|c|}{$n=10$} & \multicolumn{2}{c|}{$n=100$} & \multicolumn{2}{c}{$n=1,000$} & \multicolumn{2}{c}{$n=10,000$} \\
\cline { 2 - 9 } & iter/sec & Mem. $\downarrow$ & iter/sec & Mem. $\downarrow$ & iter/sec & Mem. $\downarrow$ & iter/sec & Mem. $\downarrow$ \\
\hline Self-Attention & 4576 & $1 \mathrm{x}$ & 3437 & $1 \mathrm{x}$ & 102 & $1 \mathrm{x}$ & OOM & $1 \mathrm{x}$ \\
DynamicConv $(k=3)$ & 3739 & $1 \mathrm{x}$ & 3308 & $0.99 \mathrm{x}$ & 443 & $2.8 \mathrm{x}$ & 45 & $25.4 \mathrm{x}$ \\
DynamicConv $(k=31)$ & 4535 & $0.97 \mathrm{x}$ & 3860 & $1 \mathrm{x}$ & 325 & $2.7 \mathrm{x}$ & 29 & $20.2 \mathrm{x}$ \\
\hline TaLK Convolution & $\mathbf{9 6 8 6}$ & $\mathbf{1 . 1 x}$ & $\mathbf{6 1 2 6}$ & $\mathbf{1 . 1 x}$ & $\mathbf{8 9 8}$ & $\mathbf{3 . 1 x}$ & $\mathbf{9 2}$ & $\mathbf{2 6 . 4 x}$ \\
\hline
\end{tabular}

\subsection{Encoding Inference Speed Comparison}

We also compared our method against other non-autoregressive methods in terms of encoding inference speed and memory consumption. We measured the speed using a single NVIDIA RTX 2080 Ti GPU with full precision floating-point arithmetic (FP32). Specifically, we measured the throughput of encoding a batch of size $B=10$, $d=1024$ and $H=16$. For each method, we only took into consideration the time it takes to process using the core approach of each encoding method.

For self-attention [13], we only timed the attention operation $\operatorname{softmax}\left(\frac{Q K^{T}}{\sqrt{d_{k}}}\right) V$. For dynamic convolutions 12 , we only timed the operation $\operatorname{Depthwise} \operatorname{Conv}\left(X, \operatorname{softmax}\left(W_{\mathrm{dyn}}\right), i, c\right)$ where $W_{\mathrm{dyn}} \in \mathbb{R}^{n \cdot B \cdot H \times K}$ is the generated kernel for each time-step. The authors of dynamic convolutions proposed two ways of implementing their method. The first method uses the standard convolution unfolding function which is faster for longer sequences. The second approach is the 
band matrix trick method which copies and expands the normalized weights matrix into a band matrix. This second approach yields faster execution time for shorter sequences but is more memory intensive. In order to be fair, in our experiments we used unfolding to sequences longer than 500 tokens and band matrices for shorter sequences. We also set $K$ to 3 and 31 , the first being the smallest kernel size dynamic convolutions use and the second being the largest. Finally, for our method we measured the time to compute the large kernel convolution operation given the relative offsets. We evaluated for $100 \mathrm{~K}$ iterations across four different sequence lengths $n$.

Table 5.11 shows that our method yields much better throughput than all other methods. Specifically, the number of iterations of self-attention per second is comparable to dynamic convolutions for short sentences $(n<500)$. Our method allows for more sentences to be processed each second, leading to a much higher throughput. For longer sentences, self-attention is notably slower than our method and for the case of $n=10,000$, self-attention was running out-of-memory and was not able to execute an iteration. Although our method has a logarithmic time for computing the summed-area table (Section 4.9), due to the fact that we are computing a much "cheaper" operation in terms of complexity (as we only utilize additions) whereas other methods employ multiplication as well as addition operations. Therefore, our method has a considerably higher throughput compared to dynamic convolutions.

Furthermore, we examined the running memory requirements for all three different non-autoregressive methods. We compared dynamic convolutions and our proposed method against self-attention and report the number of times we reduced the running memory compared to self-attention. For all sequence length cases, our method requires less memory than dynamic convolutions when compared to the "expensive" self-attention operation. The times we were able to decrease the memory consumption 
can be seen on Table 5.11 .

\subsection{Conclusion}

In this chapter, we described our experimental evaluation of our proposed sequence modeling approach. We evaluated based on four tasks: machine translation, language modeling, abstractive summarization and sentence classification. Moreover, we conducted an ablation study and we showed that our proposed extensions to the base TaLK Convolution operation are indeed help the model to yield better results. Finally, we analyzed the encoding inference speed and exhibited the faster execution time that our method can provide even for very long sequences. 


\section{Chapter 6}

\section{Conclusion}

\subsection{Summary and Conclusion}

In this thesis, our aim was to explore new methods of processing sequences in linear time. We discussed how attention is considered the standard approach that all state-of-the-art methods employ and that this technique, although it yields good accuracy, is expensive both in terms of memory as well as running time. Additionally, we went over recent approaches that suggest a type of convolution that has a learnable kernel size.

To overcome the quadratic time complexity self-attention methods have, we proposed a novel sequence modeling method, namely Time-aware Large Kernel (TaLK) Convolution, which can process a sequence in linear time and is not based on attention. This novel convolution operation is based on learning to generate a kernel size conditioned on the input sequence elements. We took advantage of the summed-area table operation to expedite the convolution process and implemented our own low-level CUDA primitives in $\mathrm{C}++$ to be able to support our method with Pytorch, a popular deep learning framework. Furthermore, we proposed extensions to the base idea to make it stable and improve the results. We showed how our 
method can be used for decoding and compared the time complexity against all previously proposed methods in the literature.

In order to demonstrate the effectiveness of TaLK Convolutions, we evaluated our method in four fundamental natural language processing tasks. Specifically, we tested the proposed approach against three benchmark datasets in machine translation and showed that our method can achieve state-of-the-art results. Additionally, we tested our method in language modeling and abstractive summarization where we were able to set a new best score in both tasks. Moreover, we verified that TaLK Convolutions can yield state-of-the-art results in sentence classification with faster execution time. We ran an ablation study and showed that our proposed extensions are indeed useful. Finally, we demonstrated that our method is capable of modeling more sequences per second with a smaller memory footprint even for long sequences.

\subsection{Future Work}

To our knowledge, we are the first to propose and apply in the area of natural language processing, a convolution operation with an adaptive kernel size. Several potential directions can be explored to further improve the proposed method.

More research should explore different ways of applying the TaLK Convolution operation in a non-contiguous way. This would help to connect elements from the sequences that are not in the same kernel window. Furthermore, a more optimized version of the implementation could be examined where the summed-area table operation and the operation of applying the offsets are merged. Finally, we would like to apply our sequence modeling method to other tasks such as video processing and time-series data. 


\section{List of References}

[1] A. Baevski and M. Auli, "Adaptive input representations for neural language modeling," in International Conference on Learning Representations, 2019.

[2] L. Dormehl, "What is an artificial neural network? here's everything you need to know," https://www.digitaltrends.com/cool-tech/what-is-an-artificial-neuralnetwork, 2019.

[3] Y. Qiu, Y. Liu, J. S. Arteaga-Falconi, H. Dong, and A. E. Saddik, "Evm-cnn: Real-time contactless heart rate estimation from facial video," IEEE Transactions on Multimedia, vol. 21, pp. 1778-1787, 2019.

[4] S.-H. Tsang, "Review: Xception - with depthwise separable convolution, better than inception-v3 (image classification)," https://towardsdatascience.com/review-xception-with-depthwise-separableconvolution-better-than-inception-v3-image-dc967dd42568, 2018.

[5] F. Yu and V. Koltun, "Multi-scale context aggregation by dilated convolutions," 2015.

[6] C. Olah, "Understanding lstm networks," http://colah.github.io/posts/2015-08Understanding-LSTMs/, 2015.

[7] A. A. Ismail, T. Wood, and H. C. Bravo, "Improving long-horizon forecasts with expectation-biased lstm networks," 2018.

[8] I. Sutskever, O. Vinyals, and Q. V. Le, "Sequence to sequence learning with neural networks," in Proceedings of the 27th International Conference on Neural Information Processing Systems - Volume 2, NIPS'14, (Cambridge, MA, USA), p. 3104-3112, MIT Press, 2014.

[9] K. He, X. Zhang, S. Ren, and J. Sun, "Deep residual learning for image recognition," 2015. 
[10] Łukasz Kaiser and I. Sutskever, "Neural gpus learn algorithms," 2015.

[11] J. Gehring, M. Auli, D. Grangier, D. Yarats, and Y. N. Dauphin, "Convolutional sequence to sequence learning," 2017.

[12] F. Wu, A. Fan, A. Baevski, Y. N. Dauphin, and M. Auli, "Pay less attention with lightweight and dynamic convolutions," 2019.

[13] A. Vaswani, N. Shazeer, N. Parmar, J. Uszkoreit, L. Jones, A. N. Gomez, L. Kaiser, and I. Polosukhin, "Attention is all you need," 2017.

[14] N. Kitaev, L. Kaiser, and A. Levskaya, "Reformer: The efficient transformer," in International Conference on Learning Representations, 2020.

[15] E. Burkov and V. Lempitsky, "Deep neural networks with box convolutions," in Advances in Neural Information Processing Systems 31, 2018.

[16] L. Zhang, M. Halber, and S. Rusinkiewicz, "Accelerating large-kernel convolution using summed-area tables," 2019.

[17] D. Bahdanau, K. Cho, and Y. Bengio, "Neural machine translation by jointly learning to align and translate," 2014.

[18] Y. Wu, M. Schuster, Z. Chen, Q. V. Le, M. Norouzi, W. Macherey, M. Krikun, Y. Cao, Q. Gao, K. Macherey, J. Klingner, A. Shah, M. Johnson, X. Liu, Łukasz Kaiser, S. Gouws, Y. Kato, T. Kudo, H. Kazawa, K. Stevens, G. Kurian, N. Patil, W. Wang, C. Young, J. Smith, J. Riesa, A. Rudnick, O. Vinyals, G. Corrado, M. Hughes, and J. Dean, "Google's neural machine translation system: Bridging the gap between human and machine translation," 2016.

[19] Y. Kim, "Convolutional neural networks for sentence classification," in Proceedings of the 2014 Conference on Empirical Methods in Natural Language Processing (EMNLP), Association for Computational Linguistics, 2014.

[20] N. Kalchbrenner, E. Grefenstette, and P. Blunsom, "A convolutional neural network for modelling sentences," in Proceedings of the 52nd Annual Meeting of the Association for Computational Linguistics (Volume 1: Long Papers), Association for Computational Linguistics, 2014.

[21] N. Kalchbrenner, L. Espeholt, K. Simonyan, A. van den Oord, A. Graves, and K. Kavukcuoglu, "Neural machine translation in linear time," 2016. 
[22] R. Paulus, C. Xiong, and R. Socher, "A deep reinforced model for abstractive summarization," 2017.

[23] Z. Dai, Z. Yang, Y. Yang, J. Carbonell, Q. Le, and R. Salakhutdinov, "Transformer-xl: Attentive language models beyond a fixed-length context," Proceedings of the 57th Annual Meeting of the Association for Computational Linguistics, 2019.

[24] I. Goodfellow, Y. Bengio, and A. Courville, Deep Learning. MIT Press, 2016.

[25] R. Sennrich, B. Haddow, and A. Birch, "Neural machine translation of rare words with subword units," in Proceedings of the 54th Annual Meeting of the Association for Computational Linguistics (Volume 1: Long Papers), (Berlin, Germany), pp. 1715-1725, Association for Computational Linguistics, Aug. 2016.

[26] K. Cho, B. van Merriënboer, C. Gulcehre, D. Bahdanau, F. Bougares, H. Schwenk, and Y. Bengio, "Learning phrase representations using RNN encoder-decoder for statistical machine translation," in Proceedings of the 2014 Conference on Empirical Methods in Natural Language Processing (EMNLP), (Doha, Qatar), pp. 1724-1734, Association for Computational Linguistics, Oct. 2014.

[27] H. Scheidl, "Beam search decoding in ctc-trained neural networks," https://towardsdatascience.com/beam-search-decoding-in-ctc-trained-neuralnetworks-5a889a3d85a \%, 2018.

[28] T. Luong, H. Pham, and C. D. Manning, "Effective approaches to attentionbased neural machine translation," Proceedings of the 2015 Conference on Empirical Methods in Natural Language Processing, 2015.

[29] B. Zhang, D. Xiong, and J. Su, "A gru-gated attention model for neural machine translation," 2017.

[30] G. Zhong, G. Yue, and X. Ling, "Recurrent attention unit," 2018.

[31] R. Dey and F. M. Salemt, "Gate-variants of gated recurrent unit (gru) neural networks," in 2017 IEEE 60th International Midwest Symposium on Circuits and Systems (MWSCAS), pp. 1597-1600, Aug 2017.

[32] X. Zhang, J. Su, Y. Qin, Y. Liu, R. Ji, and H. Wang, "Asynchronous bidirectional decoding for neural machine translation," 2018. 
[33] J. Zhou, Y. Cao, X. Wang, P. Li, and W. Xu, "Deep recurrent models with fast-forward connections for neural machine translation," 2016.

[34] Y. N. Dauphin, A. Fan, M. Auli, and D. Grangier, "Language modeling with gated convolutional networks," 2016.

[35] K. He, X. Zhang, S. Ren, and J. Sun, "Deep residual learning for image recognition," 2016 IEEE Conference on Computer Vision and Pattern Recognition (CVPR), 2016.

[36] C. Szegedy, S. Ioffe, V. Vanhoucke, and A. Alemi, "Inception-v4, inception-resnet and the impact of residual connections on learning," in AAAI, 2016.

[37] Y. Lecun, L. Bottou, Y. Bengio, and P. Haffner, "Gradient-based learning applied to document recognition," Proceedings of the IEEE, 1998.

[38] J. T. Springenberg, A. Dosovitskiy, T. Brox, and M. Riedmiller, "Striving for simplicity: The all convolutional net," International Conference on Learning Representations, 2015.

[39] F. C. Crow, "Summed-area tables for texture mapping," in Proceedings of the 11th Annual Conference on Computer Graphics and Interactive Techniques, 1984.

[40] P. Viola and M. Jones, "Robust real-time object detection," in International Journal of Computer Vision, 2001.

[41] R. E. Ladner and M. J. Fischer, "Parallel prefix computation," J. ACM, 1980.

[42] U. Vishkin, "Prefix sums and an application thereof," vol. : 09/224,104, 2003/04/01/ 2003.

[43] G. E. Hinton, N. Srivastava, A. Krizhevsky, I. Sutskever, and R. R. Salakhutdinov, "Improving neural networks by preventing co-adaptation of feature detectors," 2012.

[44] N. Srivastava, G. Hinton, A. Krizhevsky, I. Sutskever, and R. Salakhutdinov, "Dropout: A simple way to prevent neural networks from overfitting," Journal of Machine Learning Research, 2014.

[45] Y. N. Dauphin, A. Fan, M. Auli, and D. Grangier, "Language modeling with gated convolutional networks," in Proceedings of the 34 th International Conference on Machine Learning - Volume 70, 2017. 
[46] P. Ramachandran, B. Zoph, and Q. V. Le, "Searching for activation functions," 2017.

[47] J. F. Kolen and S. C. Kremer, Gradient Flow in Recurrent Nets: The Difficulty of Learning LongTerm Dependencies. IEEE, 2001.

[48] G. Tang, M. Müller, A. Rios, and R. Sennrich, "Why self-attention? a targeted evaluation of neural machine translation architectures," Proceedings of the 2018 Conference on Empirical Methods in Natural Language Processing, 2018.

[49] S. Edunov, M. Ott, M. Auli, D. Grangier, and M. Ranzato, "Classical structured prediction losses for sequence to sequence learning," in Proceedings of the 2018 Conference of the North American Chapter of the Association for Computational Linguistics: Human Language Technologies, Volume 1 (Long Papers), (New Orleans, Louisiana), pp. 355-364, Association for Computational Linguistics, June 2018.

[50] K. Papineni, S. Roukos, T. Ward, and W.-J. Zhu, "Bleu: a method for automatic evaluation of machine translation," in Proceedings of the 40th annual meeting on association for computational linguistics, pp. 311-318, Association for Computational Linguistics, 2002.

[51] D. P. Kingma and J. Ba, "Adam: A method for stochastic optimization," in International Conference on Learning Representations, 2015.

[52] I. Loshchilov and F. Hutter, "Sgdr: Stochastic gradient descent with warm restarts," in International Conference on Learning Representations, 2017.

[53] M. Popel and O. Bojar, "Training tips for the transformer model," The Prague Bulletin of Mathematical Linguistics, vol. 110, p. 43-70, Apr 2018.

[54] P. Micikevicius, S. Narang, J. Alben, G. Diamos, E. Elsen, D. Garcia, B. Ginsburg, M. Houston, O. Kuchaiev, G. Venkatesh, and H. Wu, "Mixed precision training," International Conference on Learning Representations, 2018.

[55] K. Ahmed, N. S. Keskar, and R. Socher, "Weighted transformer network for machine translation," 2017.

[56] M. X. Chen, O. Firat, A. Bapna, M. Johnson, W. Macherey, G. Foster, L. Jones, M. Schuster, N. Shazeer, N. Parmar, A. Vaswani, J. Uszkoreit, L. Kaiser, Z. Chen, $\mathrm{Y}$. Wu, and M. Hughes, "The best of both worlds: Combining recent advances in neural machine translation," in Proceedings of the 56th Annual Meeting of the Association for Computational Linguistics (Volume 1: Long Papers), 2018. 
[57] P. Shaw, J. Uszkoreit, and A. Vaswani, "Self-attention with relative position representations," Proceedings of the 2018 Conference of the North American Chapter of the Association for Computational Linguistics: Human Language Technologies, Volume 2 (Short Papers), 2018.

[58] M. Ott, S. Edunov, D. Grangier, and M. Auli, "Scaling neural machine translation," Proceedings of the Third Conference on Machine Translation: Research Papers, 2018.

[59] Y. Deng, Y. Kim, J. Chiu, D. Guo, and A. M. Rush, "Latent alignment and variational attention," in Proceedings of the 32nd International Conference on Neural Information Processing Systems, 2018.

[60] J. Devlin, M.-W. Chang, K. Lee, and K. Toutanova, "Bert: Pre-training of deep bidirectional transformers for language understanding," 2018.

[61] S. Merity, C. Xiong, J. Bradbury, and R. Socher, "Pointer sentinel mixture models," 2016.

[62] I. Sutskever, J. Martens, G. Dahl, and G. Hinton, "On the importance of initialization and momentum in deep learning," in Proceedings of the 30th International Conference on Machine Learning (S. Dasgupta and D. McAllester, eds.), vol. 28 of Proceedings of Machine Learning Research, (Atlanta, Georgia, USA), pp. 1139-1147, PMLR, 17-19 Jun 2013.

[63] R. Pascanu, T. Mikolov, and Y. Bengio, "On the difficulty of training recurrent neural networks," in Proceedings of the 30th International Conference on Machine Learning (S. Dasgupta and D. McAllester, eds.), vol. 28 of Proceedings of Machine Learning Research, (Atlanta, Georgia, USA), pp. 1310-1318, PMLR, 17-19 Jun 2013.

[64] E. Grave, A. Joulin, and N. Usunier, "Improving neural language models with a continuous cache," in International Conference on Learning Representations, 2017.

[65] S. Merity, N. S. Keskar, and R. Socher, "An analysis of neural language modeling at multiple scales," 2018.

[66] J. W. Rae, C. Dyer, P. Dayan, and T. P. Lillicrap, "Fast parametric learning with activation memorization," in $I C M L, 2018$.

[67] K. M. Hermann, T. Kočiský, E. Grefenstette, L. Espeholt, W. Kay, M. Suleyman, and P. Blunsom, "Teaching machines to read and comprehend," 2015. 
[68] R. Nallapati, B. Zhou, C. dos Santos, Ç. Guİłlçehre, and B. Xiang, "Abstractive text summarization using sequence-to-sequence RNNs and beyond," in Proceedings of The 20th SIGNLL Conference on Computational Natural Language Learning, (Berlin, Germany), pp. 280-290, Association for Computational Linguistics, Aug. 2016.

[69] A. See, P. J. Liu, and C. D. Manning, "Get to the point: Summarization with pointer-generator networks," Proceedings of the 55th Annual Meeting of the Association for Computational Linguistics (Volume 1: Long Papers), 2017.

[70] C.-Y. Lin, "ROUGE: A package for automatic evaluation of summaries," in Text Summarization Branches Out, (Barcelona, Spain), pp. 74-81, Association for Computational Linguistics, July 2004.

[71] A. Fan, D. Grangier, and M. Auli, "Controllable abstractive summarization," in Proceedings of the 2nd Workshop on Neural Machine Translation and Generation, (Melbourne, Australia), pp. 45-54, Association for Computational Linguistics, July 2018.

[72] A. L. Maas, R. E. Daly, P. T. Pham, D. Huang, A. Y. Ng, and C. Potts, "Learning word vectors for sentiment analysis," in Proceedings of the 49th Annual Meeting of the Association for Computational Linguistics: Human Language Technologies, (Portland, Oregon, USA), pp. 142-150, Association for Computational Linguistics, June 2011. 


\section{Appendix A}

\section{TaLK Convolution CUDA Pseudocode}

In this section, we describe the implemented low-level CUDA primitives that were developed in order to be able to train the proposed Time-aware Large Kernel Convolutions.

\section{A.1 Encoder-optimized Implementation}

\section{A.1.1 Forward Implementation}

The pseudocode below describes the CUDA kernel implemented in $\mathrm{C}++$ to compute the forward propagation of the proposed TaLK Convolution operation. It takes as input the input sequence tensor, the left and right offset tensors, the $l_{\max }$ and $r_{\max }$ values and the output tensor to store the results. This implementation is used for the encoder model.

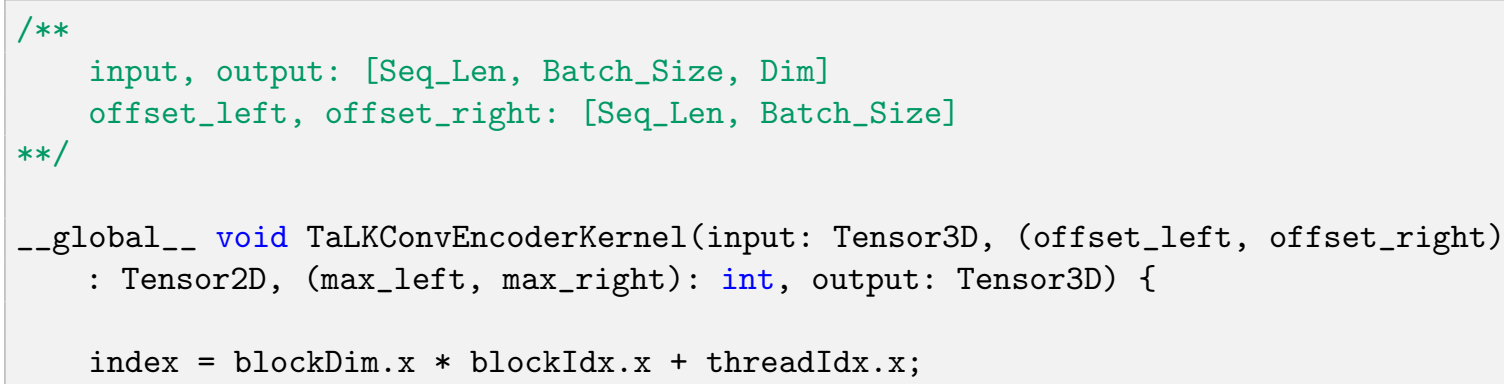




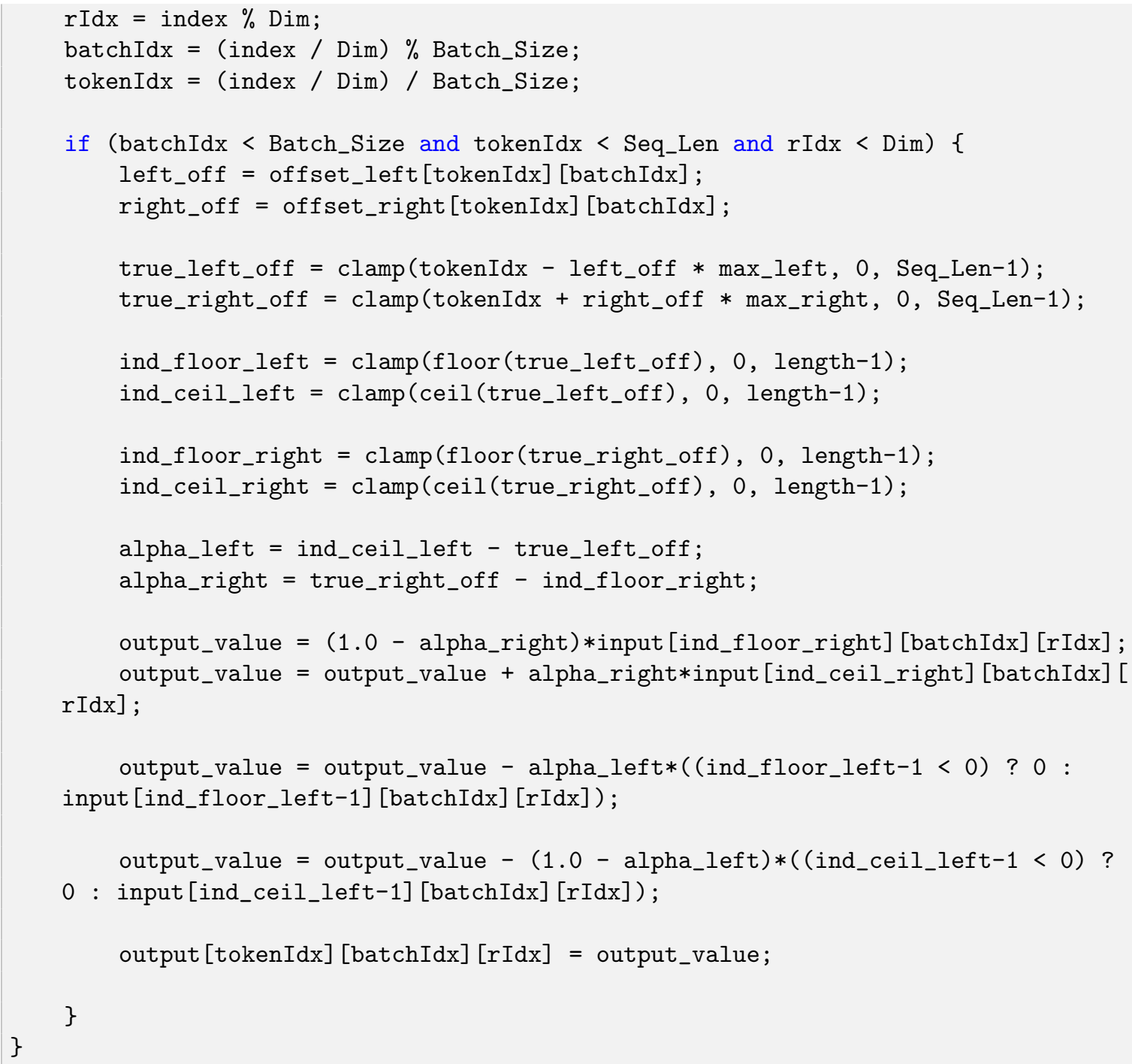

\section{A.1.2 Backward Implementation}

Below is the implementation of the backwards direction of the encoder-optimized implementation of TaLK Convolutions. This function is responsible to compute the gradients with respect of the input tensor and the two offset tensors given the gradient calculated up this stage. 


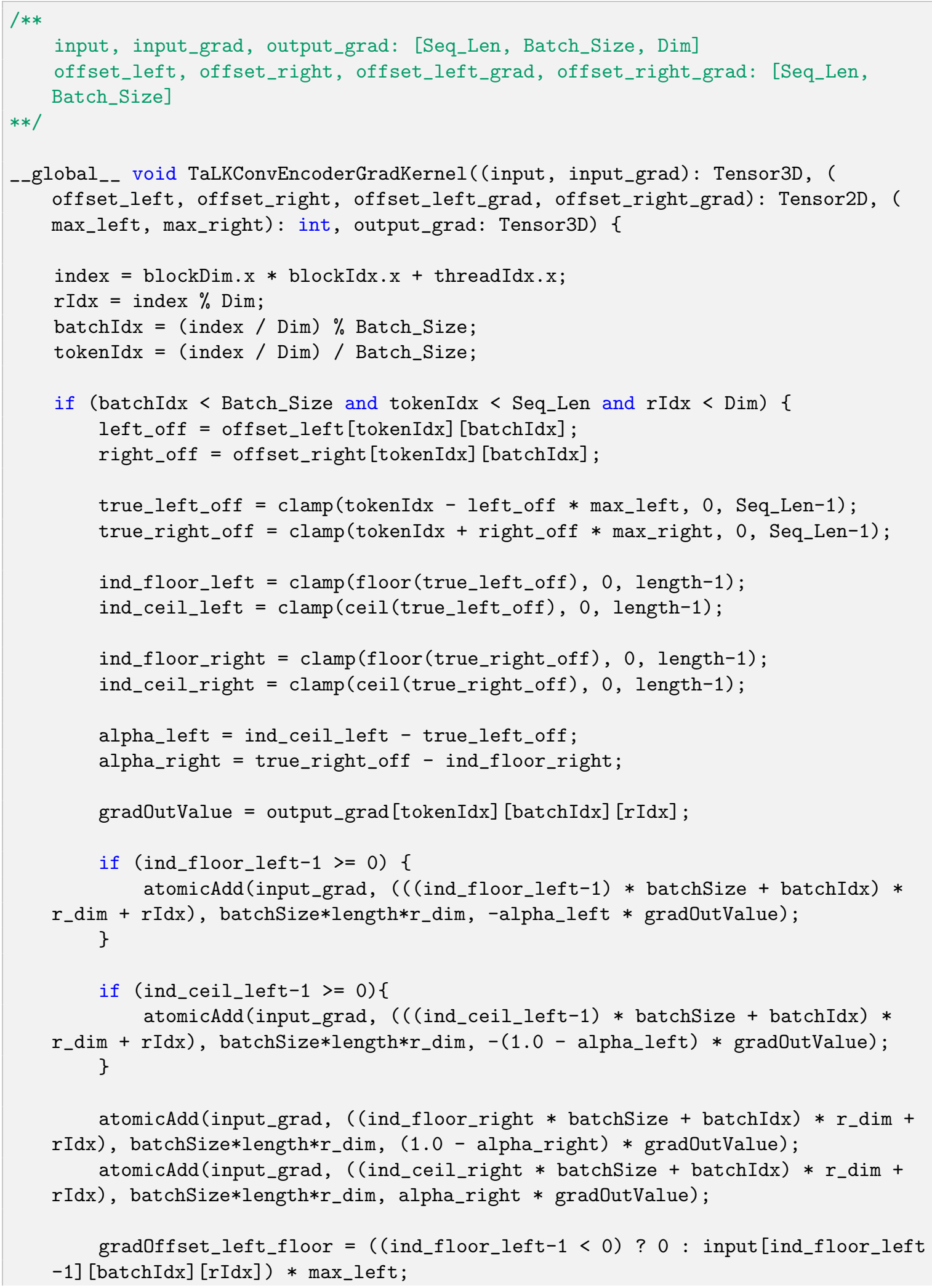




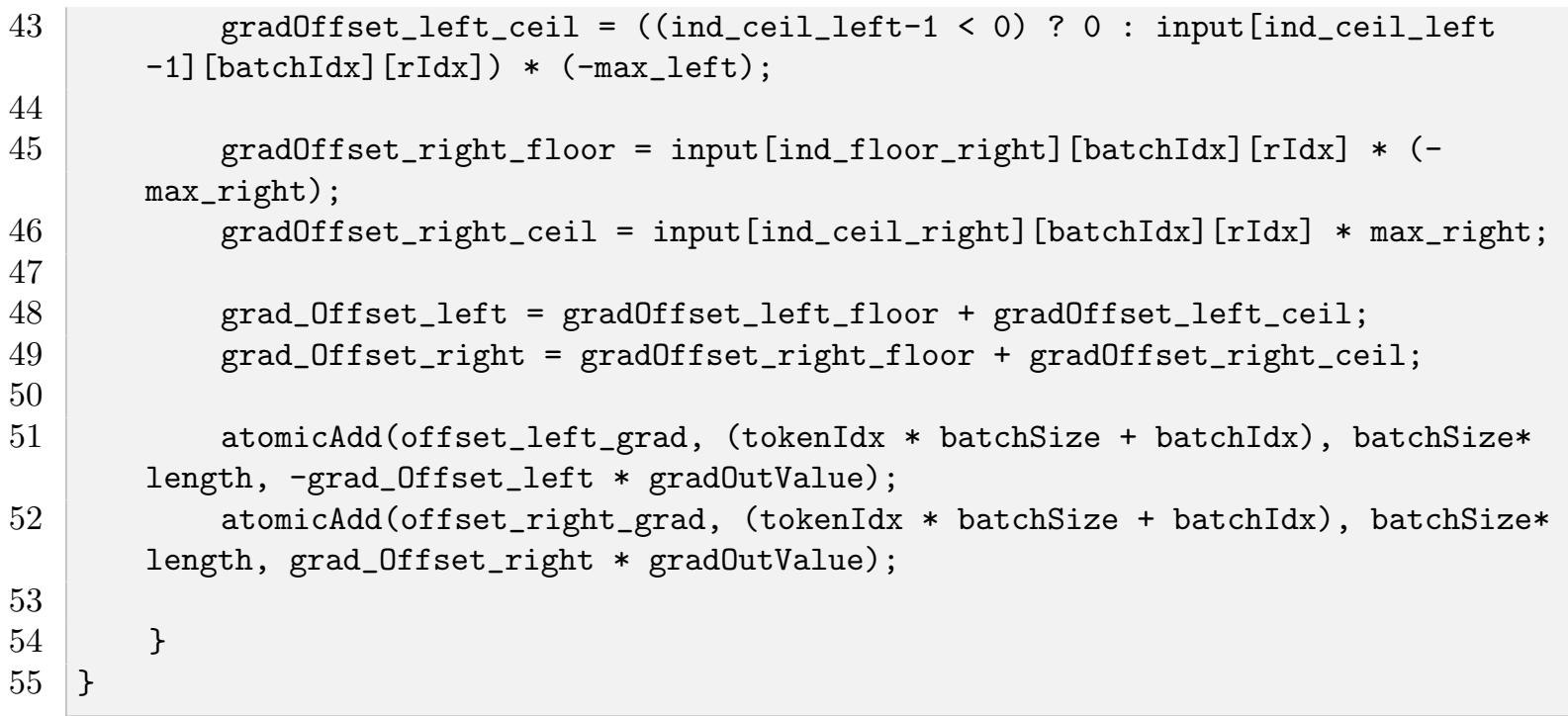

\section{A.2 Decoder-optimized Implementation}

\section{A.2.1 Forward Implementation}

Since during decoding, we are only interested in combining past timesteps, we can optimize the computation further by only using the left offsets and assuming that the right offsets are zero. Below, we present a modified version of the forward propagation CUDA kernel that implements this optimization.

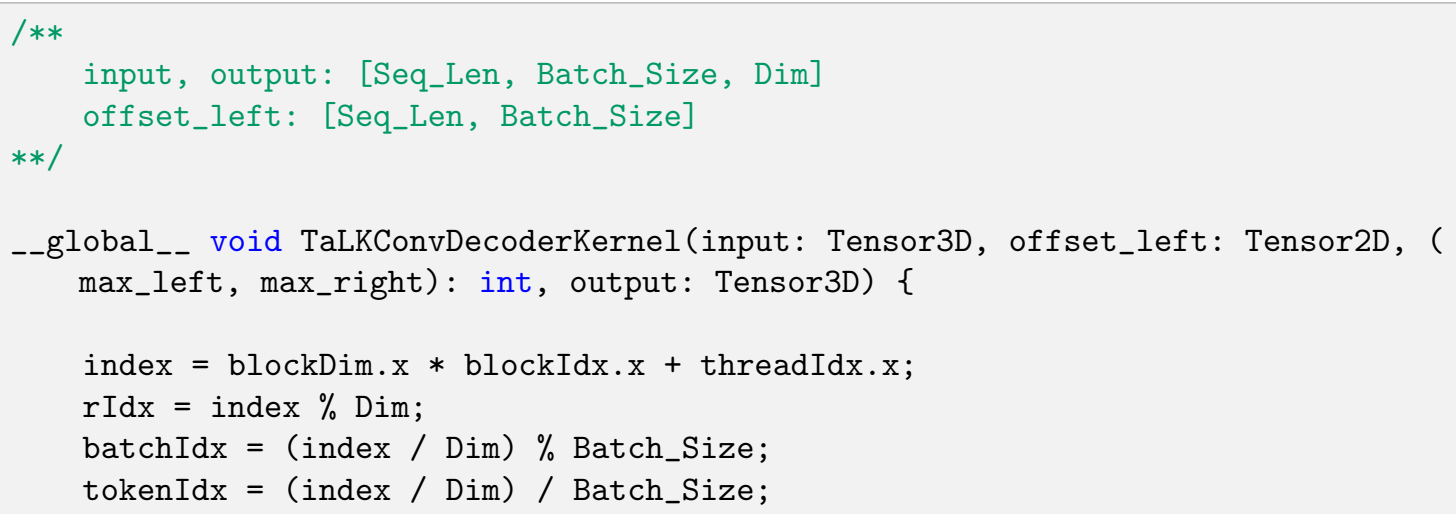




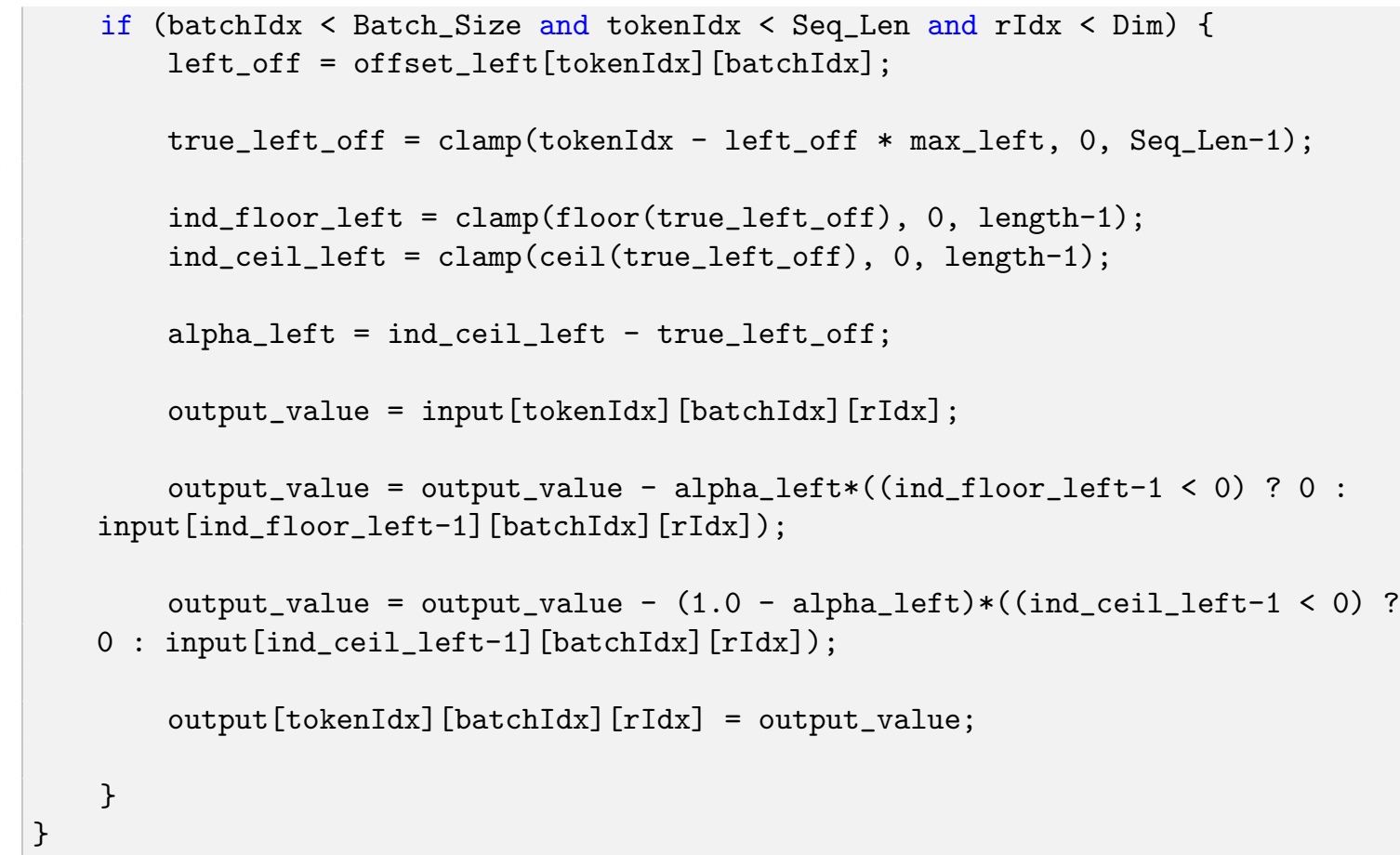

\section{A.2.2 Backward Implementation}

Below, we describe the CUDA kernel pseudocode for calculating the gradients using the optimized version for decoding faster.

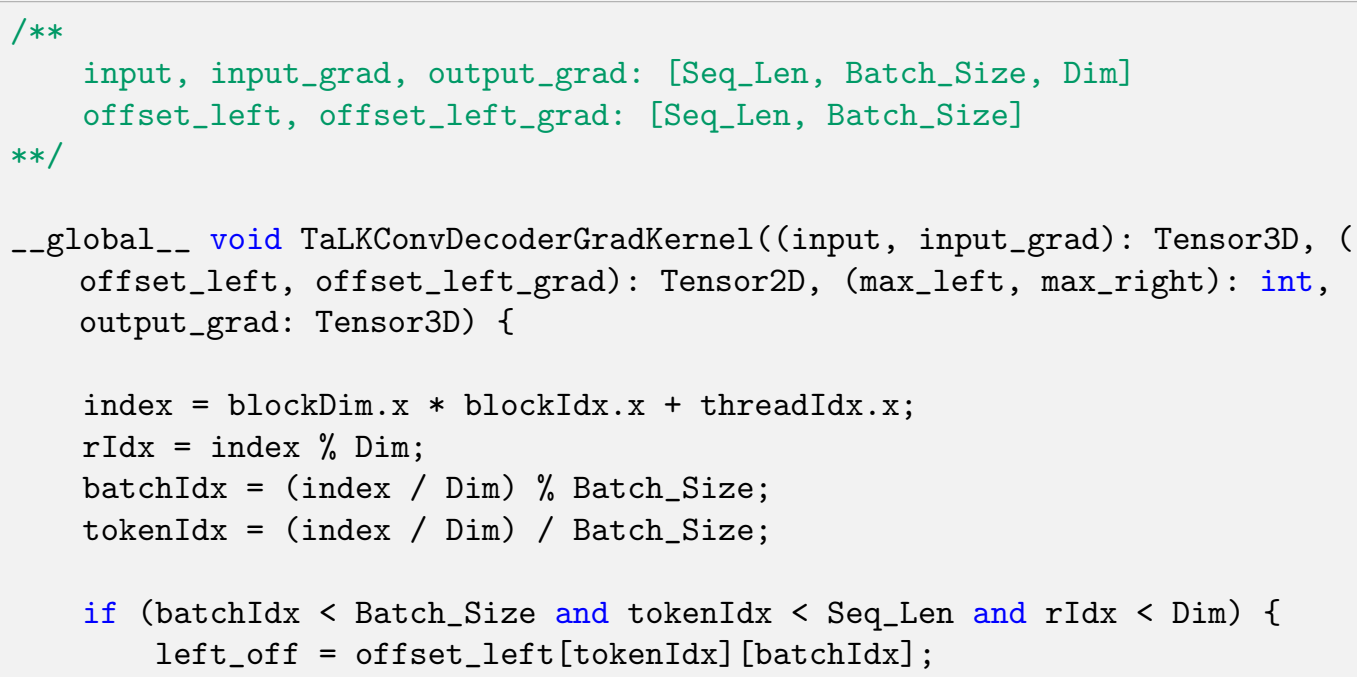




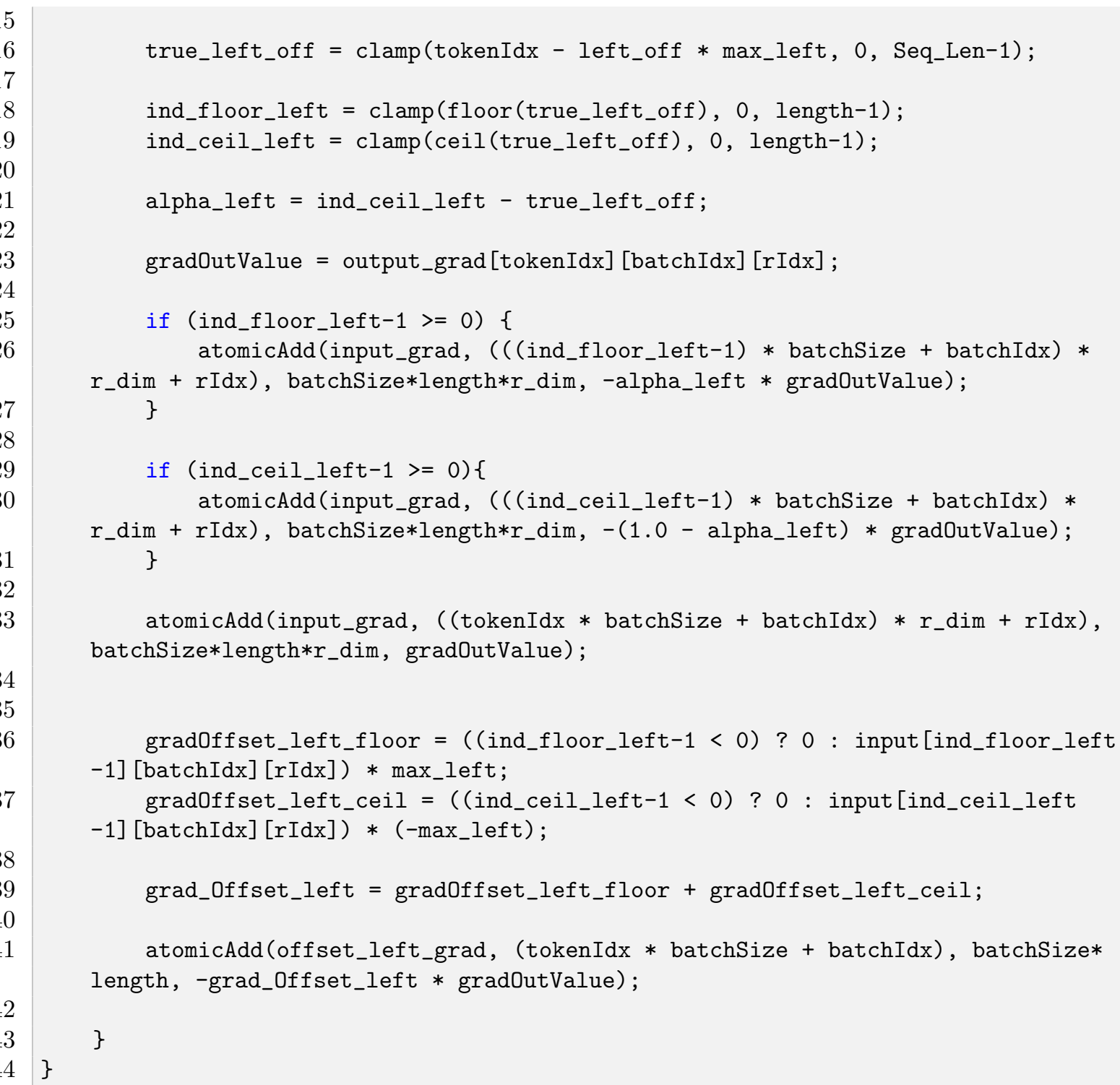

\section{A.3 Pytorch Function Implementation}

Below, we present the python code used for implementing the Pytorch operation for both forward and backward operations. We include both regular and optimized versions of the TaLK Convolution operation. 
